Portland State University

PDXScholar

9-12-1994

\title{
Circuit Modeling of Switched Linear Networks
}

Fardin Ansari

Portland State University

Follow this and additional works at: https://pdxscholar.library.pdx.edu/open_access_etds

Part of the Electrical and Computer Engineering Commons Let us know how access to this document benefits you.

\section{Recommended Citation}

Ansari, Fardin, "Circuit Modeling of Switched Linear Networks" (1994). Dissertations and Theses. Paper 4658.

https://doi.org/10.15760/etd.6542

This Thesis is brought to you for free and open access. It has been accepted for inclusion in Dissertations and Theses by an authorized administrator of PDXScholar. Please contact us if we can make this document more accessible: pdxscholar@pdx.edu. 


\section{THESIS APPROVAL}

The abstract and thesis of Fardin Ansari for the Master of Science in Electrical and Computer Engineering were presented September 12, 1994, and accepted by the thesis committee and the department.

COMMITTEE APPROVALS:

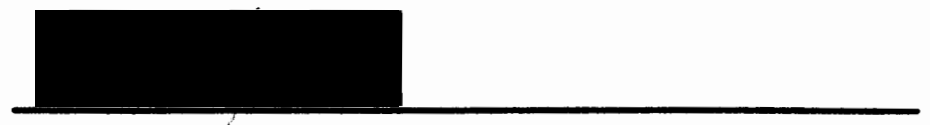

Dr. Richarđ Tymerski, Chair
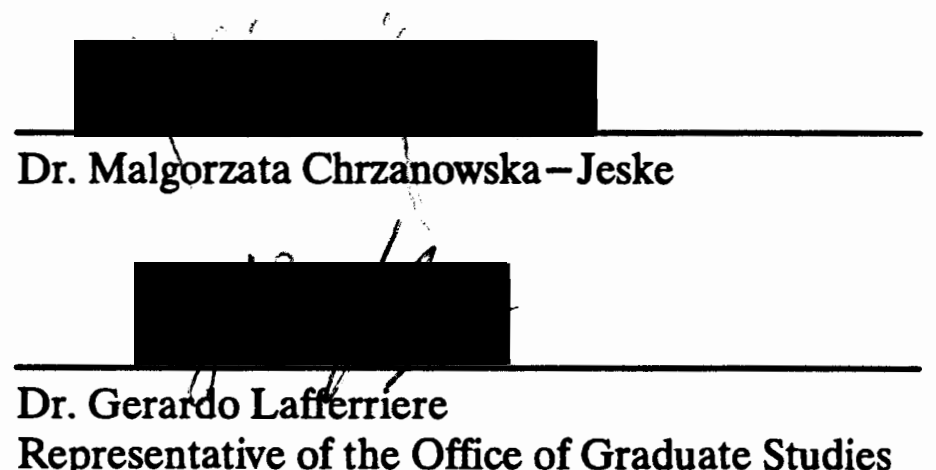

DEPARTMENT APPROVAL:

Dr. Rolf Schaumann, Chair

Department of Electrical Engineering

ACCEPTED FOR PORTLAND STATE UNIVERSITY BY THE LIBRARY

by on 


\begin{abstract}
An abstract of the thesis of Fardin Ansari for the Master of Science in Electrical and Computer Engineering presented September 12, 1994.
\end{abstract}

Title: Circuit Modeling of Switched Linear Networks.

The use of switched linear networks is prevalent in power processing systems such as $d c-t o-d c$ power converters. These converters provide regulated dc output power and are widely used in the power supplies for computers and peripherals.

As with most systems where high performance is a criterion, the power converter is usually enclosed in a feedback loop. Feedback design usually entails the use of small signal (linear) models with linear feedback design techniques.

There exists a large number of small signal models, but most of them are ad hoc. A describing function approach has previously been presented which determines the exact small signal frequency response of the converters. This method is now used to systematically derive circuit models for power converters. The aim of the work presented in this thesis is to model the nonlinear part of the switching converters which are simply the switches. This study is focused on the Pulse Width Modulation (PWM) class of converters. Majority of available models are not very accurate because they are averaged models. The aim of the new approach is to obtain accurate and simple model. A partial realization of this aim has been achieved; the new model is accurate over a very wide frequency range, but presently the parameters are rather complicated. 


\section{CIRCUIT MODELING OF SWITCHED LINEAR NETWORKS}

by

FARDIN ANSARI

A thesis submitted in partial fulfillment of the

requirements for the degree of

MASTER OF SCIENCE

in

\section{ELECTRICAL AND COMPUTER ENGINEERING}

Portland State University

1994 


\section{TABLE OF CONTENTS}

PAGE

ACKNOWLEDGEMENTS ..................... iv

LIST OF FIGURES $\ldots \ldots \ldots \ldots \ldots \ldots \ldots \ldots \ldots \ldots \ldots \ldots \ldots$ v

CHAPTER 1

INTRODUCTION ..........................

\section{CHAPTER 2}

ANALYSIS PRELIMINARIES .................... 6

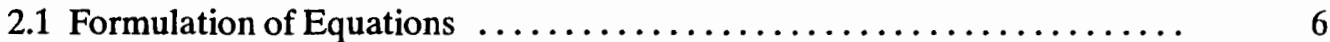

2.1.1 Two Port Networks .................................. 6

2.1.2 State Space Analysis $\ldots \ldots \ldots \ldots \ldots \ldots \ldots \ldots \ldots \ldots \ldots \ldots . \ldots \ldots . \ldots \ldots$

2.1.2.a Low Frequency Approximation $. . \ldots \ldots \ldots \ldots \ldots \ldots \ldots . \ldots$

2.1.2.b Small Signal Approximation .......................... 10

2.1.3 PWM Switch model .................................... 12

2.1.4 Hybrid Parameter Boost Switch Model ....................... 15

2.2 Derivation of Equivalent State Space PWM Boost Model ............. 18

CHAPTER 3

EXACT SMALL SIGNAL ANALYSIS OF PWM

CONVERTERS ........................... 26

3.1 Analysis of Switching Converters in Continuous Conduction Mode ....... 26

3.2 Application of Time Varying Transfer Function ................ 31

3.2.1 Duty Ratio Programmed PWM Converter in CCM .............. 32

3.2.2 Current Programmed Converter in CCM ................. 34

\section{CHAPTER 4}

FIRST MODEL .......................... 37

4.1 PWM Duty Ratio Programmed Model .................... 37

4.2 PWM Current Programmed Model $\ldots \ldots \ldots \ldots \ldots \ldots \ldots \ldots \ldots . \ldots \ldots$

\section{CHAPTER 5}

SECOND MODEL: NEW METHOD FOR OBTAINING

CONTROLLER PARAMETERS

5.1 General Analysis of the New Model ........................ 47

5.1.1 PWM Duty Ratio Programmed Model ..................... 49

5.1 .2 PWM Current Programmed Model ..................... 59

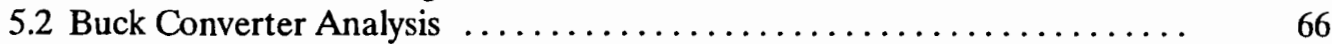

5.2.1 PWM Duty Ratio Programmed Buck Converter ............. 66

5.2.2 PWM Current Programmed Buck Converter ................ 73

5.3 Buckboost Converter Analysis . . . . . . . . . . . . . . . . . . $\quad 80$

5.3.1 PWM Duty Ratio Programmed Buckboost Converter ........... 80

5.3.2 PWM Current Programmed Buckboost Converter ............. 88

CHAPTER 6

THIRD MODEL: NEW METHOD FOR OBTAINING

INPUT PARAMETERS $\ldots \ldots \ldots \ldots \ldots \ldots \ldots \ldots \ldots \ldots \ldots, 93$

6.1 General Analysis of the Model $\ldots \ldots \ldots \ldots \ldots \ldots \ldots \ldots \ldots \ldots \ldots \ldots$ 
6.1.1 PWM Duty Ratio Programmed Model ..................... 98

6.1.2 PWM Current Programmed Model ...................... 105

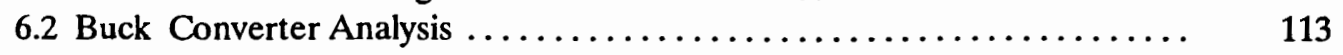

6.2.1 PWM Duty Ratio Programmed Buck Converter .............. 113

6.2.2 PWM Current Programmed Buck Converter ............... 117

6.3 Buckboost Converter Analysis . . . . . . . . . . . . . . . . . . . 120

6.3.1 PWM Duty Ratio Programmed Buckboost Converter ........... 120

6.3.2 PWM Current Programmed Buckboost Converter .............. 124

\section{CHAPTER 7}

CONCLUSION $\ldots \ldots \ldots \ldots \ldots \ldots \ldots \ldots \ldots \ldots \ldots \ldots, 127$

REFERENCES $\ldots \ldots \ldots \ldots \ldots \ldots \ldots \ldots \ldots \ldots \ldots \ldots \ldots, \quad 130$ 


\section{ACKNOWLEDGEMENTS}

First, and foremost, I would like to take this opportunity to express my thanks to my

advisor, Dr. Richard Tymerski, for his guidance throughout this work. It was only based on his theories and previous findings that this thesis could be completed.

I would also like to thank Dr. Malgorzata Chrzanowska-Jeske and Dr. Gerardo Lafferriere for accepting to serve on my committee in their summer break.

I am also thankful to Beth Phelps, for being such an understanding office manager.

I would also like to express my gratitude to staff of the Electrical Engineering department at Portland State University for their help and support all the time.

Many thanks go to my friends around the world. They have all been very supportive throughout the completion of this thesis. Special thanks to Andisheh Sarabi and Stoney Vintson for their helpful comments.

Finally, I wish to express my appreciations to my family in Iran for their patience throughout my stay in the United States. Without their moral support I would not have been able to complete this thesis.

Portland, Oregon

Fardin Ansari

September 1994 


\section{LIST OF FIGURES}

\section{FIGURE}

PAGE

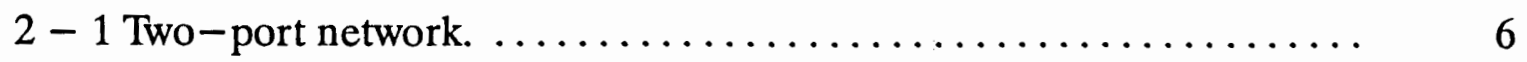

2-2 Equivalent circuit for two-port hybrid model. ............ 7

$2-3$ The three basic PWM converters.

(a) Buck. (b) Boost. (c) Buck-boost. ............. 13

$2-4$ Symbol for PWM Switch. ..................... 13

2-5 Hybrid parameter common passive switch model. ............ 14

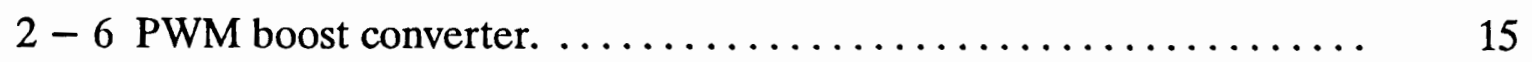

2-7 Small signal hybrid parameter model of the boost converter of Figure $2-6 . \ldots \ldots \ldots \ldots \ldots \ldots \ldots \ldots \ldots \ldots \ldots \ldots \ldots \ldots$

2-8 Boost converter of Figure 2 -6 in; a) DTs subinterval

b) $D^{\prime}$ Ts subinterval. ......................... 18

2 - 9 Hybrid parameter boost model using state space averaging results. .. 23

$2-10$ Typical PWM boost converter. .................... 24

$2-11$ The terminal voltages and currents of the switch implementation of Figure $2-10 . \ldots \ldots \ldots \ldots \ldots \ldots \ldots \ldots \ldots \ldots \ldots \ldots \ldots \ldots \ldots \ldots$

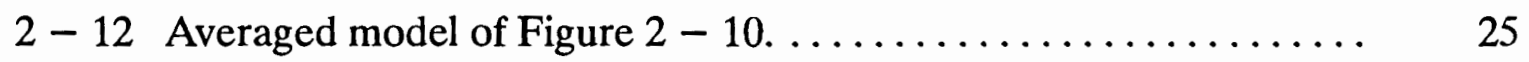

2-13 Small signal equivalent circuit model for the boost converter of Figure $2-10 . \ldots \ldots \ldots \ldots \ldots \ldots \ldots \ldots \ldots \ldots \ldots \ldots \ldots \ldots \ldots \ldots \ldots$

3-1 State and Switching times of the $i-$ th subinterval. ........... 28

$3-2$ Waveforms showing typical signals associated with duty ratio

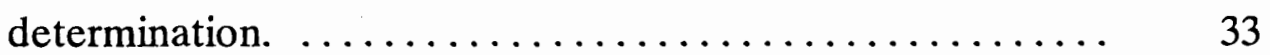

3- 3 Waveforms showing the intersection of voltage ramps that cause switching to take place. ................... 35

4-1 The control-to-output response of the PWM boost converter operating in CCM; exact (solid line) versus the first model

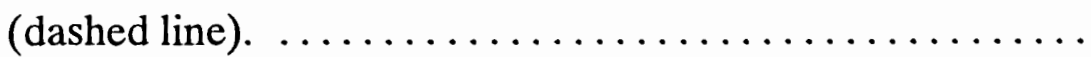

4-2 The input-to-output response of the PWM boost converter operating in CCM; exact (solid line) versus the first model (dashed line). 
4-3 The control-to-output response of the PWM boost converter operating in CCM under current mode programming; exact (solid line) versus the first model (dashed line). .......

4-4 The input-to-output response of the PWM boost converter operating in CCM under current mode programming; exact (solid line) versus the first model (dashed line).

5- 1 The control-to-output response of the PWM boost converter operating in CCM; exact (solid line) versus the second model (dashed line).$\ldots \ldots \ldots \ldots \ldots \ldots \ldots \ldots \ldots \ldots$

$5-2$ The input-to-output response of the PWM boost converter operating in CCM; exact (solid line) versus the second model

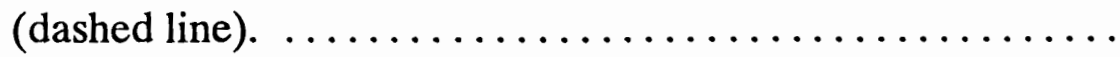

$5-3$ Boost converter: capacitor is replaced by a constant voltage

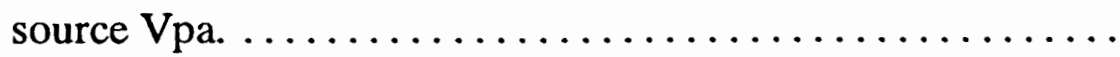

5 - 4 Reduced order boost converter of Figure $5-3$ in; a) DTs subinterval b) D'Ts subinterval.

5 - 5 The control-to-output response of the PWM boost converter operating in CCM; exact (solid line) versus the reduced order second model (dashed line). .................

5-6 The input-to-output response of the PWM boost converter operating in CCM; exact (solid line) versus the reduced second model (dashed line). . . . . . . . . . . . . . .

5 - 7 Hybrid parameter switch model for PWM duty ratio programming. . .

5-8 The control-to-output response of the PWM boost converter operating in CCM under current mode programming; exact (solid line)

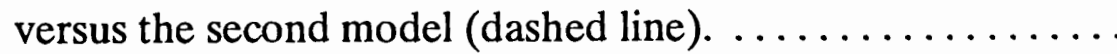

5 - 9 The input-to-output response of the PWM boost converter operating in CCM under current mode programming ; exact (solid line) versus the second model (dashed line) . ............ 6

5 - 10 Hybrid parameter switch model for current mode programming. ...

5- 11 The control-to-output response of the PWM boost converter operating in CCM under current mode programming; exact (solid line) versus the reduced order second model (dashed line). ...... 
5- 12 The input-to-output response of the PWM boost converter operating in CCM under current mode programming; exact (solid line) versus the reduced order second model (dashed line). ..... 65

5 - 13 Circuit schematics for PWM buck converter. .............. 66

5- 14 Hybrid parameter switch model for small-signal analysis of the buck converter of Figure $5-13 . \ldots \ldots \ldots \ldots \ldots \ldots \ldots$

5 - 15 Small-signal control-to-output equivalent model of the buck converter of Figure $5-13 \ldots \ldots \ldots \ldots \ldots \ldots \ldots \ldots \ldots$

5 - 16 Equivalent input-to-ouput small-signal hybrid parameter switch model for the PWM buck converter of Figure 5 - 13...... 69

5- 17 The control-to-output response of the PWM buck converter operating in CCM; exact (solid line) versus the second model

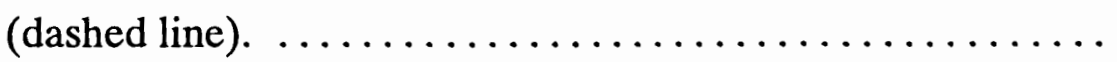

5- 18 The input-to-output response of the PWM buck converter operating in CCM ; exact (solid line) versus the second model (dashed line).

5-19 Circuit schematics for PWM buck converter. ............. 73

5-20 Equivalent small-signal hybrid parameter switch model for the PWM buck converter of Figure $5-19 . \ldots \ldots \ldots \ldots \ldots$

5-21 The control-to-output response of the PWM buck converter operating in CCM under current mode programming; exact (solid line) versus the second model (dashed line). . . . . . . . . . .

5-22 The input-to-output response of the PWM buck converter operating in CCM under current mode programming; exact (solid line) versus the second model (dashed line). . . . . . . . . . $\quad 79$

5 - 23 Circuit schematics for PWM buckboost converter. ............ 80

5-24 Equivalent small-signal hybrid parameter switch model for the PWM buckboost converter of Figure $5-23 . \ldots \ldots \ldots \ldots$

5-25 Equivalent small-signal control-to-output hybrid parameter switch model for PWM buckboost converter of Figure 5-23. ....

5-26 Equivalent input-to-ouput small-signal hybrid parameter switch model for the PWM buckboost converter of Figure $5-23$. . 
5-27 The control-to-output response of the PWM buck-boost converter operating in CCM; exact (solid line) versus the second

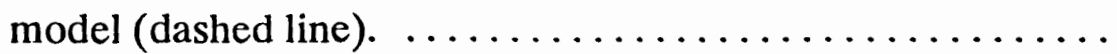

5-28 The input-to-output response of the PWM buck-boost converter operating in CCM; exact (solid line) versus the second model (dashed line).$\ldots \ldots \ldots \ldots \ldots \ldots \ldots \ldots \ldots \ldots \ldots \ldots$

5-29 Circuit schematics for PWM buckboost converter operating in

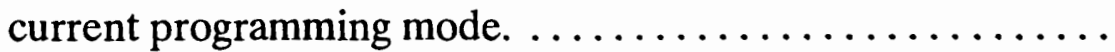

5 - 30 Equivalent small-signal hybrid parameter switch model for current mode analysis of the buckboost converter of Figure $5-23$..

5 - 31 The control-to-output response of the PWM buckboost converter operating in CCM under current mode programming; exact (solid line) versus the second model (dashed line). ....

5 - 32 The input-to-output response of the PWM buckboost converter operating in $\mathrm{CCM}$ under current mode programming; exact (solid line) versus the second model (dashed line). ....

6-1 The control-to-output response of the PWM boost converter operating in CCM; exact (solid line) versus the third model (dashed line). $\quad 99$

6-2 The input-to-output response of the PWM boost converter operating in CCM; exact (solid line) versus the third model (dashed line).

6-3 The hybrid parameter switch model for PWM duty ratio

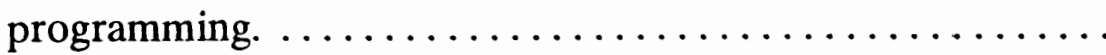

6-4 The control-to-output response of the PWM boost converter operating in CCM; exact (solid line) versus the reduced order third model (dashed line).

6 - 5 The input-to-output response of the PWM boost converter operating in CCM; exact (solid line) versus the reduced third model (dashed line).

6- 6 The control-to-output response of the PWM boost converter operating in CCM under current mode programming; exact (solid line) versus the third model (dashed line) . ................ 
6-7 The input-to-output response of the PWM boost converter operating in CCM under current mode programming ; exact (solid line) versus the third model (dashed line) . ................

6-8 The control-to-output response of the PWM boost converter operating in CCM under current mode programming. Exact (solid line) versus the reduced order third model (dashed line). ...... 108

6 - 9 The input-to-output response of the PWM boost converter operating in CCM under current mode programming. Exact (solid line) versus the reduced order third model (dashed line). . ..... 109

6- 10 Hybrid parameter switch model for current mode programming. ...

6- 11 Hybrid parameter switch model for small-signal analysis of the buck converter of Figure $5-13 \ldots \ldots \ldots \ldots \ldots \ldots \ldots \ldots$

6-12 The control-to-output response of the PWM buck converter operating in CCM; exact (solid line) versus the third model (dashed line).

6 - 13 The input-to-output response of the PWM buck converter operating in CCM ; exact (solid line) versus the third model (dashed line).

6- 14 Hybrid parameter switch model for small-signal analysis of the Current programmed buck converter of Figure 5 - 19. ....

6 - 15 The control to output response of the PWM buck converter operating in CCM under current mode programming; exact (solid line) versus the third model (dashed line) . ..............

6-16 The input-to-output response of the PWM buck converter operating in CCM under current mode programming; exact (solid line) versus the third model (dashed line) . ................

6 - 17 Small - signal hybrid parameter switch model for the PWM duty-ratio buck-boost converter of Figure $5-23 . \ldots \ldots \ldots \ldots \ldots$

6-18 The control-to-output response of the PWM buckboost converter operating in CCM; exact (solid line) versus the third model (dashed line). 
6- 19 The input-to-output response of the PWM buck-boost converter operating in CCM ; exact (solid line) versus the third

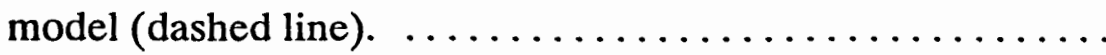

6-20 Small-signal equivalent model for current mode analysis of the buckboost converter in Figure $5-23 . \ldots \ldots \ldots \ldots \ldots \ldots .124$

6 - 21 The control-to-output response of the PWM buckboost converter operating in CCM under current mode programming; exact (solid line) versus the third model (dashed line). ......

6- 22 The input-to-output response of the PWM buck-boost converter operating in CCM under current mode programming; exact (solid line) versus the third model (dashed line). 


\section{CHAPTER 1 INTRODUCTION}

In recent years, small-signal modeling of dynamic behaviors of open-loop $d c-d c$ power converter has received notable amount of attention. This popularity is due to the fact that modeling of the converter by linear circuits is usually the basis by which feedback design is undertaken for designing reliable high performance regulators.

As with most systems, where high performance is a criterion, the power converter is enclosed in a feedback loop. The purpose of the feedback loop is to keep the performance of the system close to the desired operating conditions. In a power converter system, a feedback loop is designed to counteract the disturbances in the line voltage, $v_{g}$, and the load current, $i_{o}$, and subsequently regulate the output voltage, $v_{o}$. These disturbances can be assumed to be of small signal variations. Therefore, a linearized small-signal model is constructed to examine the dynamic behaviors of the converter.

The open loop transfer functions $\hat{v}_{o}(s) / \hat{v}_{g}(s)$ and $\hat{v}_{o}(s) / \hat{i}_{o}(s)$ are derived from the small-signal model and plotted, e.g. Bode, for the frequency response analysis. If necessary, the system is compensated to meet the desired nominal operating conditions.

The importance of small-signal modeling in the design of power stages cannot be overstated. In power electronics, there are many different approaches to small-signal modeling. The majority of models work with only one specific control scheme, e.g. Pulse Width Modulation (PWM) duty-ratio or PWM current programming control. In the duty-ratio control, the switch $\mathrm{ON}$-time is controlled by comparing a sawtooth 
ramp with the controller voltage, $v_{c}$. In the current mode programming, the switch $\mathrm{ON}$-time is determined by comparing a ramp proportional to the inductor current with the control voltage, $v_{c}$. So, in the current mode programming, both the duty ratio and inductor current control the length of the switch ON time. In this section, all the models are separated into three general modeling methods; the exact small-signal analysis techniques [1]-[4], the averaged models [ 5$]-[10]$, and the sampleddata representation models [ 11$]-[12]$.

The easiest and the most widely used method involves averaging. Averaged models are simple to derive but give inaccurate high frequency results. The circuity-parameter can be constructed using the state space averaging approach [ 5], [6]. This model can be used to determine the various impedances and transfer functions of the system. It can also give the designer additional insight into the physical properties of the circuit. Another circuit oriented modeling technique involves the construction of the averaged models for the switch in the converter circuit [ 7]-[10]. This switch is called the PWM switch [ 8 ]. The PWM switch is a single pole double throw switch [ 8 ] responsible for the switching of the converter from one circuit configuration to another during each period. This modeling approach is similar to transistor modeling. The derivation of switch models are simpler than the converter models [9]. The limitation is that the PWM switch arrangement does not appear in all different converter topologies. The averaging techniques are generally used for the PWM duty-ratio programmed analysis.

The small-signal modeling of current mode programming is more complicated. The averaged models $[5]-[10]$ cannot predict the response of the current mode programmed converters. Several attempts have been made to incorporate the current mode programming in the existing averaged models [ 13$]-[16]$. The price has always been complexity or inaccuracy of the augmented model. 
Exact small -signal mathematical models [1]-[4] are very accurate. This method can be applied to any periodic, time-varying, piecewise, linear system. The price for the exactness is the complexity of the matrix manipulations. This modeling technique is automated in a power electronic circuit simulator, named PECS [ 3 ]. PECS can be used to perform analysis of higher order power electronics systems whose complicated behavior cannot be quickly analyzed by hand [ 2 ].

The sampled data models can be derived to obtain accurate response for current mode control [ 11 ]. The basic idea is to form a linear difference equation which describes propagation of a point on a converter waveform from one cycle to another. The analysis of the sampled data models is simpler than the exact small-signal models. However, the sampling theorem applies to the sampled data models which restricts the upper limit of the frequency range to half the switching frequency.

In general, the majority of models work with only one specific control scheme (be it the duty ratio [ 6 ], current mode control [ 11 ], etc.). From the above, it can be inferred that there is a need for a unified model applicable to various regulating schemes.

It is the purpose of this thesis to present a unified small-signal modeling approach applicable to any power converter system representable by two-port networks. This method can be used to represent the converter, the switch, or other three terminal components in the circuit by a two-port network and obtain the linearized small-signal model. Modeling the PWM switch is less complicated than modeling the whole circuit; therefore, in this thesis, the nonlinear PWM switch is replaced by its linearized small signal two-port model. The expressions for the model parameters are obtained using the small-signal describing functions [ 1$],[3]$. This model is very accurate up to the switching frequency. The new modeling approach can be used with any set of transfer functions such as state-space transfer functions to obtain expressions for the parameters. 
In chapter 2, a review of hybrid parameter two-port model, state space averaging analysis, the PWM switch model, and an introduction to the new modeling approach is given. In section 2.1, the three terminal PWM switch is replaced by an augmented two-port hybrid parameter model. The proposed modeling approach is first used to obtain the state-space averaging model by applying it to a boost converter. A boost converter is one of the three basic converter types with PWM switch implementation. Using the new approach the PWM switch in the boost converter is replaced by a hybrid parameter switch model. Then, the hybrid parameters are evaluated using state-space control-to-output and input-to-output transfer functions. Next, the resulting parameter expressions are compared with the expressions obtained through the circuit averaging technique.

In chapter 3 , a review of exact analysis of switching converters is presented followed by a section on the application of the time varying transfer function. In the latter, the exact small-signal analysis for PWM duty ratio programmed converters and PWM current programmed converters operating in continuous conduction mode (CCM) are presented.

In chapter 4, numeric expressions for the switch parameters are obtained using exact small-signal describing functions. First, the switch parameters for the boost converter are analyzed. Then, the control-to-output, $\hat{v}_{o}(s) / \hat{v}_{c}(s)$, and the input-to-output, $\hat{v}_{o}(s) / \hat{v}_{g}(s)$ responses of the model are plotted against the exact results. The control-to-output transfer function is part of the loop gain; therefore, its response is important for stability analysis of the regulator. The input-to-output response is however, considered less important when designing the regulator. This model fails to predict exact control-to-output frequency response. Therefore, a second, improved approach is proposed in chapter 5 , which can produce exact control-to-output results. In order to obtain simple parameter expressions, the output capacitor is replaced 
by a constant voltage source. This assumption reduces the number of states to one, namely the inductor current state, $i_{L}$. The reduced order model is then shown to be inaccurate for the input - to-output response analysis by testing the model on the buck and buckboost converters. The reason for this inaccuracy is discussed in chapter 6 , where the third approach is presented. The small-signal control-to-output, and input-to-output frequency response of this model are compared with those of the exact transfer functions. It will be seen that the this model predicts the exact results at least up to the switching frequency. Next, the third model is used to analyze the buck and buckboost converters. The resulting plots show an excellent agreement with the exact small-signal control - to-output and input-to-output responses. The third modeling approach holds valid for both PWM duty-ratio and PWM current mode programming.

The price for the exactness of the third model is, however, the complexity of the parameter expressions. This model is an excellent choice for computer implementation. The current programmed model can be used to obtain the PWM duty ratio model by setting a parameter to zero. State space transfer functions can be used with this model which enables the designers to perform stability analysis, i.e. determine transfer functions, poles and zeros of the system. 


\section{CHAPTER 2}

\section{ANALYSIS PRELIMINARIES}

\subsection{Formulation of Equations}

In this section, a unified small signal switch model based on two-port network analysis is proposed. A review of hybrid parameters, state space averaging analysis, and the PWM switch model will be given first. Next, the new hybrid parameter switch model will be introduced using state space averaging transfer functions. In the following chapters, this model is further developed using exact small signal transfer functions proposed in [1] and later extended in [3].

\subsubsection{Two Port Networks}

It is a common practice in circuit analysis to model three terminal devices in terms of their terminal characteristics using two-port networks. A two-port network is composed of linear elements such as $R, L, C$, and dependent sources. It contains no independent sources except at the ports. Figure 2-1 sketches the two-port network.

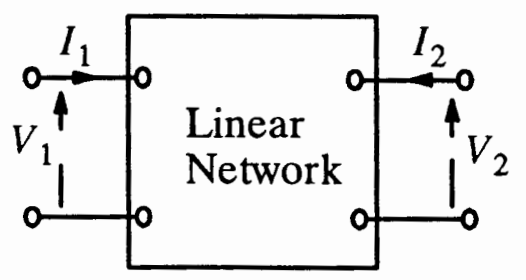

Figure 2-1 Two-port network. 
Taking $I_{1}$ and $V_{2}$ to be the excitation variables (independent sources), it is possible to determine the other two response variables (dependent sources) through parameters known as hybrid parameters. As the network is assumed to be linear, it is possible to utilize the principle of superposition. By application of this principle, the values for the variables $V_{1}$ and $I_{2}$ can be determined. $V_{1}$ consists of two parts, one due to the input current $\left(I_{1}\right)$ and another due to the output voltage $\left(V_{2}\right) . \quad I_{2}$ can be found in the same manner.

In general, two-port equations using the hybrid parameters are;

$$
\begin{aligned}
& V_{1}=h_{11} I_{1}+h_{12} V_{2} \\
& I_{2}=h_{21} I_{1}+h_{22} V_{2}
\end{aligned}
$$

The hybrid equivalent circuit model representing equations $(2-1)$ and $(2-2)$ is shown in Figure $2-2$.
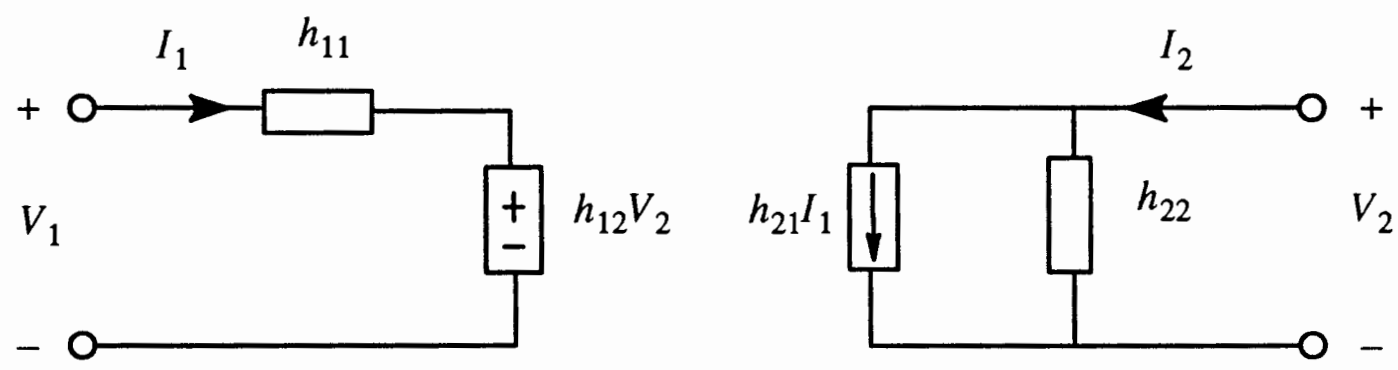

Figure 2 - 2 Equivalent circuit for two-port hybrid model. 
The hybrid parameters are determined by the following equations:

$$
\begin{aligned}
& h_{11}=\frac{V_{1}}{I_{1}} \mid V_{2}=0 \\
& h_{12}=\frac{V_{1}}{V_{2}} \mid I_{1}=0 \\
& h_{21}=\frac{I_{2}}{I_{1}} \mid V_{2}=0 \\
& h_{22}=\frac{I_{2}}{V_{2}} \mid I_{1}=0
\end{aligned}
$$

where

$h_{11}$ is the short circuit input impedance, $h_{12}$ is the open-circuit reverse voltage gain, $h_{21}$ is the short-circuit forward current gain, and $h_{22}$ is the open-circuit output admittance.

\subsubsection{State Space Analysis}

Switching converters are nonlinear systems which can be described by the following general state and output equation:

$$
\begin{aligned}
& \dot{x}(t)=A(t) x(t)+B(t) u(t) \\
& y(t)=C(t) x(t)+E(t) u(t)
\end{aligned}
$$

where $\{A(t), B(t), C(t), E(t)\}$ are the state, the input, the output, and the transmission matrices, respectively. The system is controlled by the complementary switching functions $q(t)$ and $q^{\prime}(t)$ as shown below:

$$
\begin{aligned}
& A(t)=A_{1} q(t)+A_{2} q^{\prime}(t) \\
& B(t)=B_{1} q(t)+B_{2} q^{\prime}(t) \\
& C(t)=C_{1} q(t)+C_{2} q^{\prime}(t)
\end{aligned}
$$




$$
E(t)=E_{1} q(t)+E_{2} q^{\prime}(t)
$$

where $\left\{A_{1}, B_{1}, C_{1}, E_{1}\right\}$ and $\left\{A_{2}, B_{2}, C_{2}, E_{2}\right\}$ consist of linear electrical elements.

Since the converter switches between two subintervals then equations $(2-4)$ may not be continuous over the entire switching period. This can be explained in terms of the energy storing elements such as capacitors and inductors in the converter. Inductor current , capacitor voltage, and their derivatives appearing as state variables in equations $(2-4)$ must be continuous in time in order for the system to be considered continuous. In the conveter circuits, the inductor current waveform is continuous, but its derivative which is the voltage across the inductor is discontinuos. Therefore, to perform circuit analysis techniques the discontinuous nonlinear system must be approximated to continuous linear system.

State space averaging is used to represent switching converters as linear continuous systems. In the sequel, two approximations have been used; 1) low frequency approximation 2) small-signal approximation.

\subsection{2.a Low Frequency Approximation}

As explained earlier, equations $(2-4)$ and $(2-5)$ are difficult to analyze over the entire frequency range. The main reason for this is the discontinuity of the switching times, $q(t)$ and $q^{\prime}(t)$. Therefore, using low frequency approximation, $q(t)$ and $q^{\prime}(t)$ are replaced by their low frequency components, $d$ and $d^{\prime}$, respectively. Hence,

$$
\begin{aligned}
& A=A_{1} d+A_{2} d^{\prime} \\
& B=B_{1} d+B_{2} d^{\prime} \\
& C=C_{1} d+C_{2} d^{\prime} \\
& E=E_{1} d+E_{2} d^{\prime}
\end{aligned}
$$

The quadruples $\{A, B, C, E\}$ are functions of time. Equations $(2-4)$ and $(2-5)$ 
can be rewritten in the following form:

$$
\begin{aligned}
& \dot{x}=A x+B u \\
& y=C x+E u
\end{aligned}
$$

The validity of this approximation is based on the assumption that natural frequencies of the converter, regulator, and line variations are at least one decade lower than the switching frequency.

\subsection{2.b Small Signal Approximation}

Using state space averaging, equations $(2-4)$ and $(2-5)$ were replaced by continuous averaged equations $(2-14)$ and $(2-15)$. Now, the averaged equations are still nonlinear and need to be linearized. Therefore, all the variables in equations $(2-14)$ and $(2-15)$ are replaced by their steady state, and a perturbation term.

Perturbation in the duty ratio will result in

$$
d=D+\hat{d}
$$

and,

$$
d^{\prime}=1-d=1-D-\hat{d}=D^{\prime}-\hat{d}
$$

where $\mathrm{D}$ is the steady state value of the duty ratio and, $\hat{d}$ is the perturbed duty ratio quantity.

Perturbing $(2-14)$ and $(2-15)$ gives:

$$
\begin{aligned}
& \dot{X}+\dot{\hat{x}}=A(X+\hat{x})+B(U+\hat{u}) \\
& Y+\hat{y}=C(X+\hat{x})+E(U+\hat{u})
\end{aligned}
$$

where $d$ in $A, B, C$, and $E$ is also perturbed as shown below:

$$
\begin{aligned}
& A=A_{1}(D+\hat{d})+A_{2}\left(D^{\prime}-\hat{d}\right) \\
& B=B_{1}(D+\hat{d})+B_{2}\left(D^{\prime}-\hat{d}\right)
\end{aligned}
$$




$$
\begin{aligned}
& C=C_{1}(D+\hat{d})+C_{2}\left(D^{\prime}-\hat{d}\right) \\
& E=E_{1}(D+\hat{d})+E_{2}\left(D^{\prime}-\hat{d}\right)
\end{aligned}
$$

Substituting equations $(2-20)$ and $(2-21)$ into equation $(2-18)$ gives:

$$
\begin{aligned}
\dot{X}+\dot{\hat{x}}=\left[A_{1}(\right. & \left.D+\hat{d})+A_{2}\left(D^{\prime}-\hat{d}\right)\right](X+\hat{x}) \\
+ & {\left[B_{1}(D+\hat{d})+B_{2}\left(D^{\prime}-\hat{d}\right)\right](U+\hat{u}) }
\end{aligned}
$$

Separating all the ac perturbation terms from $(2-24)$ one gets:

$$
\begin{aligned}
\dot{\hat{x}}=\left[A_{1}(D+\hat{d})+A_{2}\left(D^{\prime}-\hat{d}\right)\right] \hat{x}+\left(A_{1} \hat{d}-A_{2} \hat{d}\right) X \\
+\left[B_{1}(D+\hat{d})+B_{2}\left(D^{\prime}-\hat{d}\right)\right] \hat{u}+\left(B_{1} \hat{d}-B_{2} \hat{d}\right) X
\end{aligned}
$$

which simplifies to:

$$
\begin{aligned}
\dot{\hat{x}}=\left(A_{1} D\right. & \left.+A_{2} D^{\prime}\right) \hat{x}+\left(A_{1}-A_{2}\right)(X \hat{d})+\left(A_{1}-A_{2}\right)(\hat{x} \hat{d}) \\
& +\left(B_{1} D+B_{2} D^{\prime}\right) \hat{u}+\left(B_{1}-B_{2}\right)(U \hat{d})+\left(B_{1}-B_{2}\right)(\hat{u} \hat{d})
\end{aligned}
$$

Equations $(2-26)$ contains nonlinear terms which are products of two perturbation terms.

Similarly, the output equation $(2-19)$ can also be expanded using equations $(2-22)$ and $(2-23)$ to give the following ac result:

$$
\begin{aligned}
\hat{y}=\left(C_{1} D\right. & \left.+C_{2} D^{\prime}\right) \hat{x}+\left(C_{1}-C_{2}\right)(X \hat{d})+\left(C_{1}-C_{2}\right)(\hat{x} \hat{d}) \\
& +\left(E_{1} D+E_{2} D^{\prime}\right) \hat{u}+\left(E_{1}-E_{2}\right)(U \hat{d})+\left(E_{1}-E_{2}\right)(\hat{u} \hat{d})
\end{aligned}
$$

To remove the nonlinear terms from equations $(2-26)$ and $(2-27)$, the ac perturbations are assumed to be very small compared to the steady state operating values:

$$
\begin{aligned}
& \|\hat{x}\| \ll\|X\| \\
& \|\hat{d}\| \ll\|D\| \\
& \|\hat{u}\| \ll\|U\|
\end{aligned}
$$


$\|\hat{y}\| \ll\|Y\|$

where $\|$.l denotes a matrix norm.

Dropping the product of ac terms in equations $(2-26)$ and $(2-27)$ and rearranging gives the following ac small signal result:

$$
\begin{aligned}
& \dot{\hat{x}}=A \hat{x}+B \hat{u}+B_{d} \hat{d} \\
& y=C \hat{x}+E \hat{u}+E_{d} \hat{d} \\
& A=D A_{1}+D^{\prime} A_{2} \\
& B=D B_{1}+D^{\prime} B_{2} \\
& C=D C_{1}+D^{\prime} C_{2} \\
& E=D E_{1}+D^{\prime} E_{2} \\
& B_{d}=\left(A_{1}-A_{2}\right) X+\left(B_{1}-B_{2}\right) U \\
& E_{d}=\left(C_{1}-C_{2}\right) X+\left(E_{1}-E_{2}\right) U
\end{aligned}
$$

The small-signal state space frequency response of an ideal dc-to-dc converter described by equation $(2-29)$ is given by equation $(2-36)$.

$$
\hat{y}(j \omega)=\left(C(j \omega I-A)^{-1} B+E\right) \hat{u}(j \omega)+\left(C(j \omega I-A)^{-1} B_{d}+E_{d}\right) \hat{d}(j \omega)
$$

\subsubsection{PWM Switch model}

The basic PWM converters are the buck, boost, and buckboost which are shown in Figure 2-3. 


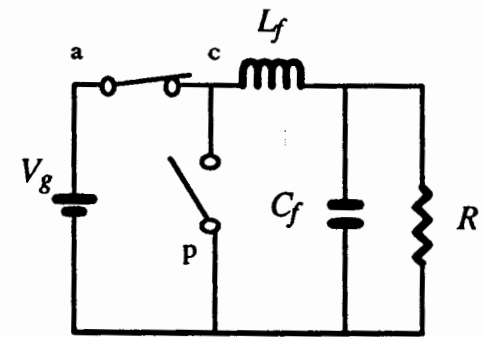

(a)

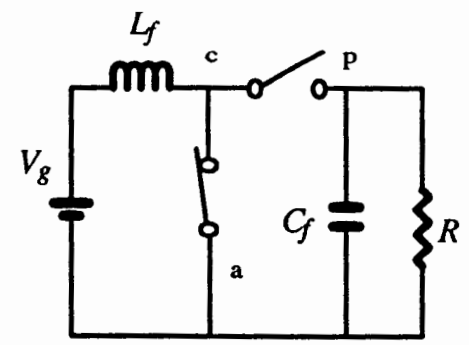

(b)

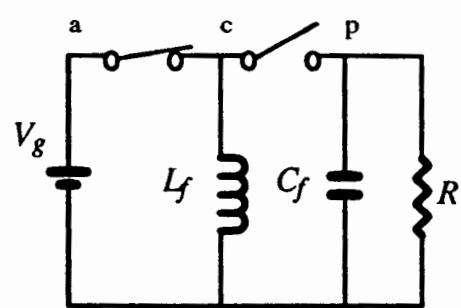

(c)

Figure 2- 3 The three basic PWM converters. (a) Buck. (b) Boost. (c) Buck-boost.

The switch in these converters can be represented as a single-pole double-throw (SPDT) switch called the PWM switch. This implementation satisfies the condition that the switch pairs cannot both be ON or OFF at the same time. A PWM switch is a three-terminal device that may be depicted as in Figure $2-4$, where a, p, and c refer to active, passive and common terminals. The active-common switch is usually a transistor, and the passive-common is a diode.

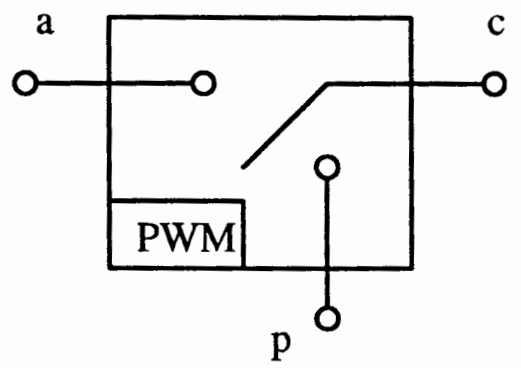

Figure 2 - 4 Symbol for PWM Switch.

The PWM switch can be modeled as a two-port network. The output of each port in the low frequency small signal sense is described by the frequency response relationship given in equation $(2-36)$. The hybrid parameter model in Figure $2-2$ can be augmented to incorporate the additional controller input terms as shown in Figure 2-5. 


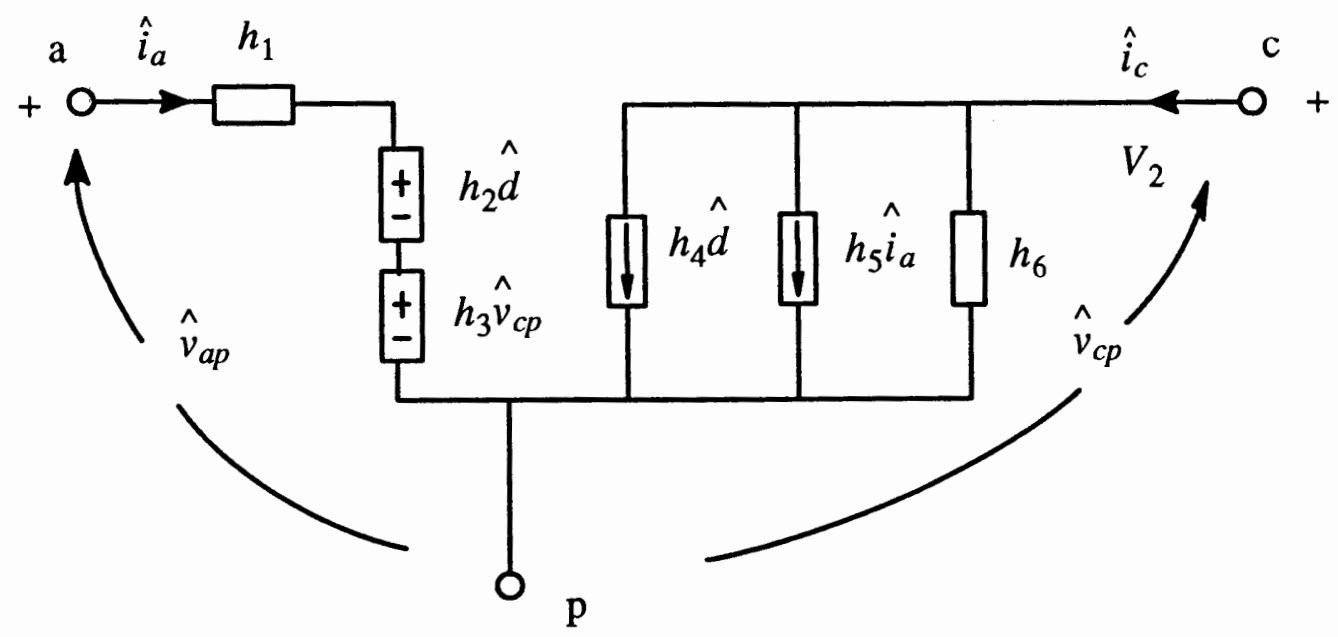

Figure 2 - 5 Hybrid parameter common passive switch model.

Mathematical description of Figure $2-5$ is given below.

$$
\begin{aligned}
& \hat{v}_{a p}=h_{1} \hat{i}_{a}+h_{2} \hat{d}+h_{3} \hat{v}_{c p} \\
& \hat{i_{c}}=h_{4} \hat{i}_{a}+h_{5} \hat{d}+h_{6} \hat{v}_{c p}
\end{aligned}
$$

Each port described by equations $(2-37)$ and $(2-38)$ has one output and three inputs. Furthermore, equations $(2-37)$ and $(2-38)$ can be expressed in terms of the small signal frequency response given in equation $(2-36)$.

$$
\begin{array}{r}
\hat{v}_{a p}(j \omega)=\left(C(j \omega I-A)^{-1} B+E\right) \hat{i}_{a}(j \omega)+\left(C(j \omega I-A)^{-1} B_{d}+E_{d}\right) \hat{d}(j \omega) \\
+\left(C(j \omega I-A)^{-1} B+E\right) \hat{v}_{c p}(j \omega) \\
\begin{aligned}
\hat{i}_{c}(j \omega)=\left(C(j \omega I-A)^{-1} B+E\right) \hat{i}_{a}(j \omega)+\left(C(j \omega I-A)^{-1} B_{d}+E_{d}\right) \hat{d}(j \omega) \\
+\left(C(j \omega I-A)^{-1} B+E\right) \hat{v}_{c p}(j \omega)
\end{aligned}
\end{array}
$$


To find the transfer function from one of the inputs to the output, the other two inputs must be set to zero. The model depicted in Figure $2-5$ is a common passive hybrid parameter switch model. Other switch models can be found using the same technique.

In the following section, the hybrid parameter switch model for the PWM boost converter will be introduced. The switch in the boost converter is in the common active configuration as shown in Figure $2-3(\mathrm{~b})$. In the chapters to follow, this switch model will be used to construct small signal equivalent circuits for other converters.

\subsubsection{Hybrid Parameter Boost Switch Model}

In this section, the parameters for the PWM boost converter switch model will be introduced. The switch configuration of the boost converter of Figure $2-6$ will be represented by the hybrid parameter switch model shown in Figure $2-7$.

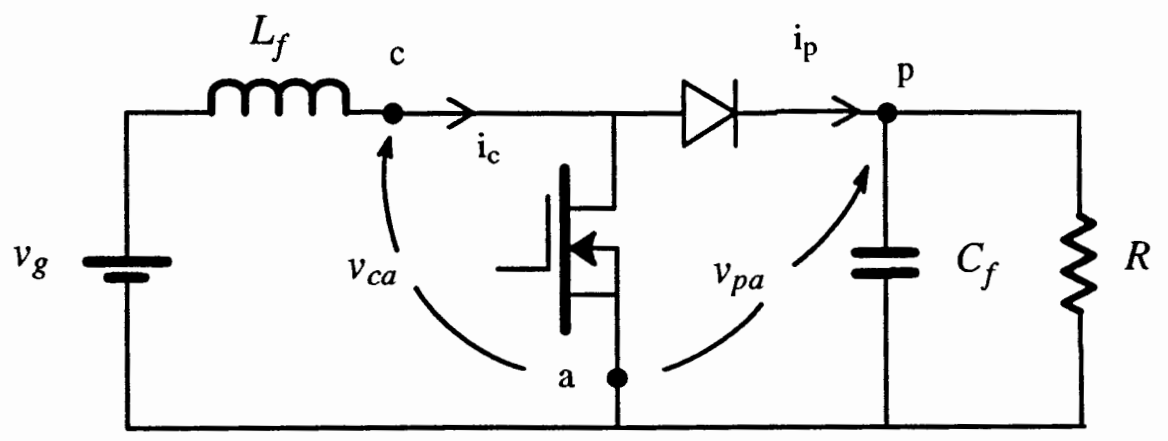

Figure 2-6 PWM boost converter. 


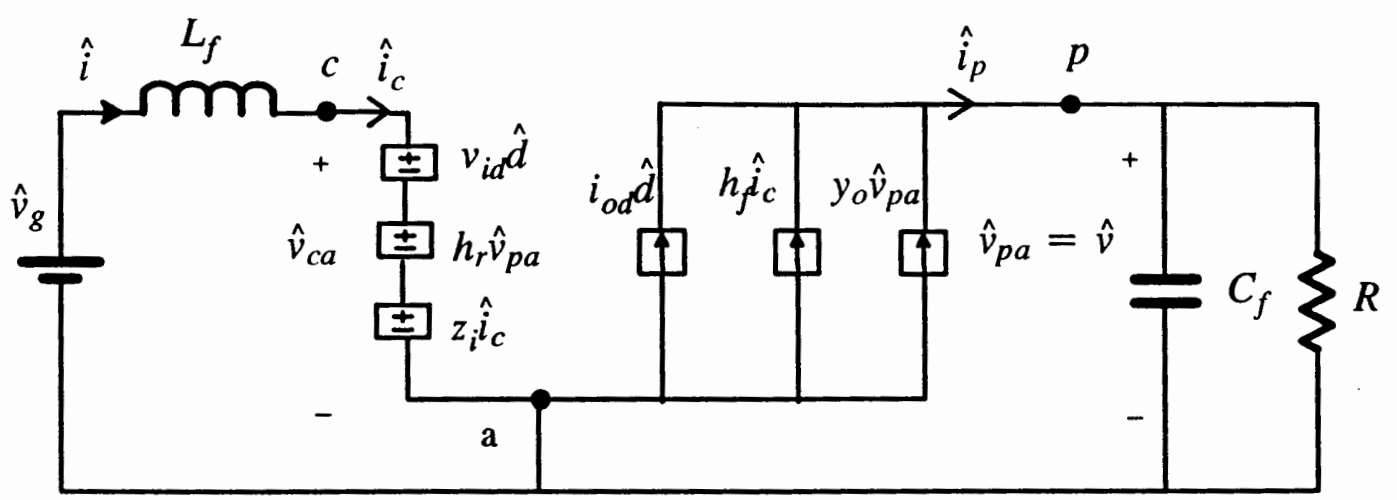

Figure 2- 7 Small signal hybrid parameter model of the boost converter of Figure $2-6$.

In Figure $2-7$, the input port equation is

$$
\hat{v}_{c a}=v_{i d} \hat{d}+h_{r} \hat{v}_{p a}+z_{i} \hat{i}_{c}
$$

and the output port equation is

$$
\hat{i_{p}}=i_{o d} \hat{d}+h_{f_{c}}+y_{o} \hat{v}_{p a}
$$

The above set of equations describes the common active hybrid parameter switch model. The hybrid parameters are obtained by the superposition property of linear networks. In other words, each parameter is found by deactivating the effect of the other two sources in the port equation as shown below:

$$
\begin{aligned}
& v_{i d}=\frac{\hat{v_{c a}}}{\hat{d}}\left|\begin{array}{ll}
\hat{v}_{p a}=0 \\
\hat{i_{c}=0}
\end{array} \quad(2-43) \quad i_{\text {od }}=\frac{\hat{i_{p}}}{\hat{\hat{d}}}\right| \begin{array}{c}
\hat{v}_{p a}=0 \\
\hat{i_{c}}=0
\end{array} \quad(2-44) \\
& h_{r}=\frac{\hat{v}_{c a}}{\hat{v}_{p a}}\left|\begin{array}{l}
\hat{d}=0 \\
\hat{i_{c}}=0
\end{array} \quad(2-45) \quad h_{f}=\frac{\hat{i_{p}}}{\hat{\lambda}}\right| \begin{array}{c}
\hat{d}=0 \\
\hat{i}_{c a}=0
\end{array} \quad(2-46)
\end{aligned}
$$

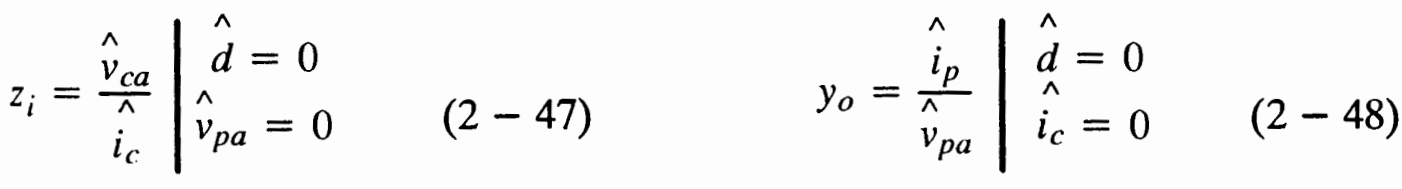


Next, the state-space realization of the small-signal transfer functions has been used to obtain a state-space averaged model and the result has been verified using the circuit averaging method. The state-space analysis is performed to show the simplicity of the new method in obtaining equivalent circuit models. 


\subsection{Derivation of Equivalent State Space PWM Boost Model}

In this section, the new hybrid parameter switch model is used to obtain a small signal state-space averaging model. Next, the same model is found using circuit averaging techniques. It is the purpose of this section to show, in its simplest form, how this modeling approach works.

An analytical expression for each parameter of equations $(2-41)$ and $(2-42)$ can be found by evaluating the appropriate state space transfer functions given in $(2-49)$ and $(2-50)$.

$$
\begin{aligned}
& \frac{\hat{y}}{\hat{u}}(j \omega)=C(j \omega I-A)^{-1} B+E \\
& \frac{\hat{y}}{\hat{\lambda}}(j \omega)=C(j \omega I-A)^{-1} B_{d}+E_{d}
\end{aligned}
$$

Consider the boost converter of Figure $2-6$. For this converter to operate in continuous conduction mode, the state equations have to be obtained for both $D T_{s}$ and $D^{\prime} T_{s}$ subintervals. As shown in Figure $2-8$, it is possible to find state and output matrices for each subinterval.

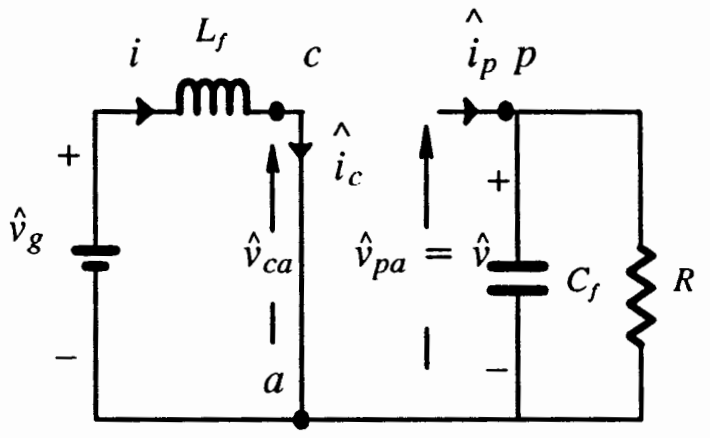

(a)

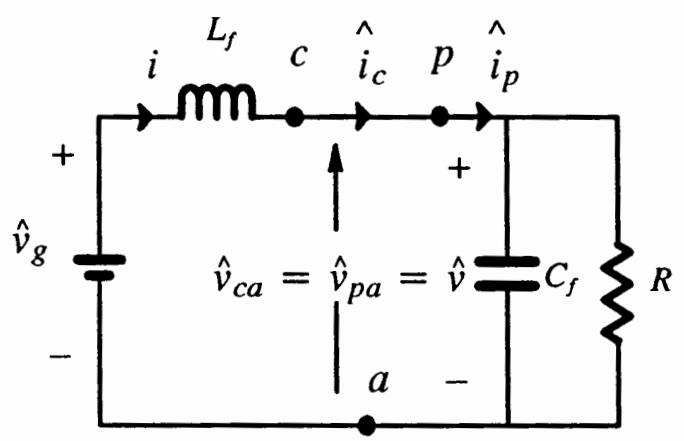

(b)

Figure $2-8$ Boost converter of Figure $2-6 \mathrm{in}$; a) $D T_{s}$ subinterval b) $D^{\prime} T_{s}$ subinterval. 
State equations are dependent solely on the states $x=\left[\begin{array}{ll}i_{c} & v_{p a}\end{array}\right]^{T}$ and input $u=V g$ of the system. Each subinterval is described by a set of state equations.

The behavior of the converter in $D T_{s}$ is described by

$$
\dot{x}=A_{1} x+B_{1} u
$$

and the state matrices for this subinterval are

$$
A_{1}=\left[\begin{array}{cc}
0 & 0 \\
0 & -1 / R C_{f}
\end{array}\right]
$$

and

$$
B_{1}=\left[\begin{array}{c}
\frac{1}{L_{f}} \\
0
\end{array}\right]
$$

Similarly the behavior of the converter in $D^{\prime} T_{s}$ is described by

$$
\dot{x}=A_{2} x+B_{2} u
$$

where

$$
A_{2}=\left[\begin{array}{cc}
0 & -1 / L_{f} \\
1 / C_{f} & -1 / R C_{f}
\end{array}\right]
$$

and

$$
B_{2}=\left[\begin{array}{c}
\frac{1}{L_{f}} \\
0
\end{array}\right]
$$

Using equations $(2-30),(2-31)$, and $(2-34)$ one has:

$$
A=\left[\begin{array}{cc}
0 & -\frac{D^{\prime}}{L_{f}} \\
\frac{D^{\prime}}{C_{f}} & -\frac{1}{R C_{f}}
\end{array}\right] \quad B=\left[\begin{array}{c}
\frac{1}{L_{f}} \\
0
\end{array}\right] \quad B_{d}=\left[\begin{array}{c}
\frac{V_{p a}}{L_{f}} \\
-\frac{I_{c}}{C_{f}}
\end{array}\right]
$$


The $C$ and $E$ matrices are obtained using equations $(2-32)$ and $(2-33)$, respectively:

When $y=\hat{v}_{c a}$ :

$$
\begin{aligned}
& C_{1}=\left[\begin{array}{ll}
0 & 0
\end{array}\right] \quad E_{1}=0 \\
& C_{2}=\left[\begin{array}{ll}
0 & 1
\end{array}\right] \quad E_{2}=0 \\
& C=\left[\begin{array}{ll}
0 & D^{\prime}
\end{array}\right] \quad \text { and } \quad E=0
\end{aligned}
$$

When $y=\hat{i}_{p}$ :

$$
\begin{aligned}
& C_{1}=\left[\begin{array}{ll}
0 & 0
\end{array}\right] \quad E_{1}=0 \\
& C_{2}=\left[\begin{array}{ll}
1 & 0
\end{array}\right] \quad E_{2}=0 \\
& C=\left[\begin{array}{ll}
D^{\prime} & 0
\end{array}\right] \quad \text { and } \quad E=0
\end{aligned}
$$

When $y=\hat{i}_{c}$ :

$$
\begin{aligned}
& C_{1}=\left[\begin{array}{ll}
1 & 0
\end{array}\right] \quad E_{1}=0 \\
& C_{2}=\left[\begin{array}{ll}
1 & 0
\end{array}\right] \quad E_{2}=0 \\
& C=\left[\begin{array}{ll}
1 & 0
\end{array}\right] \quad \text { and } \quad E=0
\end{aligned}
$$

When $y=\hat{v}_{p a}$ :

$$
\begin{aligned}
& C_{1}=\left[\begin{array}{ll}
0 & 1
\end{array}\right] \quad E_{1}=0 \\
& C_{2}=\left[\begin{array}{ll}
0 & 1
\end{array}\right] \quad E_{2}=0 \\
& C=\left[\begin{array}{ll}
0 & 1
\end{array}\right] \quad \text { and } \quad E=0
\end{aligned}
$$

From now deactivating the effect of particular terms of an equation will be referred to as nulling. As evident from equations $(2-41)$ and $(2-42)$, two of the independent sources of the hybrid parameter model are the states of the system; therefore, any of the states can be nulled by setting the corresponding column of the $C$ vector to zero. 
First, the control parameter $v_{i d}$ can be solved for:

$v_{i d}=\frac{\hat{v}_{c a}}{\hat{d}} \mid \begin{aligned} & \hat{v}_{p a}=0 \\ & \hat{i_{c}}=0\end{aligned}$

Using $(2-50)$ one has

$v_{i d}=C(j \omega I-A)^{-1} B_{d}+E_{d}$

where

$E_{d}=\left(C_{1}-C_{2}\right) X+\left(E_{1}-E_{2}\right) U$

As a constraint imposed on equation $(2-43)$, both states of equation $(2-50)$ need to be nulled. Therefore, $\mathrm{C}$ in equation $(2-53)$ will be forced to give:

$$
C=\left[\begin{array}{ll}
0 & 0
\end{array}\right]
$$

and consequently

$$
v_{i d}=E_{d}=-V_{p a}
$$

Next an expression for the $i_{o d}$ parameter will be found using equation $(2-44)$. Nulling both states of the corresponding $C$ matrix will reduce equation $(2-50)$ to :

$$
i_{o d}=E_{d}=-I_{c}
$$

In general, nulling both states of equation $(2-50)$ will give:

$$
\frac{\hat{y}}{\hat{d}}(j \omega)=E_{d}
$$

Solving for the $h_{r}$ parameter of equation $(2-45)$ is tricky. The $h_{r}$ parameter is an output-to-output transfer function which cannot be solved unless it is put in a standard input - to-output form. This is done by dividing both numerator and denominator of equation $(2-45)$ by the input $\hat{v}_{g}$. Equation $(2-49)$ can now be used to obtain 
an expression for the $h_{r}$ parameter.

$$
h_{r}=\frac{\hat{v}_{c a} / \hat{v}_{g} \mid \begin{array}{l}
\hat{d}=0 \\
\hat{i_{c}}=0
\end{array}}{\hat{v}_{p a} / \hat{v}_{g} \mid \begin{array}{l}
\hat{d}=0 \\
\hat{i}_{c}=0
\end{array}}
$$

Since $E$ equals zero for the numerator as well as the denominator of equation $(2-45)$, then equation $(2-49)$ reduces to:

$$
h_{r}=C(j \omega I-A)^{-1} B
$$

The numerator of the $h_{r}$ is :

$$
\hat{v}_{c a} / \hat{v}_{g} \mid \begin{aligned}
& \hat{d}=0 \\
& \hat{i_{c}}=0
\end{aligned}=\left[\begin{array}{ll}
0 & D^{\prime}
\end{array}\right] \frac{1}{\Delta}\left[\begin{array}{cc}
s+\frac{1}{R C_{f}} & -\frac{D^{\prime}}{L_{f}} \\
\frac{D^{\prime}}{C_{f}} & s
\end{array}\right]\left[\begin{array}{c}
\frac{1}{L_{f}} \\
0
\end{array}\right]=\frac{1}{\Delta} \frac{D^{\prime 2}}{L_{f} C_{f}}
$$

and the denominator is:

$$
\hat{v}_{p a} / \hat{v}_{g} \mid \begin{aligned}
& \hat{d}=0 \\
& \hat{i}_{c}=0
\end{aligned}=\left[\begin{array}{ll}
0 & 1
\end{array}\right] \frac{1}{\Delta}\left[\begin{array}{cc}
s+\frac{1}{R C_{f}} & -\frac{D^{\prime}}{L_{f}} \\
\frac{D^{\prime}}{C_{f}} & s
\end{array}\right]\left[\begin{array}{c}
\frac{1}{L_{f}} \\
0
\end{array}\right]=\frac{1}{\Delta} \frac{D^{\prime}}{L_{f} C_{f}}
$$

Therefore, the overall result is:

$$
h_{r}=\frac{D^{\prime 2} / L_{f} C_{f}}{D^{\prime} / L_{f} C_{f}}=D^{\prime}
$$

As it is evident from $C$ matrices of equations $(2-62)$ and $(2-63)$, the effect of the $\hat{i}_{c}$ state is already zero. This could be explained better using the time domain output equation $(2-29)$ :

$$
y=\hat{C x}+E \hat{u}+E_{d} \hat{d}
$$


First, the controller term is nulled which reduces the above equation to

$$
y=\hat{C x}+E \hat{u}
$$

Using $(2-53)$ in $(2-65)$ gives

$$
\hat{v}_{c a}=D^{\prime} \hat{v}_{p a}
$$

From equation $(2-66)$ it is evident that $\hat{i}_{c}$ has no effect on this output. The other parameters are found in the same manner. It would be rather tedious to go through all of the parameters in detail. Therefore, the solutions have been given below:

$$
\begin{aligned}
& z_{i}=0 \\
& h_{f}=D^{\prime} \\
& y_{o}=0
\end{aligned}
$$

The model can then be reduced to the one shown in Figure $2-9$.

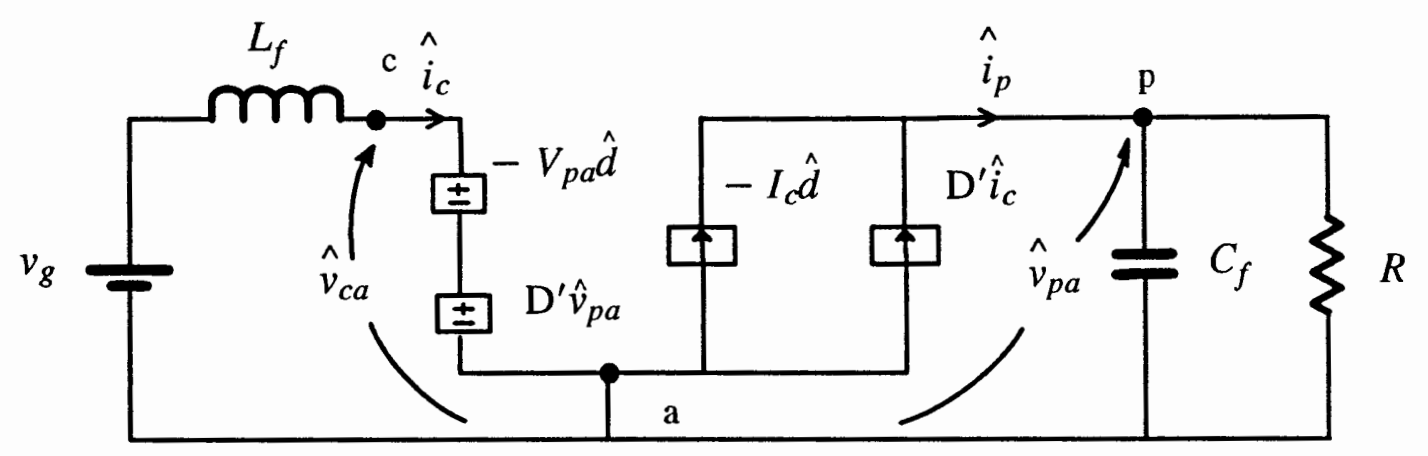

Figure 2 - 9 Hybrid parameter boost model using state space averaging results.

To verify the state space averaging results, the circuit averaging technique will be used to obtain the same model as depicted in Figure 2 - 9. In the circuit averaging method, the current and the voltage waveforms are averaged over one switching period $T_{S}$. The waveforms of the PWM switch of the boost converter of Figure $2-10$ have 
been depicted in Figure $2-11$.

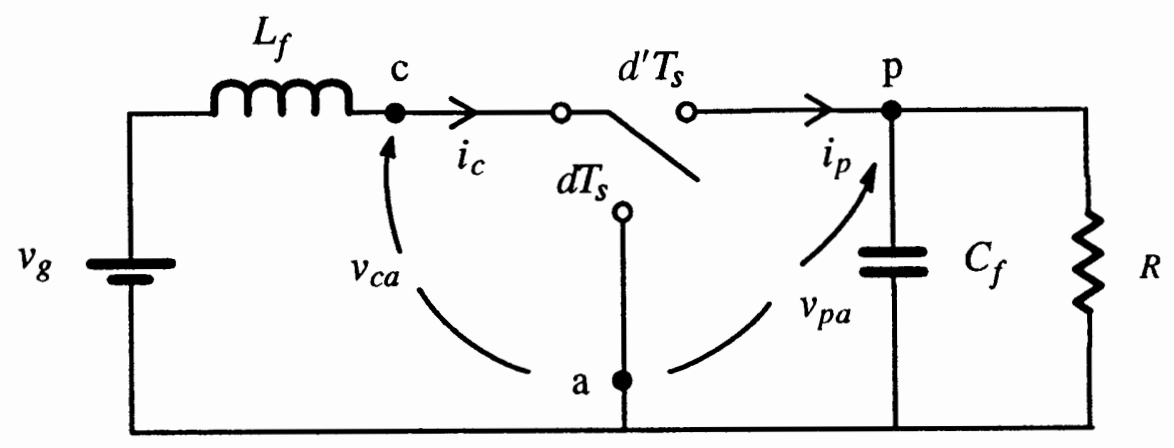

Figure 2 - 10 Typical PWM boost converter.
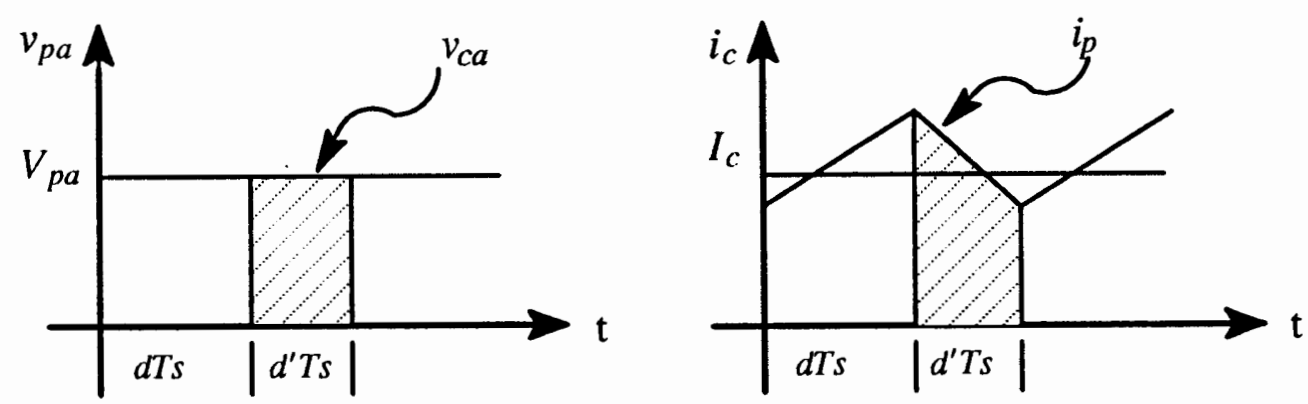

Figure 2-11 The terminal voltages and currents of the switch implementation of Figure $2-10$.

The relationships between the average terminal currents and voltages of the switch are given by

$$
v_{c a}=v_{p a} d^{\prime}
$$

and

$i_{p}=i_{c} d^{\prime}$ 
Figure $2-10$ is thus electrically equivalent to Figure $2-12$.

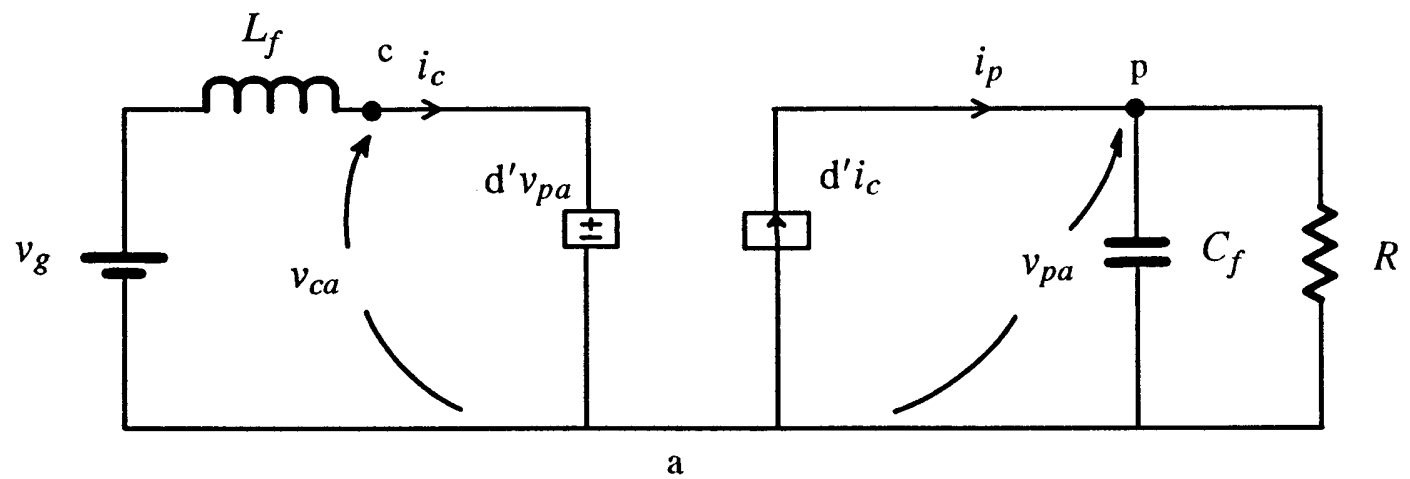

Figure 2-12 Averaged model of Figure 2- 10 .

Perturbing and linearizing equations $(2-70)$ and $(2-71)$ gives

$$
\begin{aligned}
& v_{c a}=\left(V_{p a}+\hat{v}_{p a}\right)\left(D^{\prime}-\hat{d}\right)=D^{\prime} V_{p a}-\hat{d} V_{p a} \\
& i_{p}=\left(I_{c}+\hat{i_{c}}\right)\left(D^{\prime}-\hat{d}\right)=D^{\prime} I_{c}-\hat{d} I_{c}
\end{aligned}
$$

The final circuit averaging model is depicted in Figure $2-13$. It can be observed that this model is identical to the hybrid parameter switch model presented earlier in Figure $2-9$

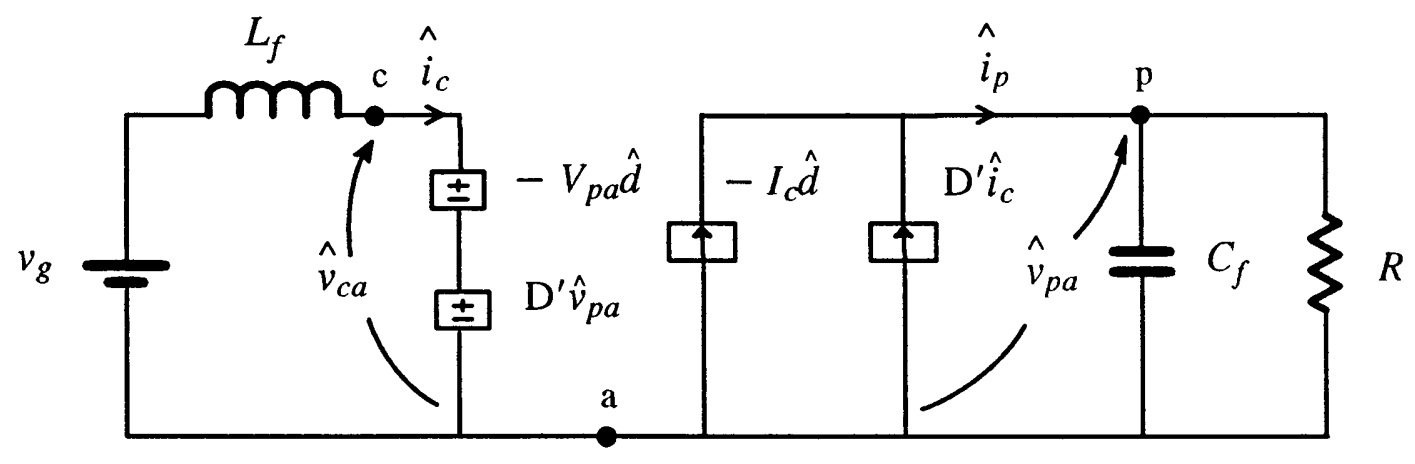

Figure 2-13 Small signal equivalent circuit model for the boost converter of Figure $2-10$.

In the following chapters, different approaches are taken to accurately obtain expressions for the parameters of the hybrid parameter switch model. 
CHAPTER 3

\section{EXACT SMALL SIGNAL ANALYSIS OF PWM CONVERTERS}

It was mentioned earlier that an exact small-signal switch model can be developed using the exact small - signal frequency analysis technique [ 1 ] - [ 3 ]. In section 3.1, the general analysis of switching converters is reviewed. In the next section, a general expression for the exact small-signal response of any piecewise linear network is given. This expression is further simplified for two specific cases:

a. Duty ratio programmed PWM converters operating in the continuous conduction mode (CCM)

b. Current programmed PWM converters operating in CCM.

\subsection{Analysis of Switching Converters in Continuous Conduction Mode}

A dc-to-dc converter operating in Continuous Conduction Mode (CCM) cycles through two switched topologies during each switching period. Since an ideal dc-todc converter is periodic and piecewise linear, it can be described by the following Linear Time Invariant (LTI) state equations:

$\dot{x}(t)=A_{i} x(t)+B_{i} u(t)$

$y(t)=C_{i}^{T} x(t)+E_{i} u(t)$

$t_{i}<t<t_{i+1} \quad i \in(1,2)$ where $t_{i}$ and $t_{i+1}$ are the beginning and the ending times of each subinterval. The switching period is: 


$$
T_{s}=\sum_{i=1}^{2}\left(t_{i+1}-t_{i}\right)
$$

Dc-to-dc converters are controlled by changing the switching times, $t_{i}$ and $t_{i+1}$, such that

$$
t_{i}=T_{i}+\hat{t}_{i}
$$

and

$$
t_{i+1}=T_{i+1}+\hat{t}_{i+1}
$$

The complete solution of the state equation $(3-1)$ at the end of each subinterval is:

$$
x\left(t_{i+1}\right)=e^{A_{i}\left(t_{i+1}-t_{i}\right)} x\left(t_{i}\right)+\int_{t_{i}}^{t_{i+1}} e^{A_{i}\left(t_{i+1}-\tau\right)} B_{i} u(\tau) d \tau
$$

For small signal analysis, the fluctuating line voltage $u(t)$ needs be be separated into the steady state component and the small signal perturbations.

$$
u(t)=U+\hat{u}_{k}(t)=U+\hat{u} e^{j \omega t}
$$

Substituting $(3-7)$ in equation $(3-6)$ gives

$$
x\left(t_{i+1}\right)=e^{A_{i}\left(t_{i+1}-t_{i}\right)} x\left(t_{i}\right)+\int_{t_{i}}^{t_{i+1}} e^{A_{i}\left(t_{i+1}-\tau\right)} B_{i} U d \tau+\int_{t_{i}}^{t_{i+1}} e^{A_{i}\left(t_{i+1}-\tau\right)} B_{i} \hat{u} e^{j \omega \tau} d \tau
$$

Evaluating the integral in equation $(3-8)$ will give

$$
\begin{aligned}
x\left(t_{i+1}\right)= & e^{A_{i}\left(t_{i+1}-t_{i}\right)} x\left(t_{i}\right)+A_{i}^{-1}\left(e^{A_{i}\left(t_{i+1}-t_{i}\right)}-I\right) B_{i} U \\
& +\left(j \omega I-A_{i}\right)^{-1} e^{j \omega t_{i+1}}\left(I-e^{-\left(j \omega I-A_{i}\right)\left(t_{i+1}-t_{i}\right)}\right) B_{i} \hat{u}
\end{aligned}
$$

The state $x(t)$ in each subinterval can be expressed as

$$
x(t)=X(t)+\hat{x}(t)
$$


In the above equation the steady state solution $X(t)$ is periodic within each switching period $\left(T_{s}\right)$. The perturbation term $\hat{x}(t)$ is due to the perturbations in the input, $\hat{u}$ and switching times, $\hat{t}_{i}$ and $\hat{t}_{i+1}$. Figure $3-1$ illustrates one possible way to show states and switching times.

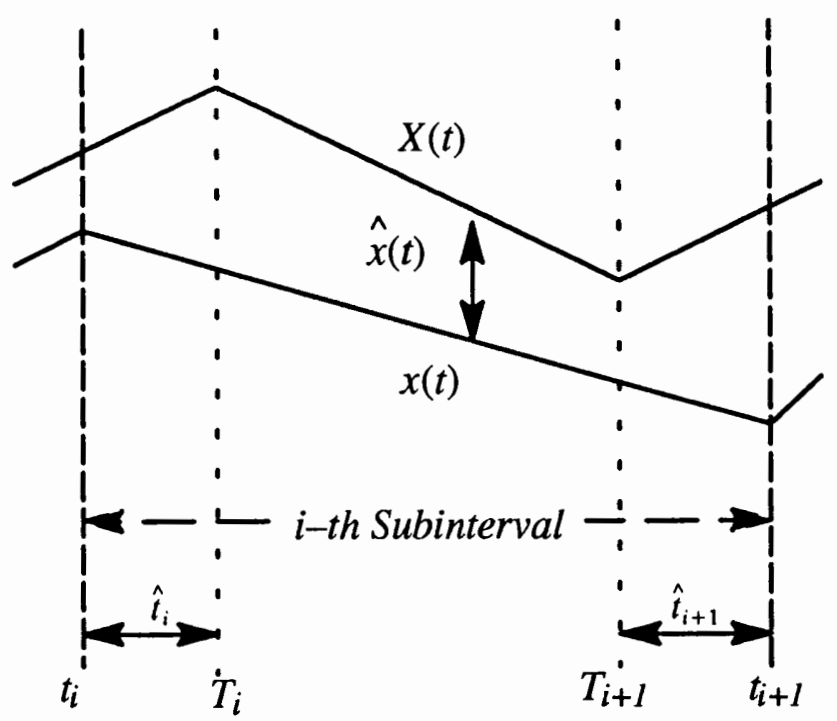

Figure $3-1$ State and Switching times of the $i-t h$ subinterval.

Substituting $(3-4)$ into $(3-10)$ results in:

$x\left(t_{i}\right)=X\left(T_{i}+\hat{t}_{i}\right)+\hat{x}\left(T_{i}+\hat{t}_{i}\right)$

Taylor expansions of $x\left(t_{i}\right)$ at $T_{i}$ is

$x\left(t_{i}\right)=X\left(T_{i}\right)+\dot{X}\left(T_{i}\right) \hat{t}_{i}+\hat{x}\left(T_{i}\right)+\hat{\dot{x}}\left(T_{i}\right) \hat{t}_{i}$

where $X\left(T_{i}\right)$ is the steady state term, $\dot{X}\left(T_{i}\right) \hat{t}_{i}+\hat{x}\left(T_{i}\right)$ are the first order perturbations, and $\hat{\dot{x}}\left(T_{i}\right) \hat{t}_{i}$ is the product of perturbations.

Using $A_{i} X\left(T_{i}\right)+B_{i} U$ in place of $\dot{X}\left(T_{i}\right)$ in equation (3-12) and ignoring the high order perturbation terms, results in:

$x\left(t_{i}\right)=X\left(T_{i}\right)+\left[A_{i} X\left(T_{i}\right)+B_{i} U\right] \hat{t}_{i}+\hat{x}\left(T_{i}\right)$ 
Substituting $(3-13)$ in $(3-9)$ yields

$$
\begin{gathered}
x\left(t_{i+1}\right)=e^{A_{i}\left(t_{i+1}-t_{i}\right)}\left[X\left(T_{i}\right)+\left[A_{i} X\left(T_{i}\right)+B_{i} U\right] \hat{t}_{i}+\hat{x}\left(T_{i}\right)\right]+A_{i}^{-1}\left(e^{A_{i}\left(t_{i+1}-t_{i}\right)}-I\right) B_{i} U \\
+\left(j \omega I-A_{i}\right)^{-1} e^{j \omega t_{i+1}}\left(I-e^{-\left(j \omega I-A_{i}\right)\left(t_{i+1}-t_{i}\right)}\right) B_{i} \hat{u}
\end{gathered}
$$

where $e^{A_{i}\left(t_{i+1}-t_{i}\right)}$ can be expanded into the steady state and perturbation terms:

$$
e^{A_{i}\left(t_{i+1}-t_{i}\right)}=e^{A_{i}\left(T_{i+1}-T_{i}\right)} e^{A_{i}\left(\hat{t}_{i+1}-\hat{t}_{i}\right)}
$$

Linearizing the exponential perturbation component in expression (3 - 15), using a first order taylor series expansion, gives:

$e^{A_{i}\left(t_{i+1}-t_{i}\right)}=e^{A_{i}\left(T_{i+1}-T_{i}\right)}\left[I+A_{i}\left(\hat{t}_{i+1}-\hat{t}_{i}\right)\right]$

Using the result of $(3-16)$ in equation $(3-14)$ and rearranging it further gives

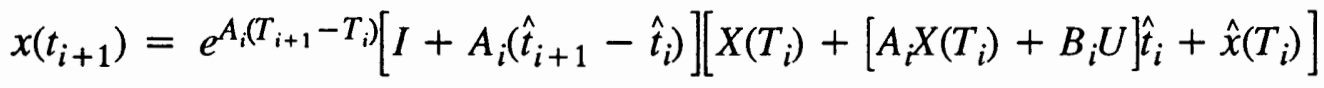

$$
\begin{aligned}
& +A_{i}^{-1}\left(e^{A_{i}\left(T_{i+1}-T_{i}\right)}\left[I+A_{i}\left(\hat{t}_{i+1}-\hat{t}_{i}\right)\right]-I\right) B_{i} U \\
& +\left(j \omega I-A_{i}\right)^{-1} e^{j \omega T_{i+1}\left(1+j \omega \hat{t}_{i+1}\right)} . \\
& \left(I-e^{-\left(j \omega I-A_{i}\right)\left(T_{i+1}-T_{i}\right)}\left[I-\left(j \omega I-A_{i}\right)\left(\hat{t}_{i+1}-\hat{t}_{i}\right)\right]\right) B_{i} \hat{u}
\end{aligned}
$$

Changing the subscripts in equation $(3-13)$ to $i+1$ gives

$$
x\left(t_{i+1}\right)=X\left(T_{i+1}\right)+\left[A_{i+1} X\left(T_{i+1}\right)+B_{i+1} U\right] \hat{t}_{i+1}+\hat{x}\left(T_{i+1}\right)
$$

and substituting equation $(3-18)$ in the left hand side of equation $(3-17)$ will give:

$$
\begin{aligned}
& X\left(T_{i+1}\right)+\left[A_{i+1} X\left(T_{i+1}\right)+B_{i+1} U\right] \hat{t}_{i+1}+\hat{x}\left(T_{i+1}\right)=
\end{aligned}
$$

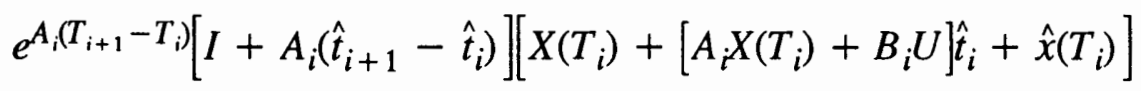

$$
\begin{aligned}
& +A_{i}^{-1}\left(e^{A_{i}\left(T_{i+1}-T_{i}\right)}\left[I+A_{i}\left(\hat{t}_{i+1}-\hat{t}_{i}\right)\right]-I\right) B_{i} U \\
& +\left(j \omega I-A_{i}\right)^{-1} e^{j \omega T_{i+1}\left(1+j \omega \hat{t}_{i+1}\right)} . \\
& \left(I-e^{-\left(j \omega I-A_{i}\right)\left(T_{i+1}-T_{i}\right)}\left[I-\left(j \omega I-A_{i}\right)\left(\hat{t}_{i+1}-\hat{t}_{i}\right)\right]\right) B_{i} \hat{u}
\end{aligned}
$$


Only the zeroth and first order terms of this equation are of interest. Therefore, the steady state and the first order term of the state at the end of the $i-t h$ subinterval can be obtained by seperating the approprite terms and simplifying the results. From equation $(3-19)$ the zeroth order term is:

$$
\begin{array}{r}
x\left(T_{i+1}\right)=e^{A_{i}\left(T_{i+1}-T_{i}\right)} X\left(T_{i}\right)+A_{i}^{-1}\left(e^{A_{i}\left(T_{i+1}-T_{i}\right)}-I\right) B_{i} U \\
\text { provided det } A_{i} \neq 0
\end{array}
$$

and first order term is:

$$
\begin{aligned}
& \hat{x}\left(T_{i+1}\right)=e^{A_{i}\left(T_{i+1}-T_{i}\right)} \hat{x}\left(T_{i}\right)+e^{A_{i}\left(T_{i+1}-T_{i}\right)}\left[A_{i} X\left(T_{i}\right)+B_{i} U\right] \hat{t}_{i+1} \\
& -\left[A_{i+1} X\left(T_{i+1}\right)+B_{i+1} U\right] \hat{t}_{i+1} \\
& +\left(j \omega I-A_{i}\right)^{-1} e^{j \omega T_{i+1}}\left(I-e^{-\left(j \omega I-A_{i}\right)\left(T_{i+1}-T_{i}\right)}\right) B_{i} \hat{u}
\end{aligned}
$$

The second term on the right hand side of equation $(3-21)$ can be written in terms of $X\left(T_{i+1}\right)$. Premultiplying equation $(3-20)$ by $e^{-A_{i}\left(T_{i+1}-T_{i}\right)}$ gives:

$$
x\left(T_{i}\right)=e^{-A_{i}\left(T_{i+1}-T_{i}\right)} X\left(T_{i+1}\right)+e^{-A_{i}\left(T_{i+1}-T_{i}\right)} A_{i}{ }^{-1}\left(e^{A_{i}\left(T_{i+1}-T_{i}\right)}-I\right) B_{i} U
$$

where the comutitativity property of a a matrix and its exponential has been used.

Substituting equation $(3-22)$ in $(3-21)$ will give:

$$
\begin{gathered}
\hat{x}\left(T_{i+1}\right)=e^{A_{i}\left(T_{i+1}-T_{i}\right)} \hat{x}\left(T_{i}\right)+\left[\left(A_{i}-A_{i+1}\right) X\left(T_{i+1}\right)+\left(B_{i}-B_{i+1}\right) U\right] \hat{t}_{i+1} \\
+\left(j \omega I-A_{i}\right)^{-1} e^{j \omega T_{i+1}}\left(I-e^{-\left(j \omega I-A_{i}\right)\left(T_{i+1}-T_{i}\right)}\right) B_{i} \hat{u}
\end{gathered}
$$

Equation $(3-23)$ can be written as:

$$
\hat{x}\left(T_{i+1}\right)=\Phi_{i} \hat{x}\left(T_{i}\right)+\xi_{i} \hat{t}_{i+1}+\eta_{i} \hat{u}_{k}\left(T_{i+1}\right)
$$

where

$$
\begin{aligned}
& \Phi_{i}=e^{A_{i}\left(T_{i+1}-T_{i}\right)} \\
& \xi_{i}=\left[\left(A_{i}-A_{i+1}\right) X\left(T_{i+1}\right)+\left(B_{i}-B_{i+1}\right) U\right]
\end{aligned}
$$




$$
\begin{aligned}
& \eta_{i}=\left(j \omega I-A_{i}\right)^{-1}\left(I-e^{-\left(j \omega I-A_{i}\right)\left(T_{i+1}-T_{i}\right)}\right) B_{i} \\
& \hat{u}_{k}\left(T_{i+1}\right)=\hat{u} e^{j \omega T_{i+1}}
\end{aligned}
$$

For small signal analysis, the controller signal is expressed as:

$r\left(t_{i}\right)=R+\hat{r}_{k}\left(t_{i}\right)=R+\hat{r}_{p} e^{j \omega t}$

The difference equation $(3-24)$ has a time perturbation term $\xi_{i} \hat{t}_{i+1}$ which makes the system time varying. This term must be evaluated and replaced by appropriate linear combination of states and inputs. For example, for a current mode programming PWM converter operaing in CCM, the control input equals a linear combination of states, $h_{i}^{T} x\left(\hat{t}_{i+1}\right)$, plus a ramp with slope $m_{i}$.

$$
\hat{r}\left(t_{i+1}\right)=h_{i}^{T} \hat{x}\left(t_{i+1}\right)+m_{i} \hat{t}_{i+1}
$$

Next, equation $(3-24)$ is written in terms of $x\left(\hat{t}_{i+1}\right)$. Using only the perturbation terms of equation $(3-18)$ gives

$$
\hat{x}\left(t_{i+1}\right)=\Phi_{i} \hat{x}\left(T_{i}\right)+\gamma_{i} \hat{t}_{i+1}+\eta_{i} \hat{u}_{k}\left(T_{i+1}\right)
$$

where $\gamma_{i}=A_{i} X\left(T_{i+1}\right)+B_{i} U$.

Equations $(3-30)$ and $(3-31)$ are then used together to give the desired state difference equations. The derivations for these equations are given in [3].

\subsection{Application of Time Varying Transfer Function}

Using the above analysis, it can be shown [ 3 ] that the exact small signal frequency response of any piecewise linear network can be obtained in the general form given as:

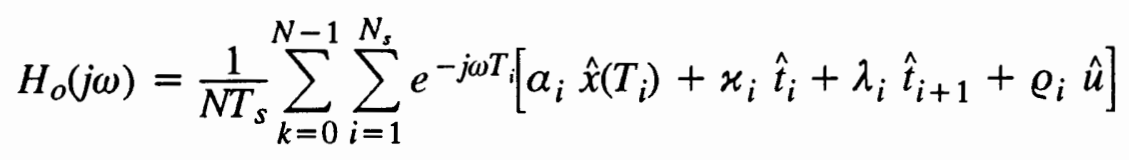

where

$$
\alpha_{i}=C_{i}^{T}\left(j \omega I-A_{i}\right)^{-1}\left[I-e^{-\left(j \omega I-A_{i}\right)\left(T_{i+1}-T_{i}\right)}\right]
$$




$$
\begin{aligned}
& \varkappa_{i}=-\left[C_{i}^{T} X\left(T_{i}\right)+E_{i} U\right] \\
& \lambda_{i}=\left[C_{i}^{T} X\left(T_{i}\right)+E_{i} U\right] e^{-j \omega\left(T_{i+1}-T_{i}\right)} \\
& \varrho_{i}=e^{j \omega T_{i}}\left\{C_{i}^{T}\left(j \omega I-A_{i}\right)^{-1}\left[\left(T_{i+1}-T_{i}\right) B_{i}-\eta_{i}\right]+E_{i}\left(T_{i+1}-T_{i}\right)\right\}
\end{aligned}
$$

where $N$ is the number of cycles of the switching waveform and $N_{s}$ is the number of topologies per switching period.

Expression $(3-32)$ can be used to analyze any piecewise linear network. In the following, the exact small-signal transfer functions for PWM converters in CCM under duty ratio, and current mode programming are shown.

\subsubsection{Duty Ratio Programmed PWM Converter in CCM}

For duty ratio programming, the control voltage $v_{c}$ is compared with a positive sawtooth ramp $v_{r}$. The duty ratio of the $k$ th cycle is defined as:

$$
d_{k}=D+\hat{d}_{k}
$$

The control signal is given in the following form

$$
\begin{aligned}
& v_{c}=V_{c}+\hat{v}_{c} \\
& \hat{v}_{c}=v_{c p} \cos \left[(k+\delta) \omega T_{s}\right]
\end{aligned}
$$

where

$$
\begin{array}{ll}
\delta=d_{k} ; & \text { for natural sampling } \\
\delta=0 ; & \text { for uniform sampling }
\end{array}
$$

The sawtooth ramp has a peak - to-peak value $V_{r}$ and minimum value $V_{o}$. The intersection of the ramp with the control signal determines the width of the duty ratio. Duty ratio directly turns the active switch ON. The duty ratio of the $k$ th cycle is determined as:

$$
\frac{V_{r}}{T_{s}}\left(d_{k} T_{s}\right)+V_{o}=v_{c}
$$


This arrangement is shown in Figure $3-2$.

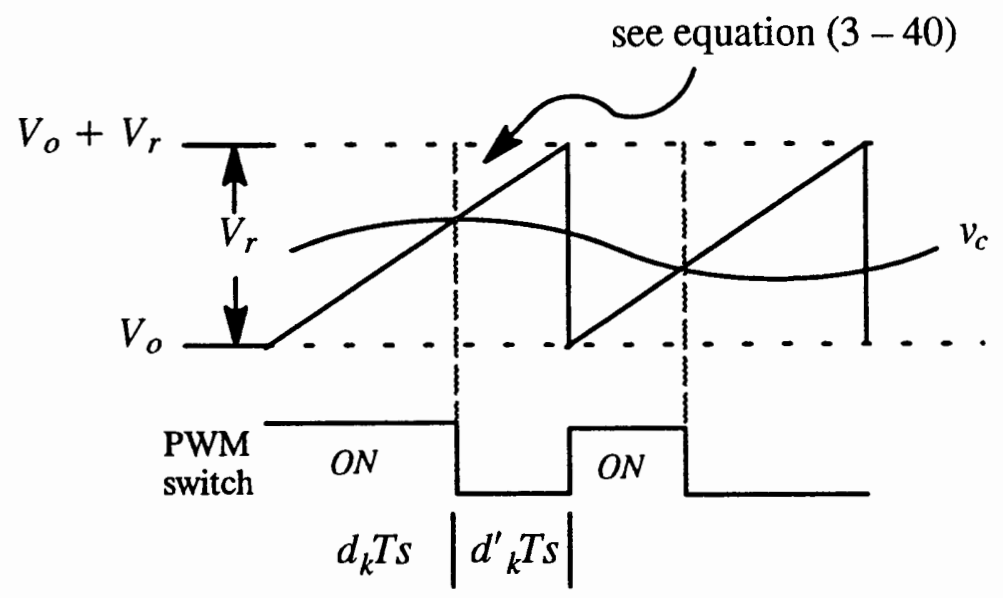

Figure $3-2$ Waveforms showing typical signals associated with duty ratio determination.

Equation ( $3-40)$ can be divided into dc and first order perturbation. The dc term of the above relationship is found as:

$$
D=\frac{V_{c}-V_{o}}{V_{r}}
$$

The the small signal duty ratio is:

$$
\hat{d}_{k}=\frac{\hat{v}_{c}}{V_{r}}
$$

Next, the exact small signal frequency response transfer functions are given. In the following chapter, these equations are used to obtain exact expressions for the PWM duty ratio programmed hybrid parameter switch model.

$$
\frac{\hat{y}}{\hat{d}}(j \omega)=\left[\alpha_{1} \beta_{2}+\alpha_{2}\right]\left(I-\beta_{1} \beta_{2}\right)^{-1} \xi+\zeta
$$




$$
\begin{aligned}
\frac{\hat{y}}{\hat{u}}(j \omega)=\frac{1}{T_{s}}\left\{\left[\alpha_{1} \beta_{2}\right.\right. & \left.+\alpha_{2}\right]\left(I-\beta_{1} \beta_{2}\right)^{-1}\left(\beta_{2} \eta_{1}+\eta_{2}\right) \\
+ & \alpha_{1} \eta_{2}+E_{1}\left(T_{2}-T_{1}\right)+E_{2}\left(T_{3}-T_{2}\right) \\
& +C_{1}\left(j \omega I-A_{1}\right)^{-1}\left[\left(T_{2}-T_{1}\right) B_{1}-\eta_{1}\right] \\
& \left.+C_{2}\left(j \omega I-A_{2}\right)^{-1}\left[\left(T_{3}-T_{2}\right) B_{2}-\eta_{2}\right]\right\}
\end{aligned}
$$

where

$$
\begin{aligned}
a_{i}= & C_{i}\left(j \omega I-A_{i}\right)^{-1}\left[I-e^{\left.-\left(j \omega I-A_{i}\right)\left(T_{i+1}-T_{i}\right)\right]}, \quad i=1,2\right. \\
\beta_{i}= & e^{-\left(j \omega I-A_{i}\right)\left(T_{i+1}-T_{i}\right)}, \quad i=1,2 \\
\eta_{i}= & \left(j \omega I-A_{i}\right)^{-1}\left[I-e^{-\left(j \omega I-A_{i}\right)\left(T_{i+1}-T_{i}\right)}\right] B_{i}, \quad i=1,2 \\
\xi= & \left(A_{1}-A_{2}\right) X+\left(B_{1}-B_{2}\right) U \\
\zeta= & \left(C_{1}-C_{2}\right) X+\left(E_{1}-E_{2}\right) U \\
X= & {\left[I-e^{A_{1} D T_{s}} e^{A_{2} D^{\prime} T_{s}}\right]^{-1}\left\{e^{A_{1} D T_{s}}\left[e^{A_{2} D^{\prime} T_{s}}-I\right]\right.} \\
& \left.. A_{2}^{-1} B_{2} U+\left[e^{A_{1} D T_{s}}-I\right] A_{1}^{-1} B_{1} U\right\}
\end{aligned}
$$

\subsubsection{Current Programmed Converter in CCM}

In current programming, the switch is turned $\mathrm{ON}$ when the voltage ramp $R_{i} i_{\zeta}$ reaches the control signal $v_{c}$. The above voltage ramp is proportional to the instantaneous sensed inductor current ramp. The current to voltage transformation gain $R_{i}$ is used to show this proportionality. Therefore, switch turn $\mathrm{ON}$ is dependent on the control signal and the sensed current. This is shown in Figure $3-3$ : 


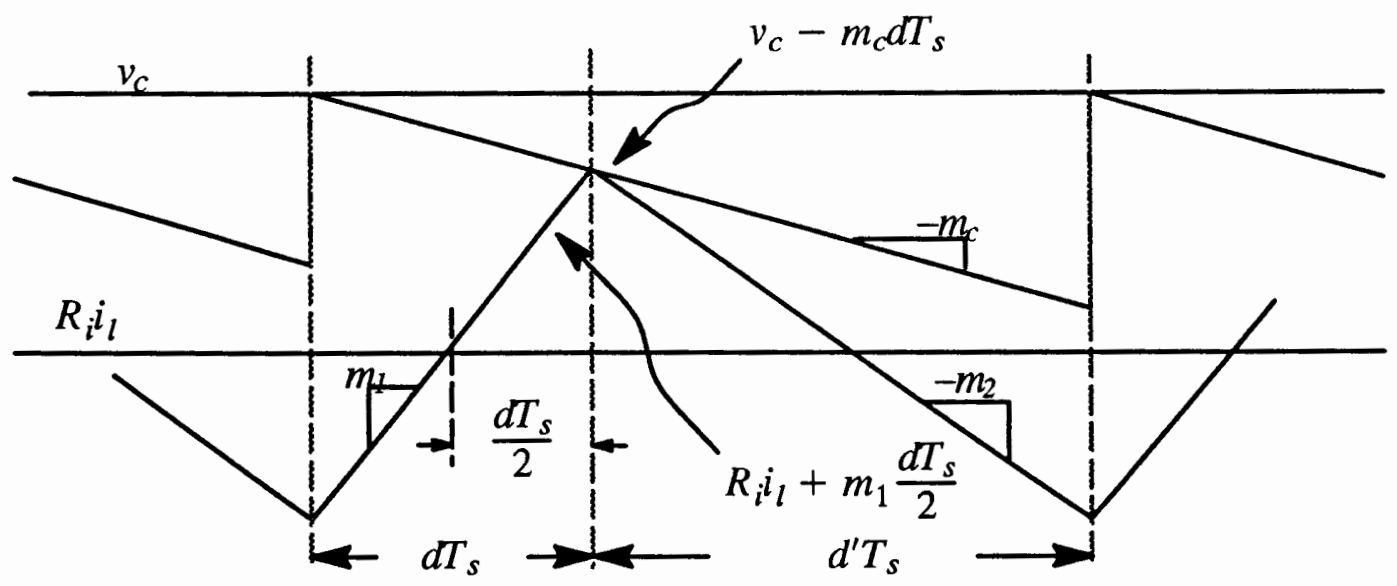

Figure 3-3 Waveforms showing the intersection of voltage ramps that cause switching to take place.

The exact small-signal describing functions given in [ 3 ] can be used to obtain the control-to-output and the input-to-output transfer functions for converters operating in continuous conduction mode under current mode programming.

The control-to-output transfer function given in terms of control voltage is:

$\frac{\hat{y}}{\hat{v_{c}}}(j \omega)=\frac{\mu}{T_{s}}\left\{\left[\alpha_{1} \beta_{2}+\alpha_{2}-\xi h^{T} \mu \beta_{1} \beta_{2}\right]\left(I-\Gamma \beta_{1} \beta_{2}\right)^{-1} \xi+\zeta\right\}$

where $\frac{\mu}{T_{s}}$ is the modulator gain.

The input-to-output transfer function is given in the following form:

$$
\begin{aligned}
\frac{\hat{y}}{\hat{u}}(j \omega)= & \frac{1}{T_{s}}\left\{\left(\left[\alpha_{1} \beta_{2}+\alpha_{2}-\zeta h^{T} \mu \beta_{1} \beta_{2}\right]\left(I-\Gamma \beta_{1} \beta_{2}\right)^{-1} \Gamma-\zeta h^{T} \mu\right)\right. \\
& .\left(\beta_{2} \eta_{1}+\eta_{2}\right)+\alpha_{1} \eta_{2}+E_{1}\left(T_{2}-T_{1}\right)+E_{2}\left(T_{3}-T_{2}\right) \\
& +C_{1}\left(j \omega I-A_{1}\right)^{-1}\left[\left(T_{2}-T_{1}\right) B_{1}-\eta_{1}\right] \\
& \left.+C_{2}\left(j \omega I-A_{2}\right)^{-1}\left[\left(T_{3}-T_{2}\right) B_{2}-\eta_{2}\right]\right\}
\end{aligned}
$$


where

$$
\begin{aligned}
\mu & =\frac{1}{h^{T}\left[A_{1} X+B_{1} U\right]+m_{c}} \\
\Gamma & =I-\xi h^{T} \mu
\end{aligned}
$$

and $\left\{\alpha_{i}, \beta_{i}, \eta_{i}, \xi, \zeta, X\right\}$ are the same as those given for PWM CCM analysis. 


\section{CHAPTER 4}

\section{FIRST MODEL}

\subsection{PWM Duty Ratio Programmed Model}

In chapter 3, the general form of the hybrid parameter switch model was introduced. Next, the state-space averaged control-to-output and the input-tooutput transfer functions were used to obtain expressions for the parameters of the model. The subsequent model was found to agree with the circuit averaging model. In the sequel, the exact expressions for the parameters will be sought for using the exact small signal transfer functions given in equations $(3-43)$ and $(3-44)$.

In the first step, the small - signal state equation of the boost model of Figure $2-7$ will be obtained. The two independent sources of this model are actually the states of the system. Therefore,

$$
\hat{i}_{c}=\hat{i}
$$

and

$$
\hat{v}_{p a}=\hat{v}
$$

Using Kirchoff's voltage law (KVL) one gets:

$$
-\hat{v}_{g}+\frac{\hat{d i}}{d t} L+v_{i d} \hat{d}+h_{r} \hat{v}_{p a}+z_{i} \hat{i}_{c}=0
$$

Substituting equations $(4-1)$ and $(4-2)$ in $(4-3)$ and rearranging gives:

$$
\frac{\hat{d i}}{d t}=-\frac{v_{i d}}{L_{f}} \hat{d}-\frac{z_{i}}{L_{f}} \hat{i}+\frac{1-h_{r}}{L_{f}} \hat{v}_{g}
$$


Applying Kirchoff's current law (KCL) to the $p$ terminal of Figure $2-7$ will give:

$\frac{\hat{d v}}{d t} C_{f}+\frac{\hat{v}_{p a}}{R}-\hat{i}_{p}=0$

Using KCL again gives:

$$
\hat{i}_{p}=i_{o d} \hat{d}+h_{f} \hat{i}_{c}+y_{o} \hat{v}_{p a}
$$

Substituting equations $(4-1),(4-2)$ in equation $(4-6)$ and substituting the results in equation $(4-5)$ will give:

$$
\frac{d \hat{v}}{d t}=\frac{i_{o d}}{C_{f}} \hat{d}+\frac{h_{f}}{C_{f}} \hat{i}+\left(\frac{y_{o}-\frac{1}{R}}{C_{f}}\right) \hat{v}
$$

Equations $(4-4)$ and $(4-7)$ together form the small-signal state equation of the system:

$$
\frac{d}{d t}\left[\begin{array}{l}
\hat{i} \\
\hat{v}
\end{array}\right]=\left[\begin{array}{cc}
-\frac{z_{i}}{L_{f}} & -\frac{h_{r}}{L_{f}} \\
\frac{h_{f}}{C_{f}} & \frac{y_{o}}{C_{f}}-\frac{1}{R C_{f}}
\end{array}\right]\left[\begin{array}{l}
\hat{i} \\
\hat{v}
\end{array}\right]+\left[\begin{array}{r}
-\frac{v_{i d}}{L_{f}} \\
\frac{i_{o d}}{C_{f}}
\end{array}\right] \hat{d}+\left[\begin{array}{c}
\frac{1}{L_{f}} \\
0
\end{array}\right] \hat{v}_{g}
$$

In order to obtain the frequency response of equation $(4-8)$, one may simply use state space averaging transfer functions given in equations $(2-49)$ and $(2-50)$.

To verify this modeling approach, the parameters are numerically evaluated at each discrete frequency point using equations $(3-43)$ and $(3-44)$. Then the overall response is formulated using equations $(2-49)$ and $(2-50)$. The plots obtained from the switch model are then compared against the exact solutions of equations $(3-43)$ and $(3-44)$. These plotts are shown in Figure $4-1$ and Figure $4-2$. For the given specifications the ripple percentages are calculated to be:

$$
\begin{aligned}
& \frac{\Delta v / 2}{V} \times 100 \approx 4.85 \% \\
& \frac{\Delta i / 2}{I} \times 100 \approx 37.2 \%
\end{aligned}
$$


where $\Delta v$ and $\Delta i$ are the peak-to-peak capacitor voltage ripple the inductor current ripple, respectively. The quantities $V$ and $I$ are the steady state operating values for the capacitor voltage and inductor current. As it is apparent from Figure 4-1 this model does not reproduce the exact plot for the control-to-output frequency response.

From equations $(2-41)$ and $(2-42)$ it is evident that system states, $\hat{i}_{c}$ and $\hat{v}_{p a}$ are also the independent sources of the system. Therefore, nulling them would eliminate the dynamics of the system and reduces the exact controller parameters $v_{i d}$ and $i_{o d}$ to the state space averaging expressions. To clarify this point, the $v_{i d}$ parameter will be found next.

Using control-to-output transfer function given in equation (3 - 43) and also the constraints given in equation $(2-43) v_{i d}$ is found as follows:

$v_{i d}=\frac{\hat{v}_{c a}}{\hat{d}}=\left[\alpha_{1} \beta_{2}+\alpha_{2}\right]\left(I-\beta_{1} \beta_{2}\right)^{-1} \xi+\zeta \mid \begin{aligned} & \hat{v}_{p a}=0 \\ & \hat{i}_{c}=0\end{aligned}$

where

$$
\begin{aligned}
& \alpha_{1}=C_{1}\left(j \omega I-A_{1}\right)^{-1}\left[I-e^{-\left(j \omega I-A_{1}\right)\left(T_{2}-T_{1}\right)}\right] \\
& \alpha_{2}=C_{2}\left(j \omega I-A_{2}\right)^{-1}\left[I-e^{-\left(j \omega I-A_{2}\right)\left(T_{3}-T_{2}\right)}\right] \\
& \beta_{1}=e^{-\left(j \omega I-A_{1}\right)\left(T_{2}-T_{1}\right)} \\
& \beta_{2}=e^{-\left(j \omega I-A_{2}\right)\left(T_{3}-T_{2}\right)}
\end{aligned}
$$

Using ( $2-53), \alpha_{1}$ and $\alpha_{2}$ for $v_{i d}$ parameter will be:

$$
\begin{aligned}
& \alpha_{1}=\left[\begin{array}{ll}
0 & 0
\end{array}\right]\left(j \omega I-A_{1}\right)^{-1}\left[I-e^{-\left(j \omega I-A_{1}\right)\left(T_{2}-T_{1}\right)}\right] \\
& \alpha_{2}=\left[\begin{array}{ll}
0 & 1
\end{array}\right]\left(j \omega I-A_{2}\right)^{-1}\left[I-e^{-\left(j \omega I-A_{2}\right)\left(T_{3}-T_{2}\right)}\right]
\end{aligned}
$$


Nulling both states will force $C_{1}$ and $C_{2}$ vectors to zero. This is shown below:

$$
\begin{aligned}
& C_{1}=\left[\begin{array}{ll}
0 & 0
\end{array}\right] \\
& C_{2}=\left[\begin{array}{ll}
0 & 0
\end{array}\right]
\end{aligned}
$$

Using $(4-14)$ in $(4-13)$ results in:

$$
\begin{aligned}
& \alpha_{1}=\left[\begin{array}{ll}
0 & 0
\end{array}\right]\left(j \omega I-A_{1}\right)^{-1}\left[I-e^{-\left(j \omega I-A_{1}\right)\left(T_{2}-T_{1}\right)}\right]=0 \\
& \alpha_{2}=\left[\begin{array}{ll}
0 & 0
\end{array}\right]\left(j \omega I-A_{2}\right)^{-1}\left[I-e^{-\left(j \omega I-A_{2}\right)\left(T_{3}-T_{2}\right)}\right]=0
\end{aligned}
$$

Therefore, when both states are nulled, equation $(3-43)$ reduces to:

$$
\frac{\hat{y}}{\hat{d}}(j \omega)=\zeta
$$

Since $\zeta$ is equivalent to $E_{d}$ of the state space averaging method, it can be seen that equation $(4-16)$ is identical to equation $(2-59)$. As a result of this reduction, all of the components contributing to the exactness of the solution will be lost. Therefore, this technique fails to produce exact expressions for the control-to-output parameters of the PWM duty ratio controlled boost converter operating in CCM. As shown in Figure $4-2$, the input-to-output response of the model matches that of the exact results. 

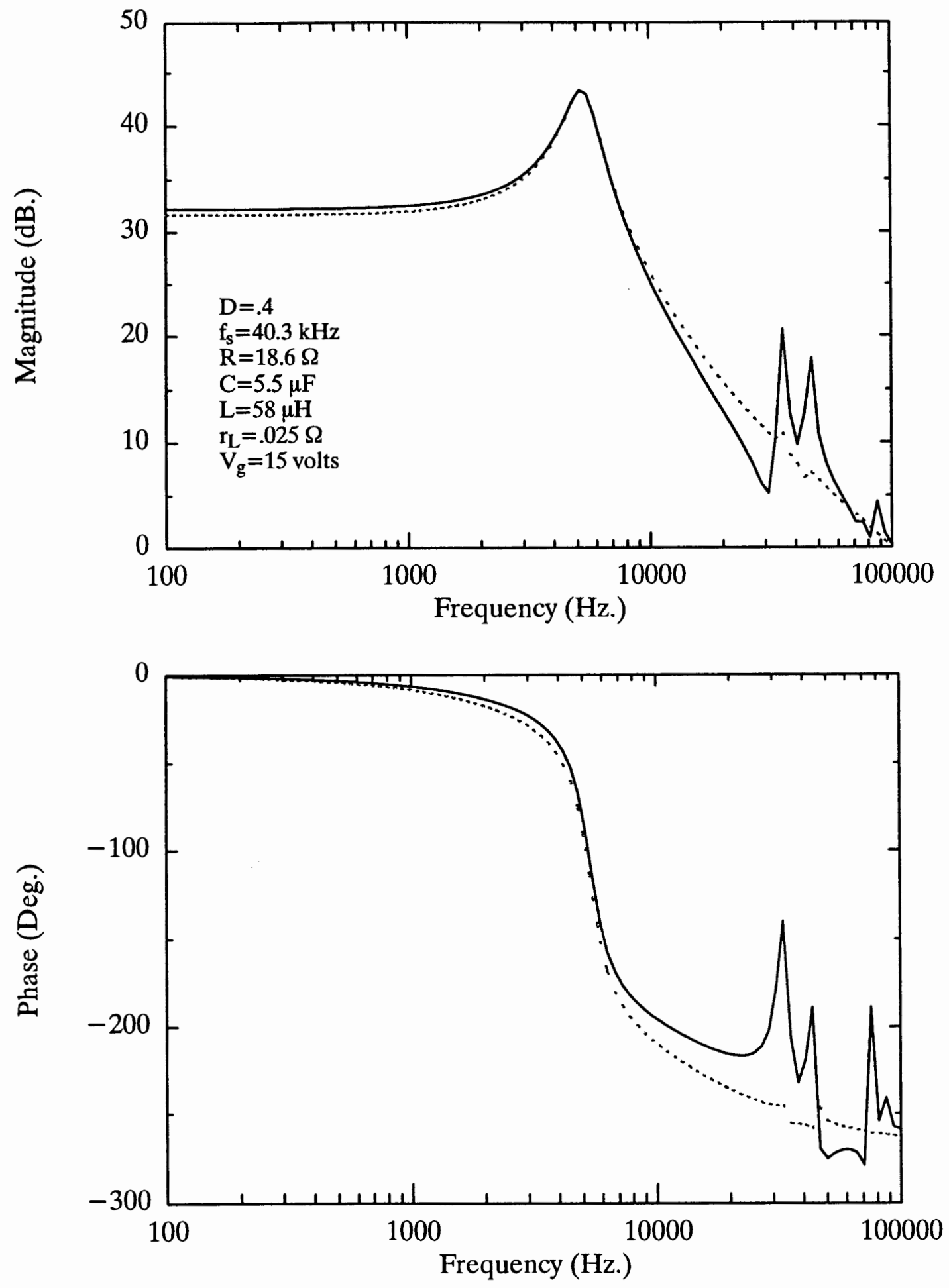

Figure 4-1 The control-to-output response of the PWM boost converter operating in CCM; exact (solid line) versus the first model (dashed line). 

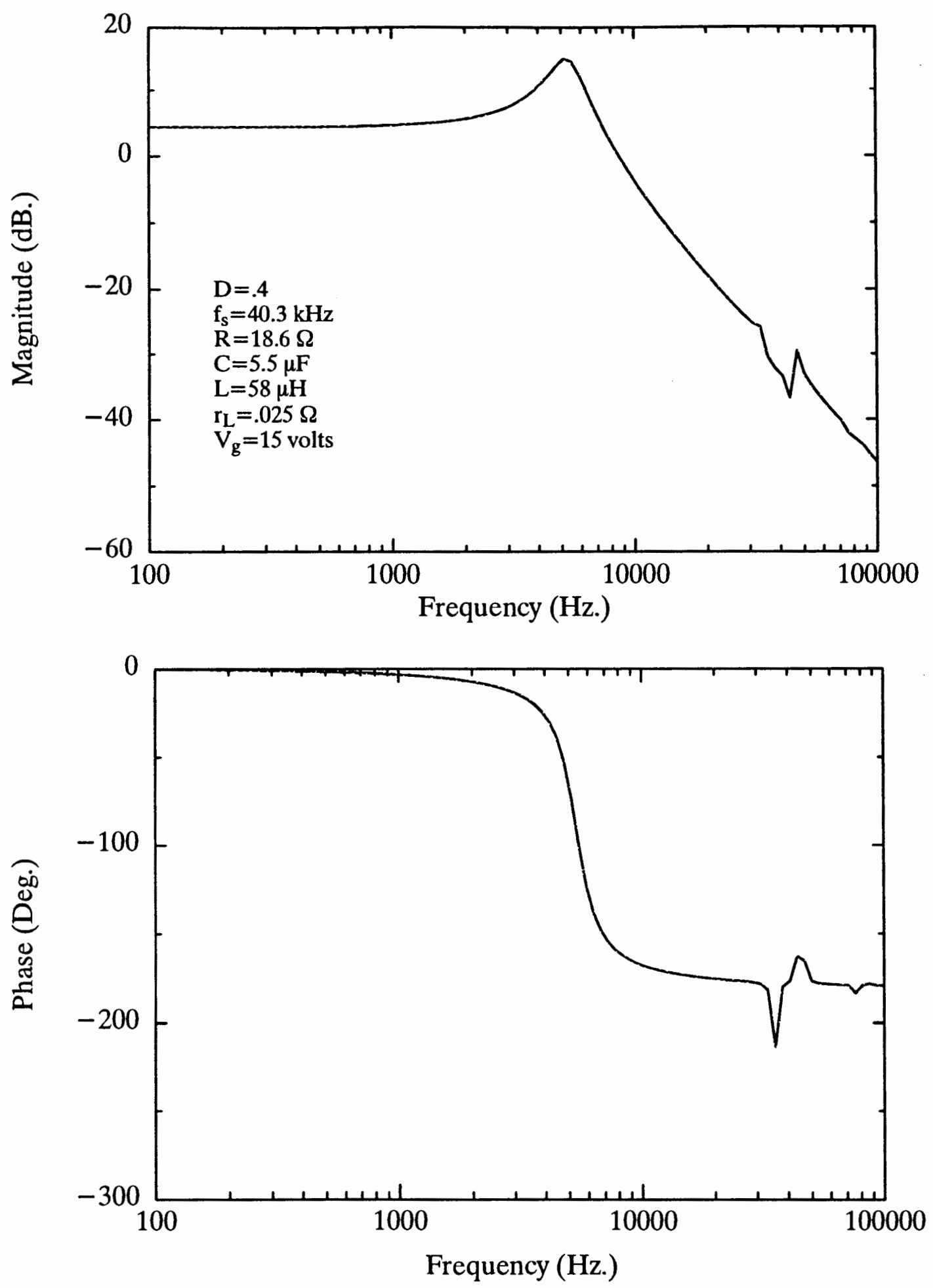

Figure 4-2 The input-to-output response of the PWM boost converter operating in CCM; exact (solid line) versus the first model (dashed line). 


\subsection{PWM Current Programmed Model}

It is apparent from the control-to-output transfer function of the current-programmed converters in equation $(3-51)$ that this method does not work for the current mode programming either. Nulling both states would reduce equation $(3-52)$ to:

$$
\frac{\hat{y}}{\hat{v}_{c}}(j \omega)=\frac{\mu}{T_{s}} \zeta
$$

where $\mu$ is given by equation ( $3-53)$. In terms of duty ratio- to-output transfer function, equation $(4-17)$ reduces to:

$$
\frac{\hat{y}}{\hat{d}}(j \omega)=\zeta
$$

which is equivalent to the state space averaging solution given by equation $(2-59)$.

Generally, the current mode transfer functions given in equations $(3-51)$ and $(3-52)$ are more complex and involve more terms due to the current sensing phenomena. The state space averaging results fail to predict the behavior of the converters under current mode control. The easiest way to verify the results would be through comparing Matlab frequency response plots of the exact small-signal results versus those of the proposed model. These plots are shown in Figure $4-3$ and Figure $4-4$. The following ripple percentages are used in the above simulation:

$\frac{\Delta v / 2}{V} \times 100 \approx 3 \%$

$\frac{\Delta i / 2}{I} \times 100 \approx 35 \%$

In Figure 4-3, the control-to-output response of the model is plotted against the exact results. As shown in the above figure, the magnitude response of the proposed model has an overall level shift of around $+20 \mathrm{~dB}$ up to half the switching frequency. The phase plot of the model shows around $180^{\circ} \mathrm{deg}$. phase lag at low frequencies. 
Obviously, this modeling technique does not predict the exact results. In the next chapter, a new method is proposed which is believed to overcome the inaccuracies associated with this model. 

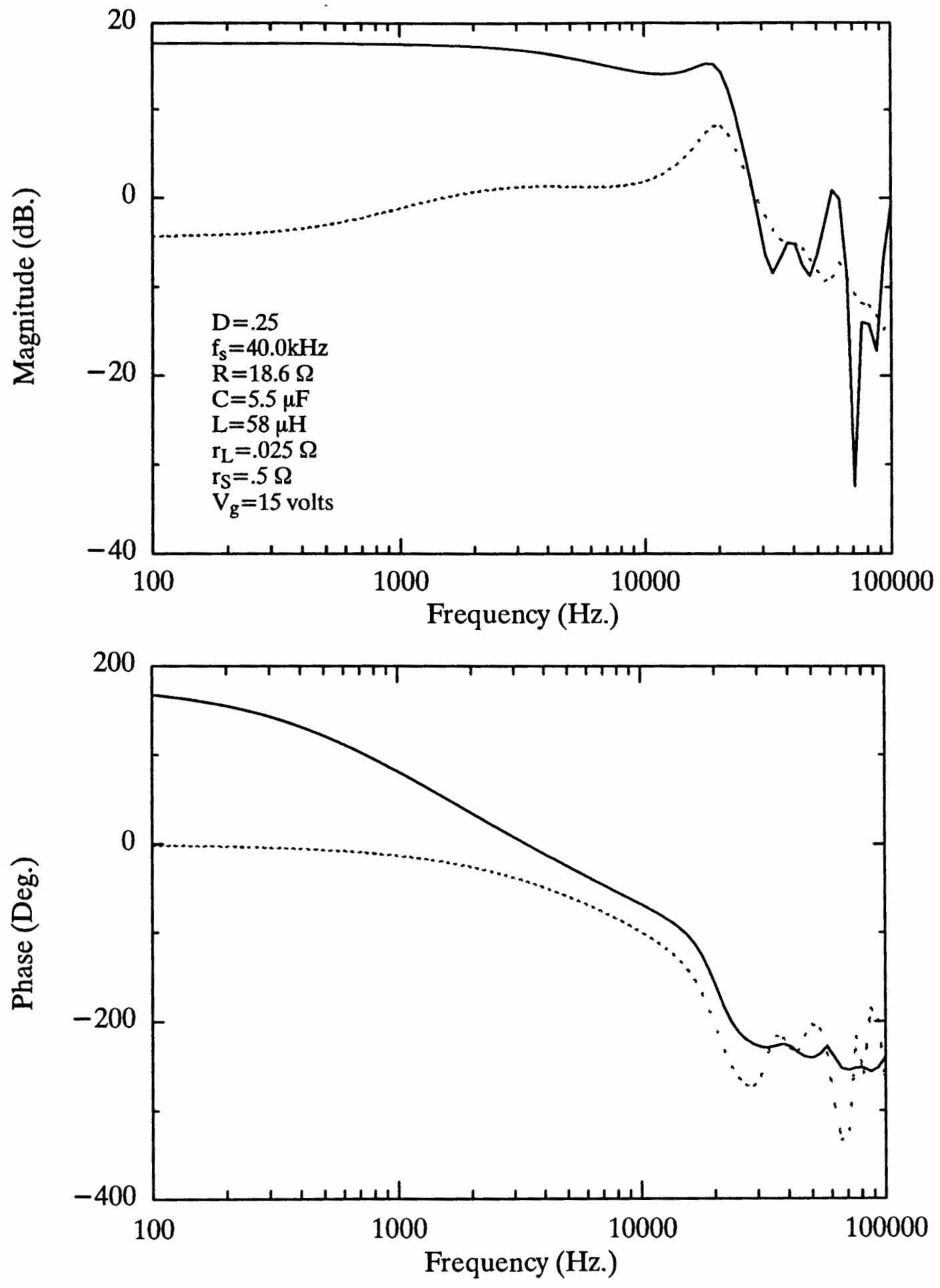

Figure 4-3 The control-to-output response of the PWM boost converter operating in $\mathrm{CCM}$ under current mode programming; exact (solid line) versus the first model (dashed line). 

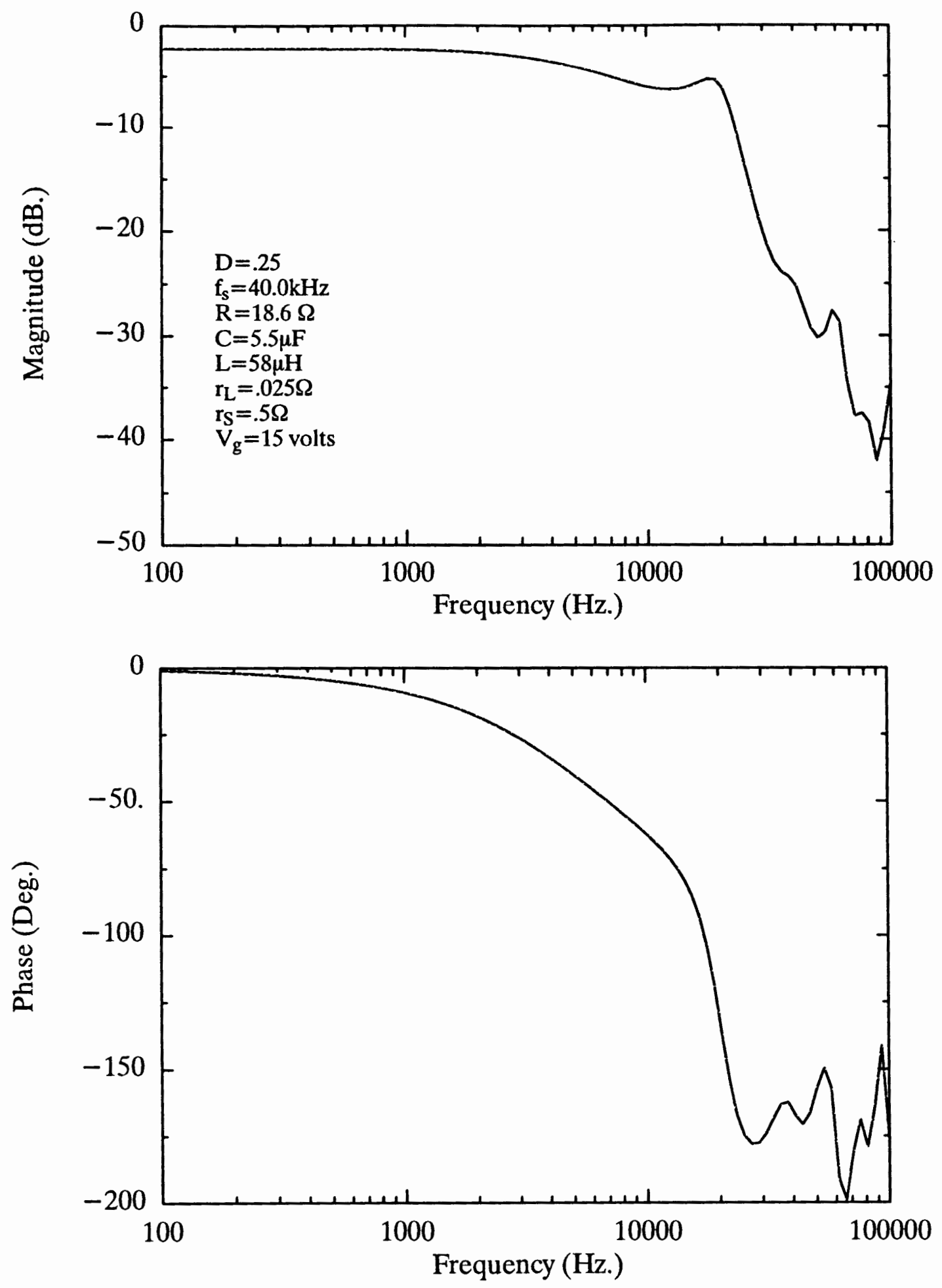

Figure 4- 4 The input-to-output response of the PWM boost converter operating in CCM under current mode programming; exact (solid line) versus the first model (dashed line). 


\section{CHAPTER 5 \\ SECOND MODEL: NEW METHOD FOR OBTAINING \\ CONTROLLER PARAMETERS}

\subsection{General Analysis of the New Model}

Since the original modeling approach given in the last chapter is not satisfactory, alternative approaches need be investigated to avoid the problem of nulling both states of the control-to-output transfer function.

In section 2.1.4 the boost derived hybrid parameter switch model was introduced. Equations $(2-41)$ and $(2-42)$ which are given below were derived from this model.

$$
\begin{aligned}
& \hat{v}_{c a}=v_{i d} \hat{d}+h_{r} \hat{v}_{p a}+z_{i} \hat{i}_{c} \\
& \hat{i}_{p}=i_{o d} \hat{d}+h_{f} \hat{i}_{c}+y_{o} \hat{v}_{p a}
\end{aligned}
$$

In the above set of equations, there exist six unknown parameters. Four of these parameters, $h_{r}, z_{i}, h_{f}$, and $y_{o}$, are found using the input-to-output transfer function. The other two parameters, $v_{i d}$ and $i_{o d}$, are found by employing the control-to-output transfer function.

In equation $(2-41)$ there are two known parameters, $h_{r}$ and $z_{i}$, and one unknown parameter, $v_{i d}$. Therefore, one can solve for the unknown. In this case, the parameter $v_{i d}$ can be evaluated by dividing the variables in equation $(2-41)$ by $\hat{d}$ :

$$
\frac{\hat{v}_{c a}}{\hat{d}}=v_{i d}+h_{r} \frac{\hat{v}_{p a}}{\hat{d}}+z_{i} \frac{\hat{i}_{c}}{\hat{d}}
$$


Rearrangement will give:

$$
v_{i d}=\frac{\hat{v}_{c a}}{\hat{d}}-h_{r} \frac{\hat{v}_{p a}}{\hat{d}}-z_{i} \frac{\hat{i}_{c}}{\hat{d}}
$$

and $i_{o d}$ is similarly found by applying the same technique to equation $(2-42)$.

$$
i_{\text {od }}=\frac{\hat{i_{p}}}{\hat{d}}-y_{o} \frac{\hat{v}_{p a}}{\hat{d}}-h_{f} \frac{\hat{i_{c}}}{\hat{d}}
$$

Equations $(5-1)$ and $(5-2)$ can be given in the following form:

$$
\begin{aligned}
& v_{i d}=v_{i d}^{\prime}-h_{r} \cdot v_{o d}-z_{i} \cdot i_{i d} \\
& i_{o d}=i_{o d}{ }^{\prime}-y_{o \cdot} \cdot v_{o d}-h_{f} i_{i d}
\end{aligned}
$$

where

$$
\begin{gathered}
v_{i d}{ }^{\prime}=\frac{\hat{v}_{c a}}{\hat{d}} \\
i_{o d^{\prime}}=\frac{\hat{i}_{p}}{\hat{d}} \\
v_{o d}=\frac{\hat{v}_{p a}}{\hat{d}} \\
i_{i d}=\frac{\hat{i_{c}}}{\hat{d}}
\end{gathered}
$$

Equations $(5-3)$ and $(5-4)$ are expanded below to show the existing constraints on each parameter:

$$
v_{i d}=\frac{\hat{v}_{c a}}{\hat{d}}-\frac{\hat{v}_{c a}}{\hat{v}_{p a}}\left|\begin{array}{l}
\hat{d}=0 \\
\hat{i}_{c}=0
\end{array} \cdot \frac{\hat{v}_{p a}}{\hat{d}}-\frac{\hat{v}_{c a}}{\hat{i}_{c}}\right| \begin{aligned}
& \hat{d}=0 \\
& \hat{v}_{p a}=0
\end{aligned} \cdot \frac{\hat{i_{c}}}{\hat{\lambda}}
$$




$$
i_{o d}=\frac{\hat{i_{p}}}{\hat{d}}-\left.\frac{\hat{i}_{p}}{\hat{v}_{p a}}\right|_{\hat{i}=0} ^{\hat{d}=0} \cdot \frac{\hat{v}_{p a}}{\hat{d}}-\frac{\hat{i}_{p}}{\hat{i}_{c}} \mid \begin{gathered}
\hat{d}=0 \\
\hat{v}_{p a}=0
\end{gathered} \cdot \frac{\hat{i_{c}}}{\hat{d}}
$$

Therefore, this method will be valid only when the nulling according to equations $(5-9)$ and $(5-10)$ is performed.

\subsubsection{PWM Duty Ratio Programmed Model}

In this section, the equivalent boost model given in Figure $2-7$ is simulated using the second hybrid parameter switch model. The state equation for this model is the same as equation $(4-8)$. Then, the control-to-output and input-to-output frequency responses of the second modeling approach are compared against the exact results.

As shown in Figure 5 - 1 the small - signal control - to-output frequency response of the PWM boost hybrid parameter switch model matches that of the exact small-signal control-to-output transfer function given in equation $(3-43)$. The input-tooutput response of the above model is shown in Figure $5-2$. Also, the exact smallsignal input-to-output transfer function $(3-44)$ is plotted on the same graph. The agreement is excellent. 

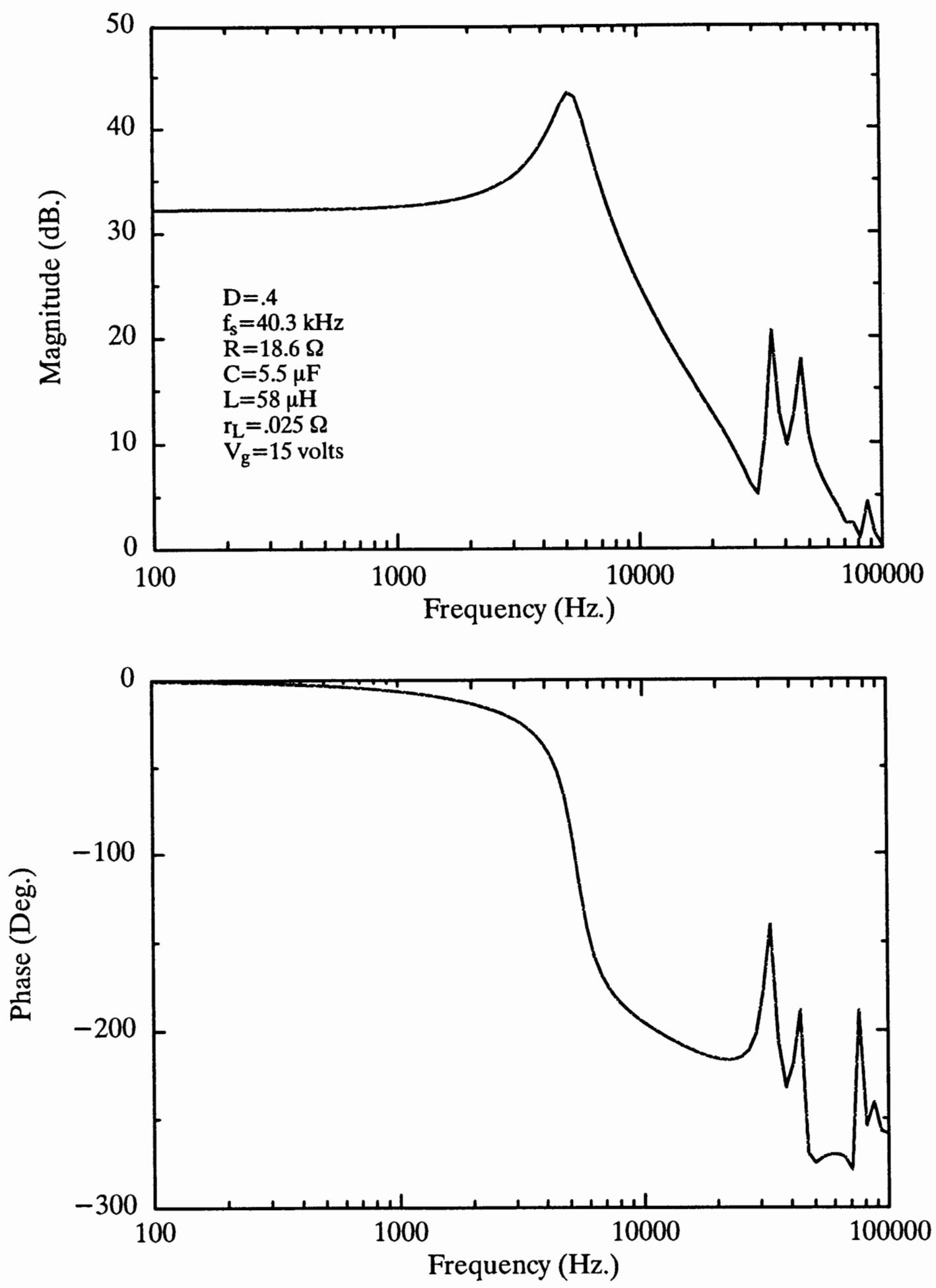

Figure 5 - 1 The control-to-output response of the PWM boost converter operating in CCM; exact (solid line) versus the second model (dashed line). 

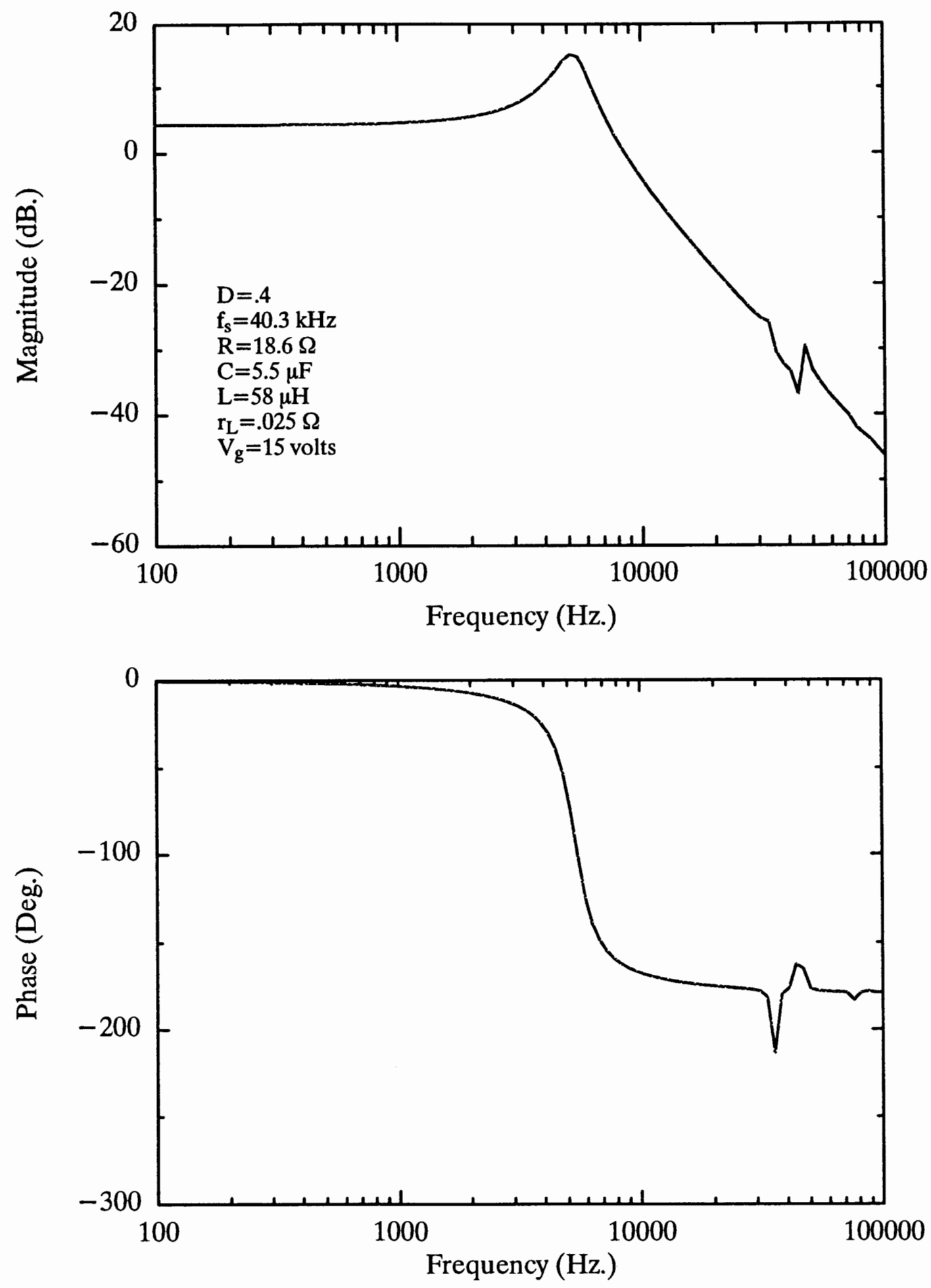

Figure 5-2 The input-to-output response of the PWM boost converter operating in CCM; exact (solid line) versus the second model (dashed line). 
The next step is to obtain the expressions for the switch parameters. The goal is to find simple expressions. The solutions of equations $(3-45),(3-46)$, and $(3-47)$ all will be in exponential matrix form. Therefore, using the exact small-signal transfer functions of $(3-43)$ and $(3-44)$ will result in complex, lengthy expressions that are considered to be in an undesired form. As a result, we make the following assumption:

In a well designed converter, the output capacitor voltage ripple will be small; therefore, the output capacitor can be modeled by a constant voltage source as shown in Figure $5-3$.

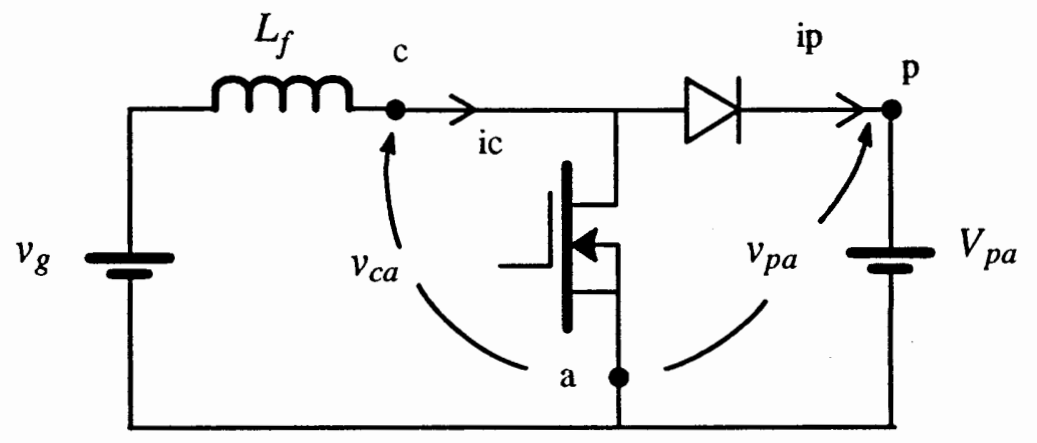

Figure $5-3$ Boost converter: capacitor is replaced by a constant voltage source $V_{p a}$.

This approximation will reduce the number of states to one, i.e. the inductor current state, and consequently equations $(3-43)$ and $(3-44)$ will become scalar. In order to obtain the parameter expressions, the reduced order converter of Figure $5-3$ must be analyzed.

Figure $5-4$ shows the reduced order boost converter in $D T_{s}$ and $D^{\prime} T_{s}$ subintervals. 

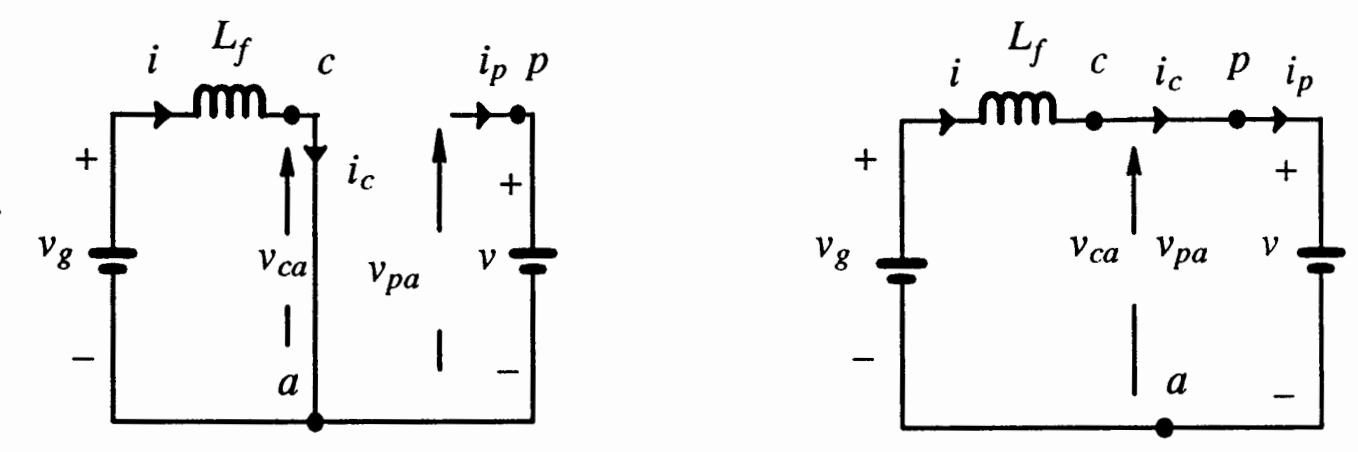

Figure 5 - 4 Reduced order boost converter of Figure $5-3$ in; a) $D T_{s}$ subinterval b) $D^{\prime} T_{s}$ subinterval.

The state vector is now reduced to a single state:

$$
x=i
$$

and the capacitor voltage is considered to be the second input, hence

$$
u=\left[\begin{array}{l}
v_{g} \\
v
\end{array}\right]
$$

$i, v_{g}$, and $v$ contain both the steady-state values and the perturbation quantities.

$$
\begin{aligned}
& i=I+\hat{i} \\
& v_{g}=V_{g}+\hat{v}_{g} \\
& v=V+\hat{v}
\end{aligned}
$$

The steady state solution of Figure $5-4$ can be easily calculated. State equation of the system for $D T_{s}$ subinterval is:

$$
-V_{g}+\frac{d i}{d t} L_{f}=0
$$

and for $D^{\prime} T_{s}$ subinterval is:

$$
-V_{g}+\frac{d i}{d t} L_{f}+V=0
$$


From equations $(5-14)$ and $(5-15)$, the reduced boost converter is described by:

$$
\begin{array}{rlrl}
A_{1} & =0 & A_{2} & =0 \\
B_{1}=\left[\begin{array}{ll}
\frac{1}{L_{f}} & 0
\end{array}\right] & B_{2}=\left[\frac{1}{L_{f}}-\frac{1}{L_{f}}\right]
\end{array}
$$

The output and the transmission matrices for the reduced model are given below: When $y=\hat{v}_{c a}$

$$
\begin{array}{ll}
C_{1}=0 & E_{1}=\left[\begin{array}{ll}
0 & 0
\end{array}\right] \\
C_{2}=0 & E_{2}=\left[\begin{array}{ll}
0 & 1
\end{array}\right]
\end{array}
$$

When $y=\hat{i}_{p}$

$$
\begin{array}{ll}
C_{1}=0 & E_{1}=\left[\begin{array}{ll}
0 & 0
\end{array}\right] \\
C_{2}=1 & E_{2}=\left[\begin{array}{ll}
0 & 0
\end{array}\right]
\end{array}
$$

When $y=\hat{i}_{c}$

$$
\begin{array}{ll}
C_{1}=1 & E_{1}=\left[\begin{array}{ll}
0 & 0
\end{array}\right] \\
C_{2}=1 & E_{2}=\left[\begin{array}{ll}
0 & 0
\end{array}\right]
\end{array}
$$

The reduction in the number of states will effect the overall frequency characteristics of the model.

To obtain the numeric solutions for the parameters of the reduced order model, two converter systems are set up simultaneously. The first system is the reduced order system. The state and output matrices for this system are passed on to appropriate functions to obtain parameter values. The second system is the full order system used in the exact small-signal analysis method. The DC operating points of this system are passed to the functions along with the reduced order system's state and output matrices. The returned value of the function calls are the discrete values for the parameters. 
After performing a frequency sweep using the above algorithm, the state and output matrices of the model are obtained. Next, the above findings are compared against the exact results by plotting their control-to-output and input-to-output responses.

The control-to-output responses of the reduced order model are plotted in Figure $5-5$. The magnitude plot shows that the DC gain of the model is by about 0.5 $\mathrm{dB}$. lower than the exact results. Otherwise, the control-to-output magnitude and phase responses are excellent up to half the switching frequency, $f_{s} / 2$.

The input-to-output magnitude and phase responses of the reduced model are shown in Figure 5-6. These input-to-output results are accurate everywhere except at $f_{s}$.

NOTE: The high inductor current ripple contributes to the inaccuracies occurring at switching frequency and its higher harmonics. It can be shown that reducing the inductor ripple improves the accuracy of the model. 

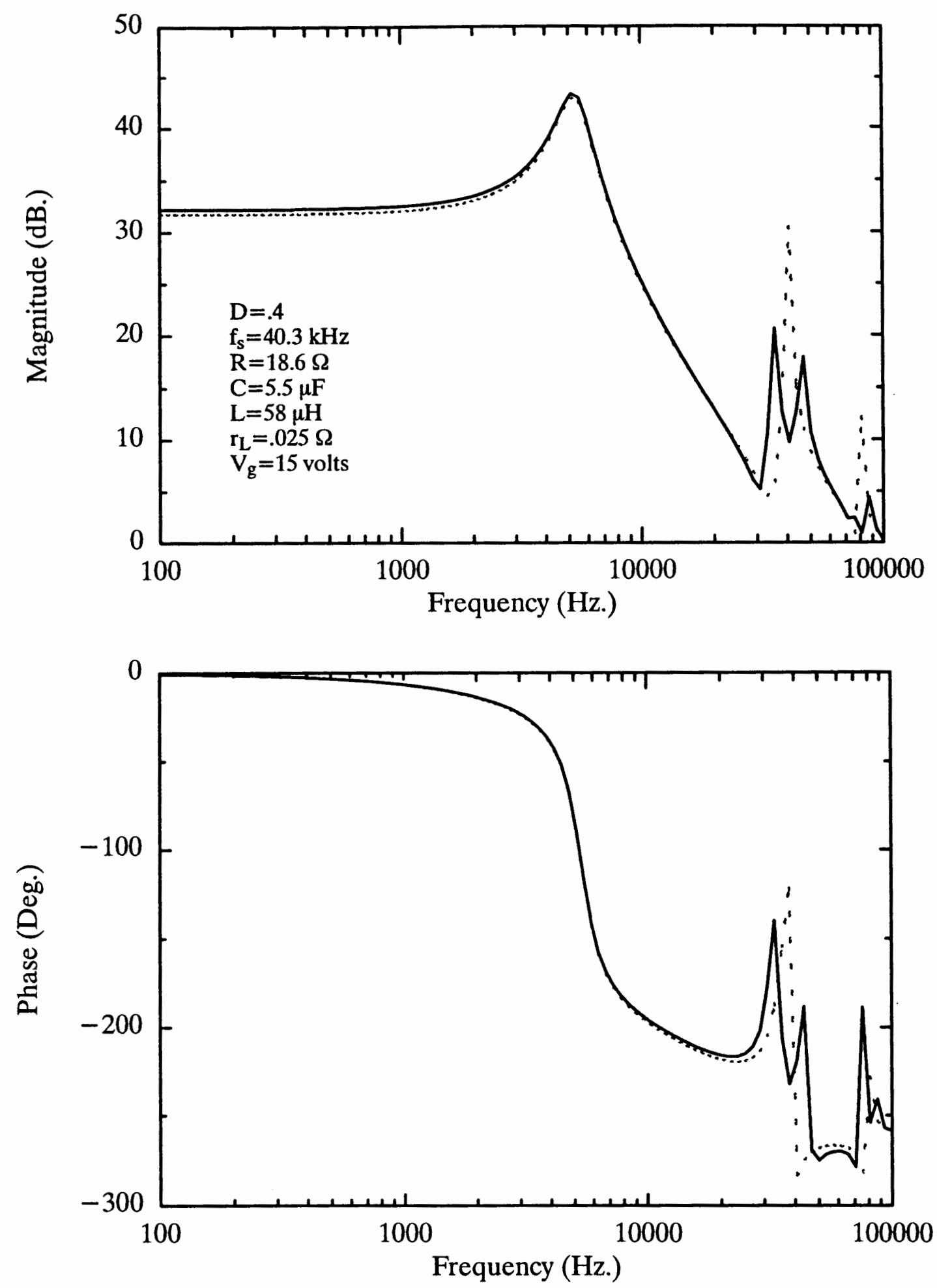

Figure 5- 5 The control-to-output response of the PWM boost converter operating in CCM; exact (solid line) versus the reduced order second model (dashed line). 

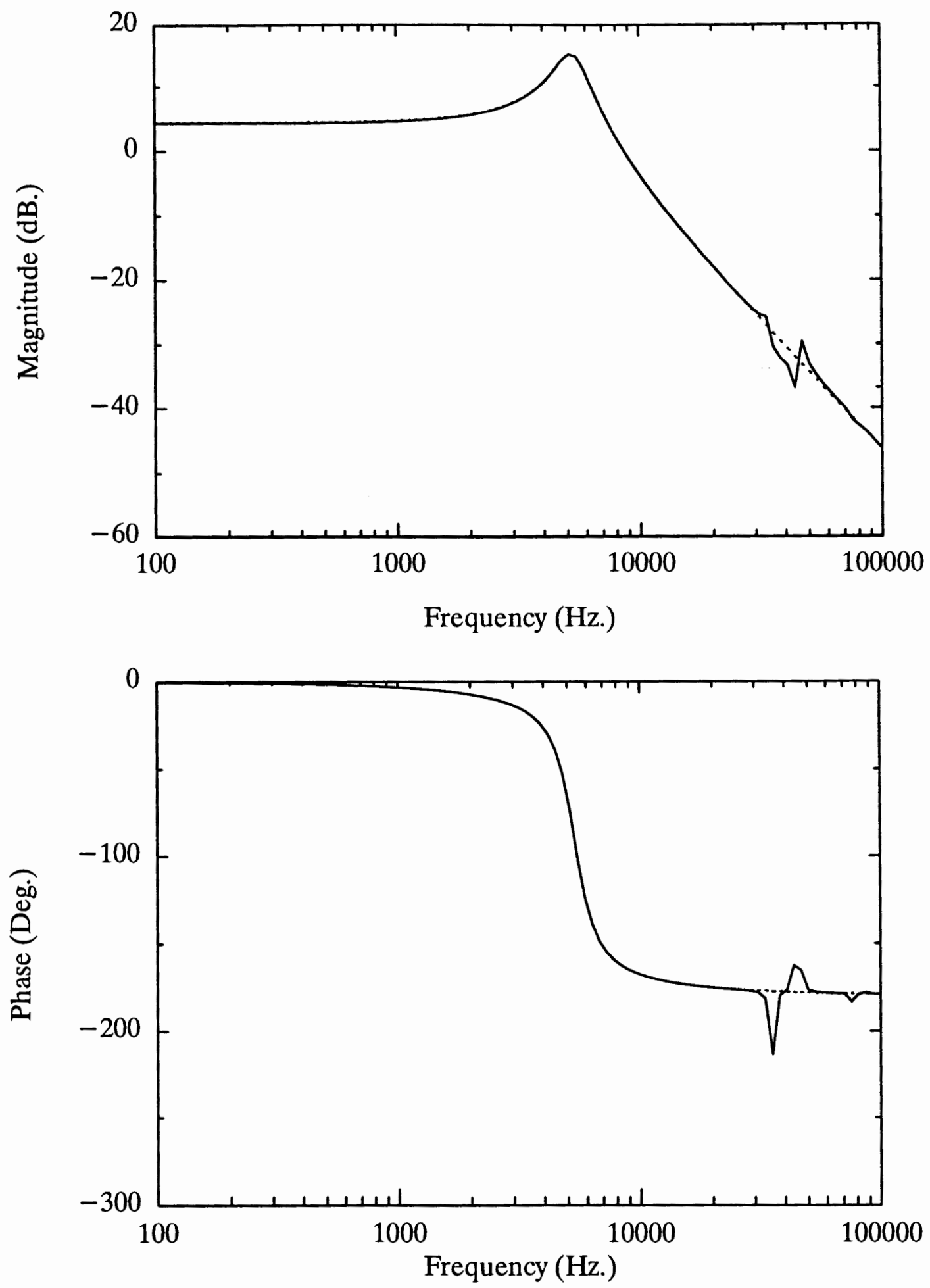

Figure 5- 6 The input - to-output response of the PWM boost converter operating in CCM; exact (solid line) versus the reduced second model (dashed line). 
Maple V has been used to obtain expressions for the proposed reduced model. Since solving these expressions will take much time, only the following parameters are listed:

$$
\begin{aligned}
& v_{i d^{\prime}}=-V_{p a} \\
& h_{r}=D^{\prime} \\
& z_{i}=0 \\
& i_{o d}{ }^{\prime}=\frac{-j V_{p a}\left(1-e^{-j \omega D^{\prime} T_{s}}\right)-I_{c} \omega L\left(1-e^{-j \omega T_{s}}\right)}{\omega L\left(1-e^{-j \omega T_{s}}\right)} \\
& h_{f}=D^{\prime} \\
& y_{o}=0 \\
& i_{i d}=\frac{-j V_{p a}}{\omega L} \\
& v_{o d}=0
\end{aligned}
$$

Substituting the above results in equations $(5-3)$ and $(5-4)$ will give:

$$
v_{i d}=-V_{p a}
$$

and

$$
i_{o d}=\frac{-j V_{p a}\left(1-e^{\left.-j \omega D^{\prime} T_{s}\right)}-I_{c} \omega L\left(1-e^{\left.-j \omega T_{s}\right)}\right.\right.}{\omega L\left(1-e^{-j \omega T_{s}}\right)}+\frac{j D^{\prime} V_{p a}}{\omega L}
$$

For the exact small-signal transfer functions, the instantaneous inductor current and capacitor voltage are used. The model parameters have expressions in terms of $I_{c}$ and $V_{p a}$ which are steady state values. In order for the model to produce frequency responses comparable to the exact results, the instantaneous values must be used. For a boost converter, according to equations $(4-1)$ and $(4-2), I_{c}$ and $V_{p a}$ are equal to the states of the system. The instantaneous quantities can be given by:

$$
I_{c \Delta}=I_{c}+\frac{\Delta i_{c}}{2}
$$


and

$$
V_{p a \Delta}=V_{p a}+\frac{\Delta v_{p a}}{2}
$$

Since $z_{i}$ and $v_{o d}$ were zero, $v_{i d}$ is unchanged and only the $i_{o d}$ parameter has been effected by this method. Therefore, for the PWM duty ratio converters, the new model reduces to Figure $5-7$.

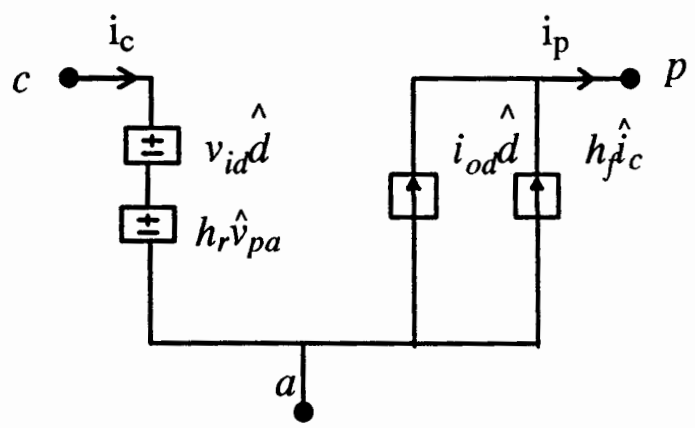

Figure 5 - 7 Hybrid parameter switch model for PWM duty ratio programming.

\subsubsection{PWM Current Programmed Model}

In the previous modeling approach presented in chapter 4 , the control-to-output response of the current programmed model completely failed to predict the exact results. In this section, the current programmed parameters expressions are obtained numerically. Then, they are used to plot the control-to-output and input-to-output responses of the system. Next, these responses are compared against the exact results.

From the control-to-output and input-to-output plots, shown in Figure 5-8 and Figure 5-9, the accuracy of this model can be verified. All the plots match the exact plots. The next step would be to reduce the order of the model to simplify the results. 

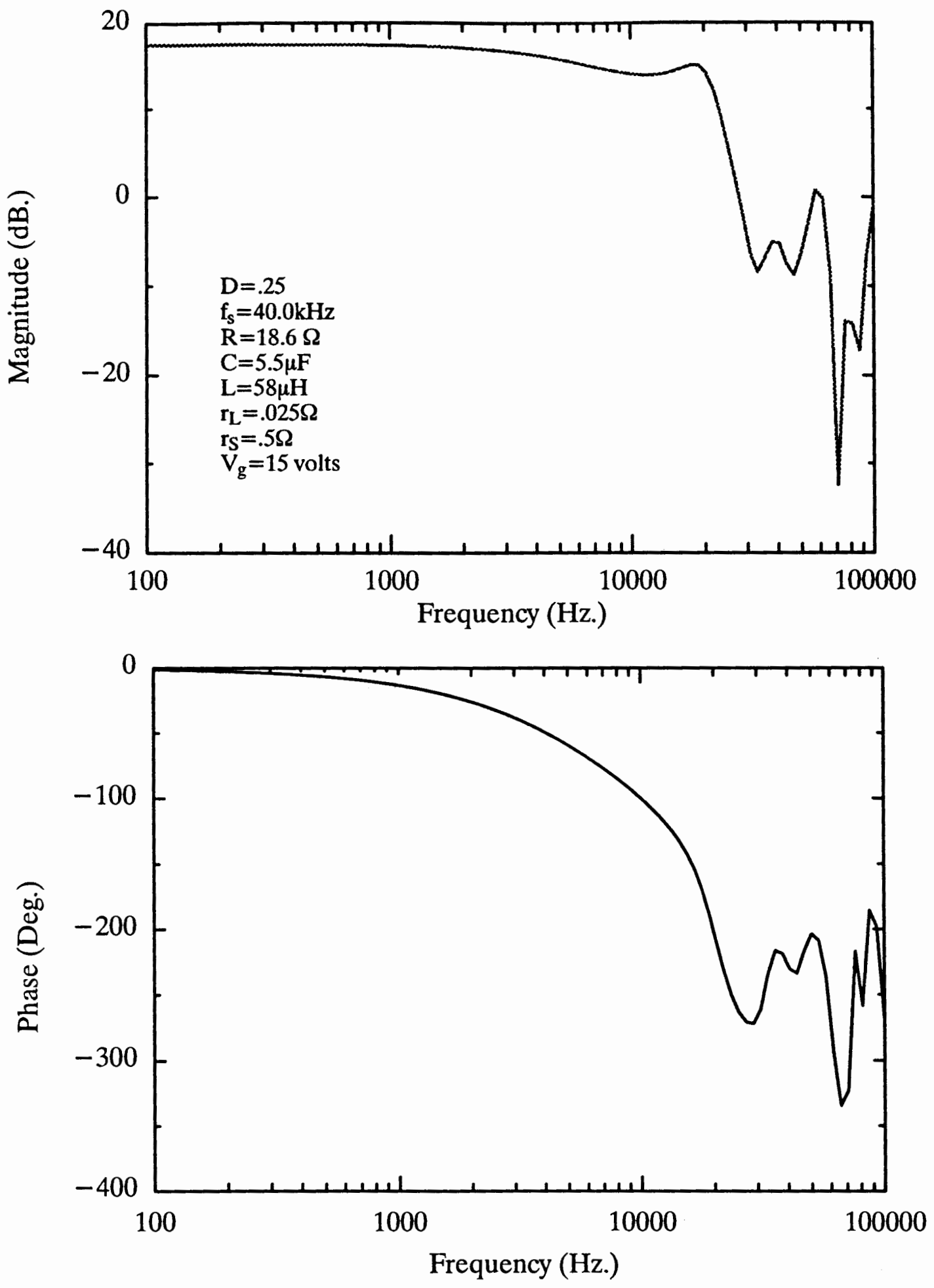

Figure 5-8 The control-to-output response of the PWM boost converter operating in CCM under current mode programming; exact (solid line) versus the second model (dashed line). 

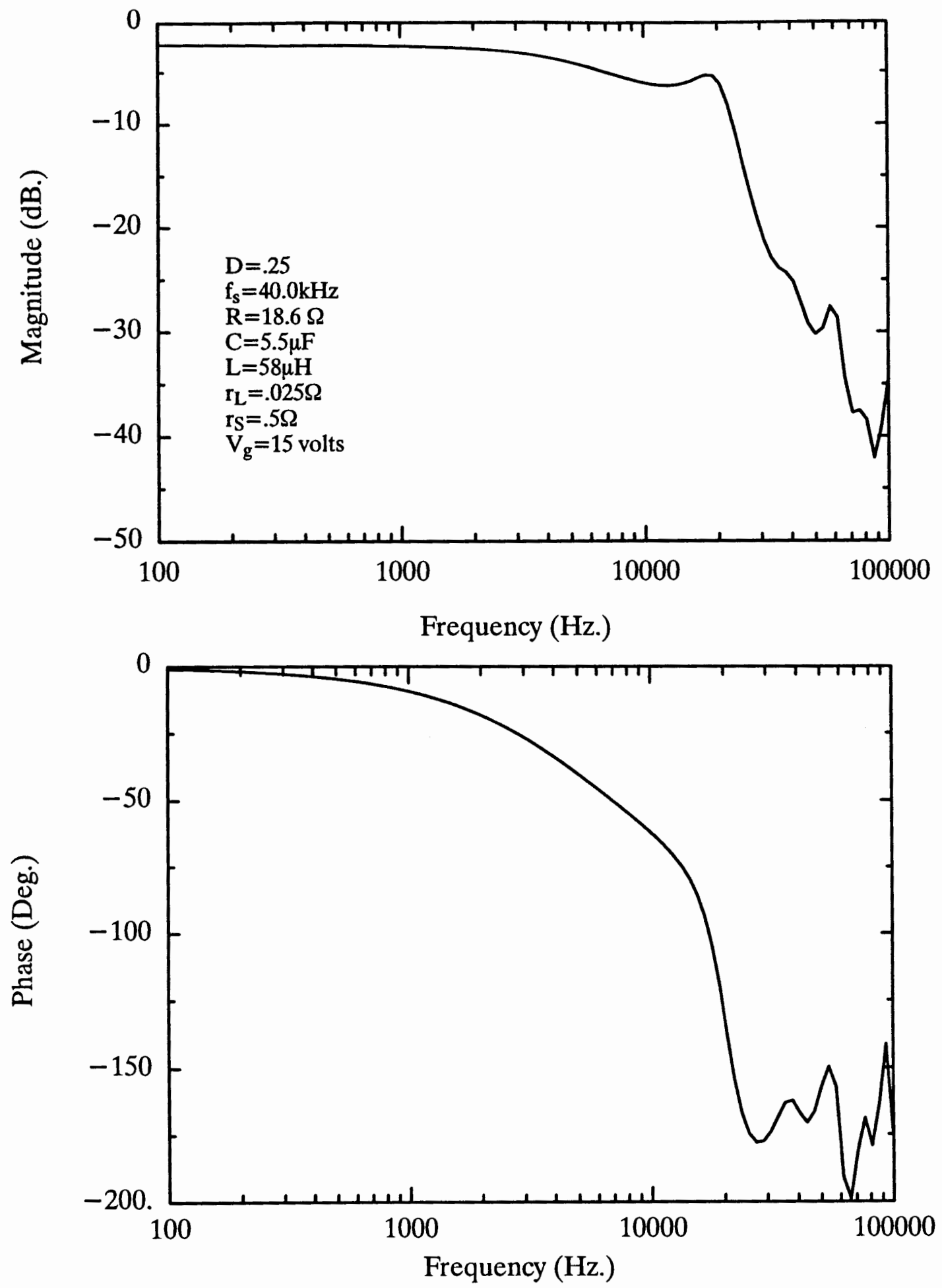

Figure 5 - 9 The input-to-output response of the PWM boost converter operating in CCM under current mode programming ; exact (solid line) versus the second model (dashed line). 
To obtain current mode parameters, the order of the system has to be reduced using the same technique as the one used in the PWM duty ratio case. It is assumed that the output capacitor voltage ripple is very small; hence it is replaced by a constant voltage source.

The analysis done in earlier is used now to plot the responses of the new model. The magnitude response of the control-to-output transfer function for the case of reduced order current mode model deviates from the exact small-signal transfer function results by around $3 \mathrm{~dB}$ as shown in Figure $5-11$. This is a good result, considering the approximation initially made by using an independent voltage source in place of the capacitor. It should be mentioned that the model is tested on a circuit with high inductor current ripple. The control-to-output phase of the reduced model barely has any phase difference of the exact frequency analysis before reaching $f_{s} / 2$. Figure 5-12 shows the input-to-output frequency response for the current mode boost converter model. The same conclusions can be derived for the magnitude response of the input-to-output as those for the control-to-output response. The phase is shifted by about 5 degrees.

The next step is to derive the symbolic expressions for the parameters. Despite the efforts to reduce the complexity of the expressions, few parameters are found to be lengthy and complex. The expressions for the current mode hybrid parameter switch parameters are listed below.

$$
\begin{aligned}
& v_{i d}^{\prime}=-\frac{V_{p a}\left(1-e^{-j \omega T_{s}}\right)}{\left(1-\Gamma e^{-j \omega T_{s}}\right)} \\
& i_{i d}=\frac{V_{p a}}{j \omega L} \frac{\left(1-e^{-j \omega T_{s}}\right)}{\left(1-\Gamma e^{-j \omega T_{s}}\right)}
\end{aligned}
$$




$$
\begin{aligned}
& i_{o d}{ }^{\prime}=-\left(\frac{I_{c}\left(1-e^{\left.-j \omega T_{s}\right)}\right.}{\left(1-\Gamma e^{-j \omega T_{s}}\right)}+\frac{j V_{p a}\left(1-e^{-j \omega D^{\prime} T_{s}}\right)}{\omega L\left(1-\Gamma e^{-j \omega T_{s}}\right)}\right) \\
& h_{r}=D^{\prime} \\
& h_{f}=\frac{1-j \omega D^{\prime} T s-e^{-j \omega D^{\prime} T s}-\Gamma\left(1-e^{-j \omega D^{\prime} T s}-j \omega D^{\prime} T s e^{-j \omega T s}\right)}{1-\Gamma-j \omega T s-(1-\Gamma-j \omega T s \Gamma) e^{-j \omega T s}} \\
& -\frac{j \omega \mu h^{T} I_{c}\left(1-e^{-j \omega T s}\right)}{1-\Gamma-j \omega T s-(1-\Gamma-j \omega T s \Gamma) e^{-j \omega T s}} \\
& z_{i}=\frac{-j \omega V_{p a} \mu h^{T}\left(1-e^{-j \omega T s}\right)}{1-\Gamma-j \omega T s-(1-\Gamma-j \omega T s \Gamma) e^{-j \omega T s}} \\
& y_{o}=0
\end{aligned}
$$

Next, equations $(5-3)$ and $(5-4)$ will be used to find the new $v_{i d}$ and $i_{o d}$ parameters. Since $v_{o d}=0$, these equations will be reduced to the following;

$$
\begin{aligned}
& v_{i d}=v_{i d}{ }^{\prime}-z_{i} \cdot i_{i d} \\
& i_{o d}=i_{o d}{ }^{\prime}-h_{f} i_{i d}
\end{aligned}
$$

Expanding equations $(5-38)$ and $(5-39)$ lead to complex expressions which are not listed here.

Based on the above parameters and results, the new hybrid parameter switch model for current mode programming is shown in Figure $5-10$.

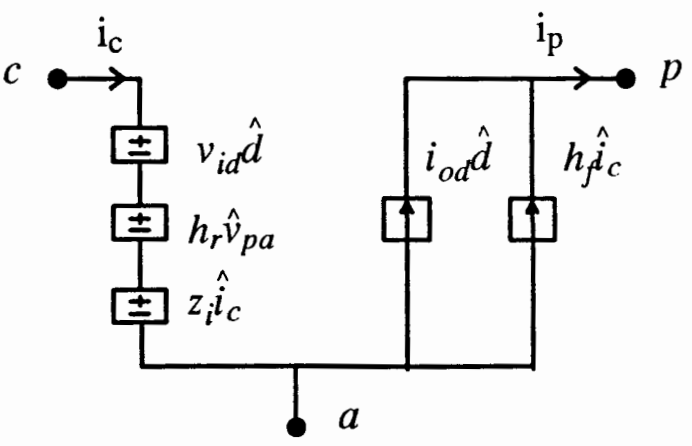

Figure 5 - 10 Hybrid parameter switch model for current mode programming. 

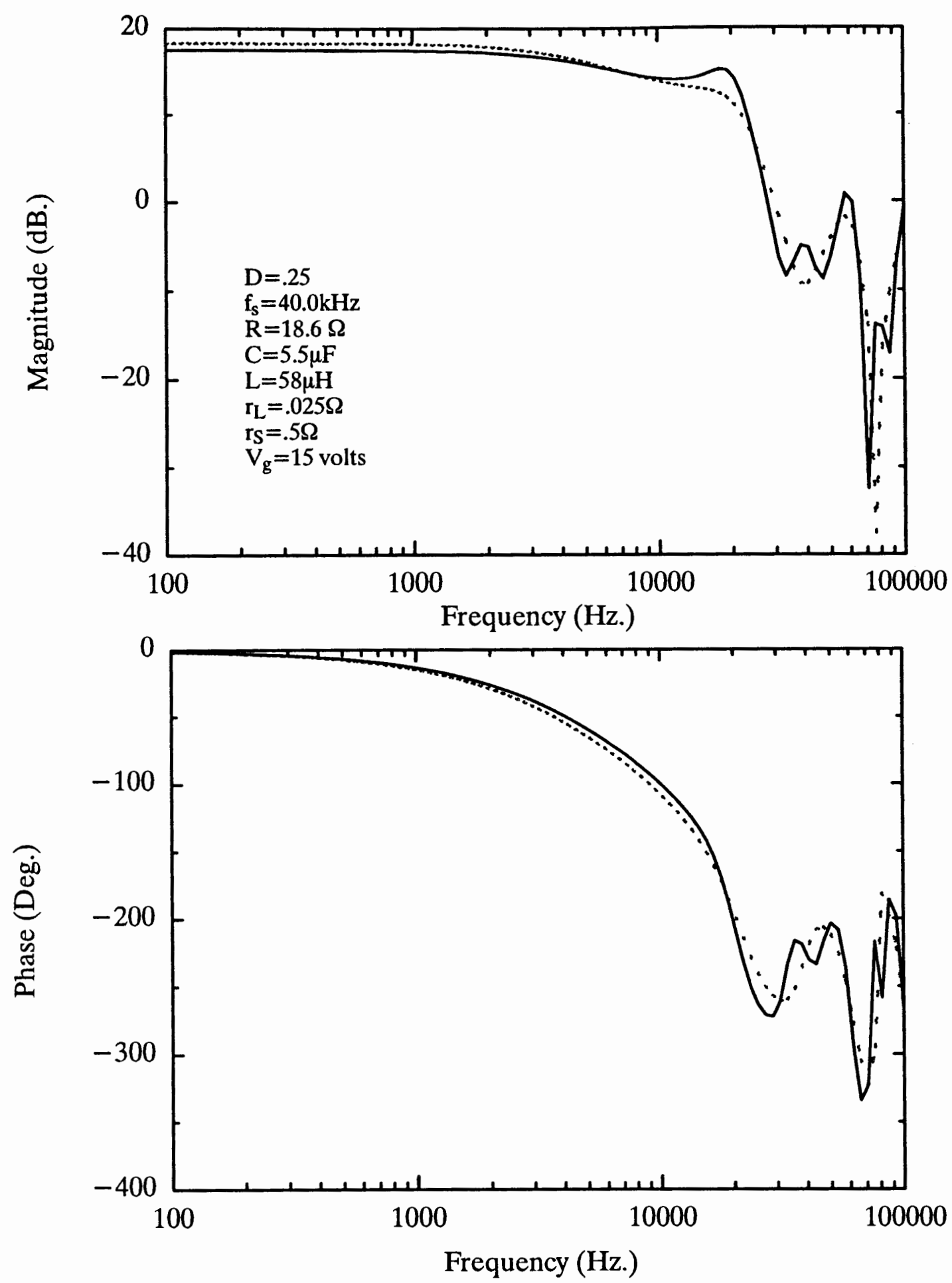

Figure 5-11 The control-to-output response of the PWM boost converter operating in CCM under current mode programming; exact (solid line) versus the reduced order second model (dashed line). 

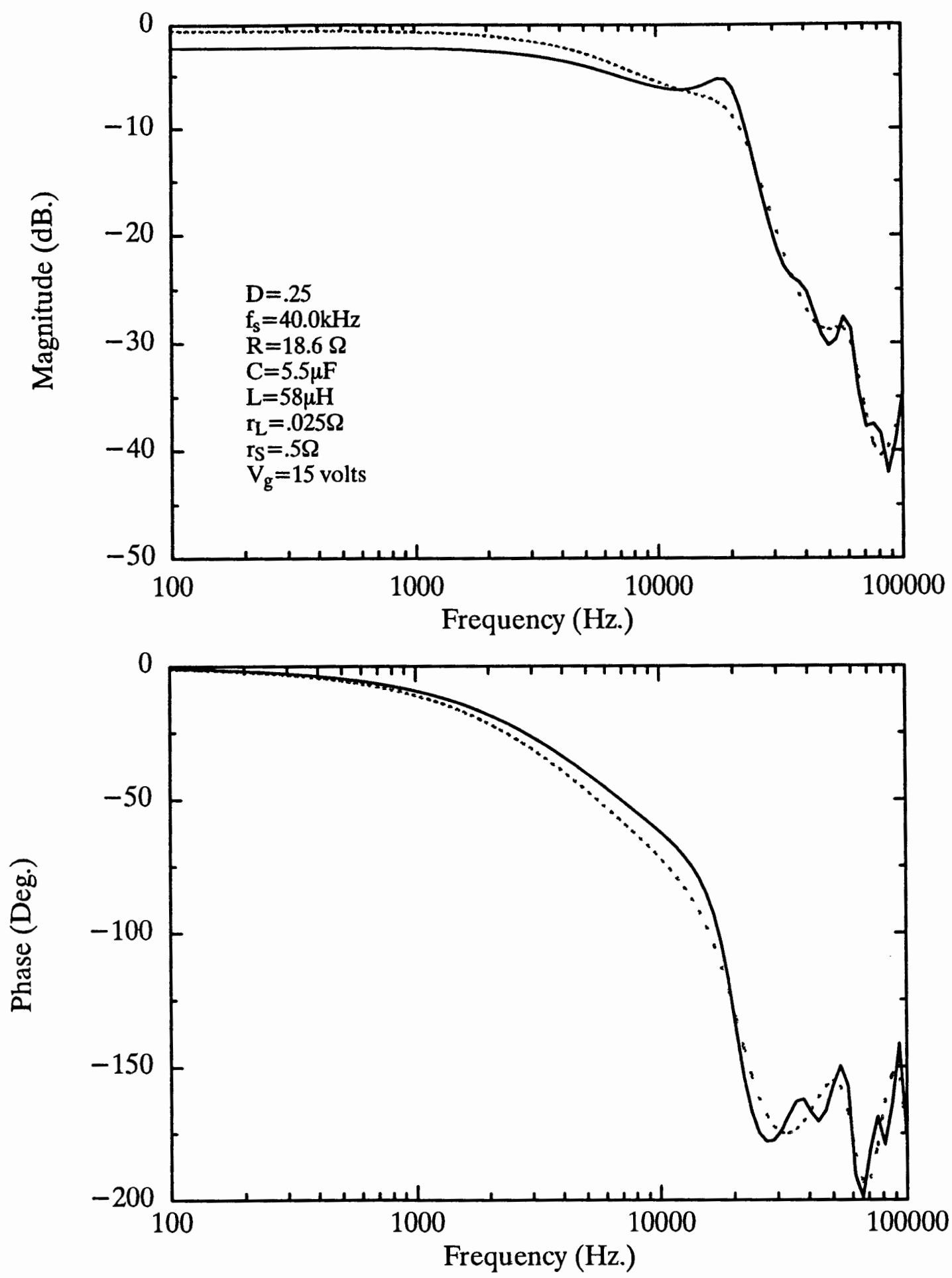

Figure 5-12 The input-to-output response of the PWM boost converter operating in CCM under current mode programming; exact (solid line) versus the reduced order second model (dashed line). 


\subsection{Buck Converter Analysis}

In this section, the accuracy and usefulness of the model will be examined by analyzing the small-signal response of the buck converter. This analysis is conducted on both control schemes; duty-ratio and current-mode programming.

In order for the new, boost derived, hybrid parameter switch model to work with other converters, one must first solve for the dc operating point quantities; $V_{p a}$, and $I_{c}$. This is necessary because the model parameters have expressions that in someway contain the above dc terms. For example, equation ( 5 - 27) gives:

$v_{i d}=-V_{p a}$

and from $(5-28)$ it is known that

$i_{o d}=\frac{-j V_{p a}\left(1-e^{\left.-j \omega D^{\prime} T_{s}\right)}-I_{c} \omega L\left(1-e^{\left.-j \omega T_{s}\right)}\right.\right.}{\omega L\left(1-e^{-j \omega T_{s}}\right)}+\frac{j D^{\prime} V_{p a}}{\omega L}$

\subsubsection{PWM Duty Ratio Programmed Buck Converter}

A schematic of a basic PWM buck converter is shown in Figure 5-13. The orientation of the internal switch devices is not important for small-signal analysis. It is important, however, to appropriately indicate the direction of the independent voltage source $\left(v_{p a}\right)$ and the current source $\left(i_{c}\right)$ as shown in Figure $5-13$.

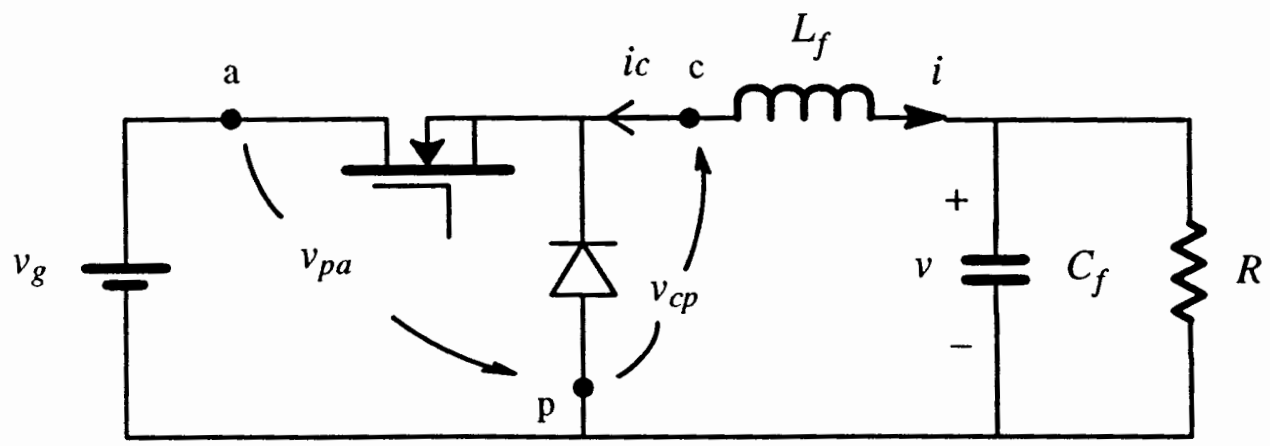

Figure 5 - 13 Circuit schematics for PWM buck converter. 
Inspection of Figure $5-13$ shows that

$$
\begin{aligned}
& v_{p a}=-v_{g} \\
& i_{c}=-i
\end{aligned}
$$

The above set of equations contain both steady-state values and perturbation quantities. Therefore, both sides of above equations can be written in the following manner:

$$
\begin{aligned}
& \hat{v}_{p a}+V_{p a}=-\left(\hat{v}_{g}+V_{g}\right) \\
& \hat{i}_{c}+I_{c}=-(\hat{i}+I)
\end{aligned}
$$

First the dc quantities $I_{c}$ and $V_{p a}$ of the buck converter of Figure $5-13$ need be solved. Separating the steady state term of equations $(5-42)$ and $(5-43)$ will give:

$$
\begin{aligned}
& V_{p a}=-V_{g} \\
& I_{c}=-I
\end{aligned}
$$

The small-signal terms are:

$$
\begin{aligned}
& \hat{v}_{p a}=-\hat{v}_{g} \\
& \hat{i}_{c}=-\hat{i}
\end{aligned}
$$

From Figure $5-13$ the following dc operating point is found:

$$
I=\frac{V_{g} D}{R}
$$

Substituting $(5-48)$ into $(5-45)$ will give:

$$
I_{c}=-\frac{V_{g} D}{R}
$$

The equivalent small-signal circuit model of the buck converter of Figure $5-13$ is depicted in Figure $5-14$. 


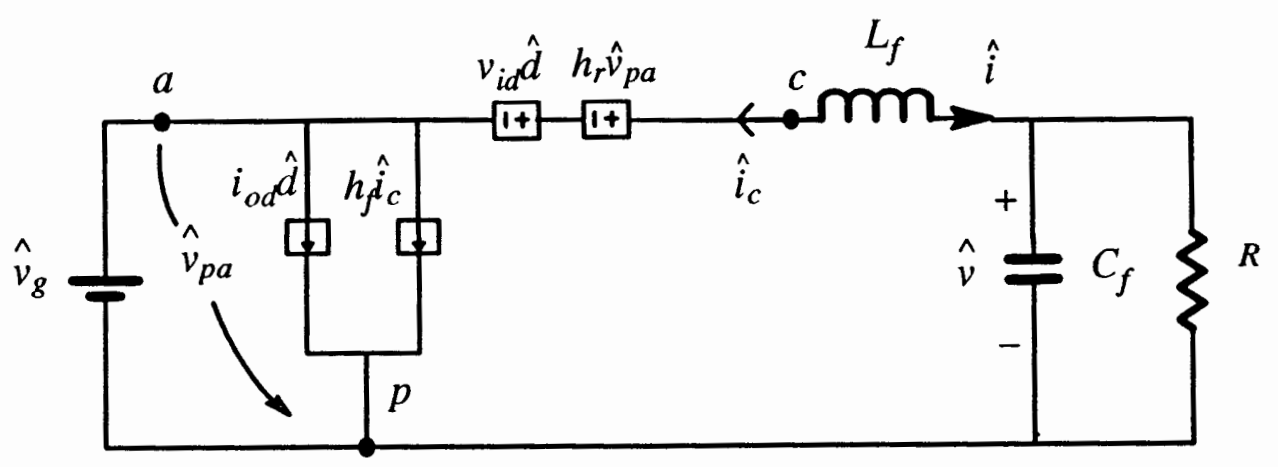

Figure 5 - 14 Hybrid parameter switch model for small-signal analysis of the buck converter of Figure $5-13$.

To determine the control-to-output transfer function, $\hat{v}_{g}$ will be shorted and Figure 5- 14 will reduce to Figure 5 - 15:

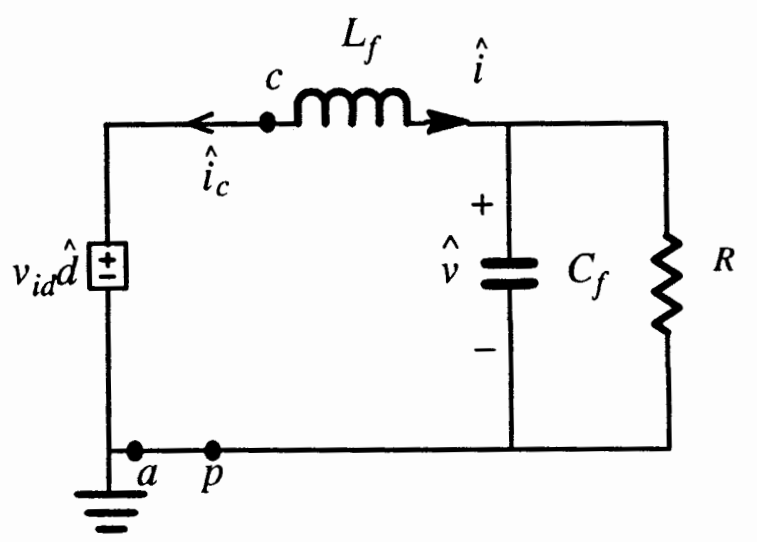

Figure 5-15 Small-signal control-to-output equivalent model of the buck converter of Figure 5 - 13.

Using KVL:

$$
-v_{i d} \hat{d}+\frac{\hat{d i}}{d t} L_{f}+\hat{v}=0
$$

and from KCL, one gets:

$$
\hat{i}-\frac{\hat{d} \hat{v}}{d t} C_{f}-\frac{\hat{v}}{R}=0
$$


Rearranging equations $(5-50)$ and $(5-51)$ will give :

$$
\begin{aligned}
& \frac{\hat{d i}}{d t}=-\frac{1}{L_{f}} \hat{v}+\frac{v_{i d}}{L_{f}} \hat{d} \\
& \frac{d \hat{v}}{d t}=\frac{1}{C_{f}} \hat{i}-\frac{1}{R C_{f}} \hat{v}
\end{aligned}
$$

From equations $(5-52)$ and $(5-53)$ the control - to-output state equation can be set up:

$$
\frac{d}{d t}\left[\begin{array}{l}
\hat{i} \\
\hat{v}
\end{array}\right]=\left[\begin{array}{cc}
0 & -\frac{1}{L_{f}} \\
\frac{1}{C_{f}} & -\frac{1}{R C_{f}}
\end{array}\right]\left[\begin{array}{c}
\hat{i} \\
\hat{v}
\end{array}\right]+\left[\begin{array}{c}
\frac{v_{i d}}{L_{f}} \\
0
\end{array}\right] \hat{d}
$$

Next, the input-to-output equivalent model of Figure $5-14$ is obtained by deactivating the controller terms. This model is shown in Figure 5-16.

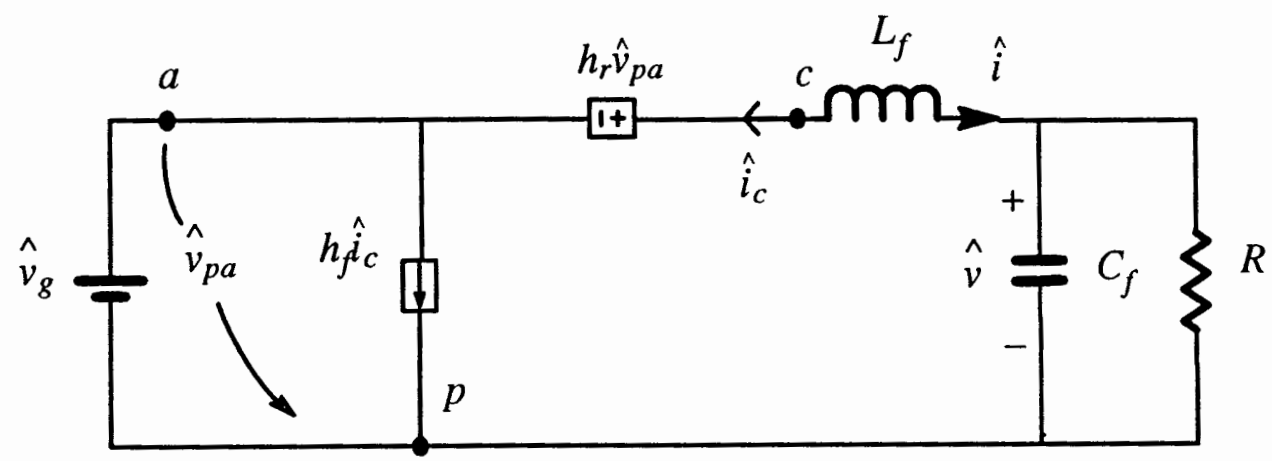

Figure 5-16 Equivalent input - to-ouput small-signal hybrid parameter switch model for the PWM buck converter of Figure 5-13.

Again using KVL:

$$
-\hat{v}_{g}-h_{r} \hat{v}_{p a}+\frac{\hat{d i}}{d t} L_{f}+\hat{v}=0
$$

and $\mathrm{KCL}$ : 
$\hat{i}-\frac{d \hat{v}}{d t} C_{f}-\frac{\hat{v}}{R}=0$

Using equations $(5-46)$ in $(5-55)$ gives:

$$
-\hat{v}_{g}+h_{r} \hat{v}_{g}+\frac{\hat{d i}}{d t} L_{f}+\hat{v}=0
$$

while rearranging equations $(5-56)$ and $(5-57)$ gives:

$$
\begin{aligned}
& \frac{\hat{d v}}{d t}=\frac{1}{C_{f}} \hat{i}-\frac{1}{R C_{f}} \hat{v} \\
& \frac{d \hat{i}}{d t}=-\frac{1}{L_{f}} \hat{v}+\frac{\left(1-h_{r}\right)}{L_{f}} \hat{v}_{g}
\end{aligned}
$$

Solution of $(5-58)$ and $(5-59)$ may be given in state equation form :

$$
\frac{d}{d t}\left[\begin{array}{l}
\hat{i} \\
\hat{v}
\end{array}\right]=\left[\begin{array}{cc}
0 & -\frac{1}{L_{f}} \\
\frac{1}{C_{f}}-\frac{1}{R C_{f}}
\end{array}\right]\left[\begin{array}{c}
\hat{i} \\
\hat{v}
\end{array}\right]+\left[\begin{array}{c}
\frac{1-h_{r}}{L_{f}} \\
0
\end{array}\right] \hat{v}_{g}
$$

One could use equations $(2-49)$ and $(2-50)$ to find the desired transfer functions. In Figure $5-17$, the control-to-output plot of the PWM buck described by $(5-60)$ is compared with that of the exact small signal results results. The ripple percentages for this system are:

$$
\begin{aligned}
& \frac{\Delta v / 2}{V} \times 100 \approx 12 \% \\
& \frac{\Delta i / 2}{I} \times 100 \approx 69 \%
\end{aligned}
$$

The response of the model completely matches the exact results. The input-to-output response of the above circuit matches the exact results as shown in Figure 5-18. From above results, it can be seen that the proposed model is valid for the case of PWM buck converter. 

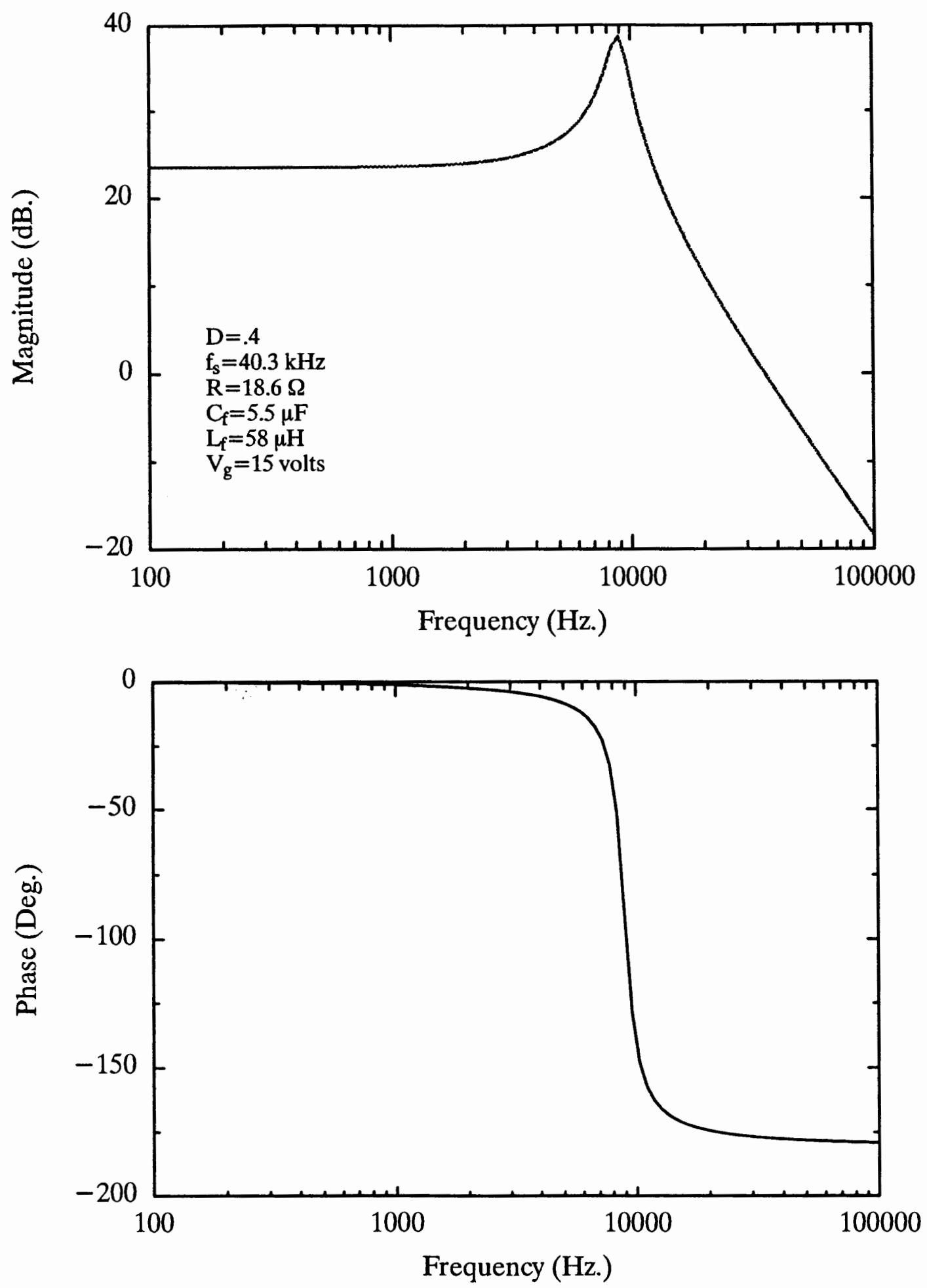

Figure 5-17 The control-to-output response of the PWM buck converter operating in CCM; exact (solid line) versus the second model (dashed line). 

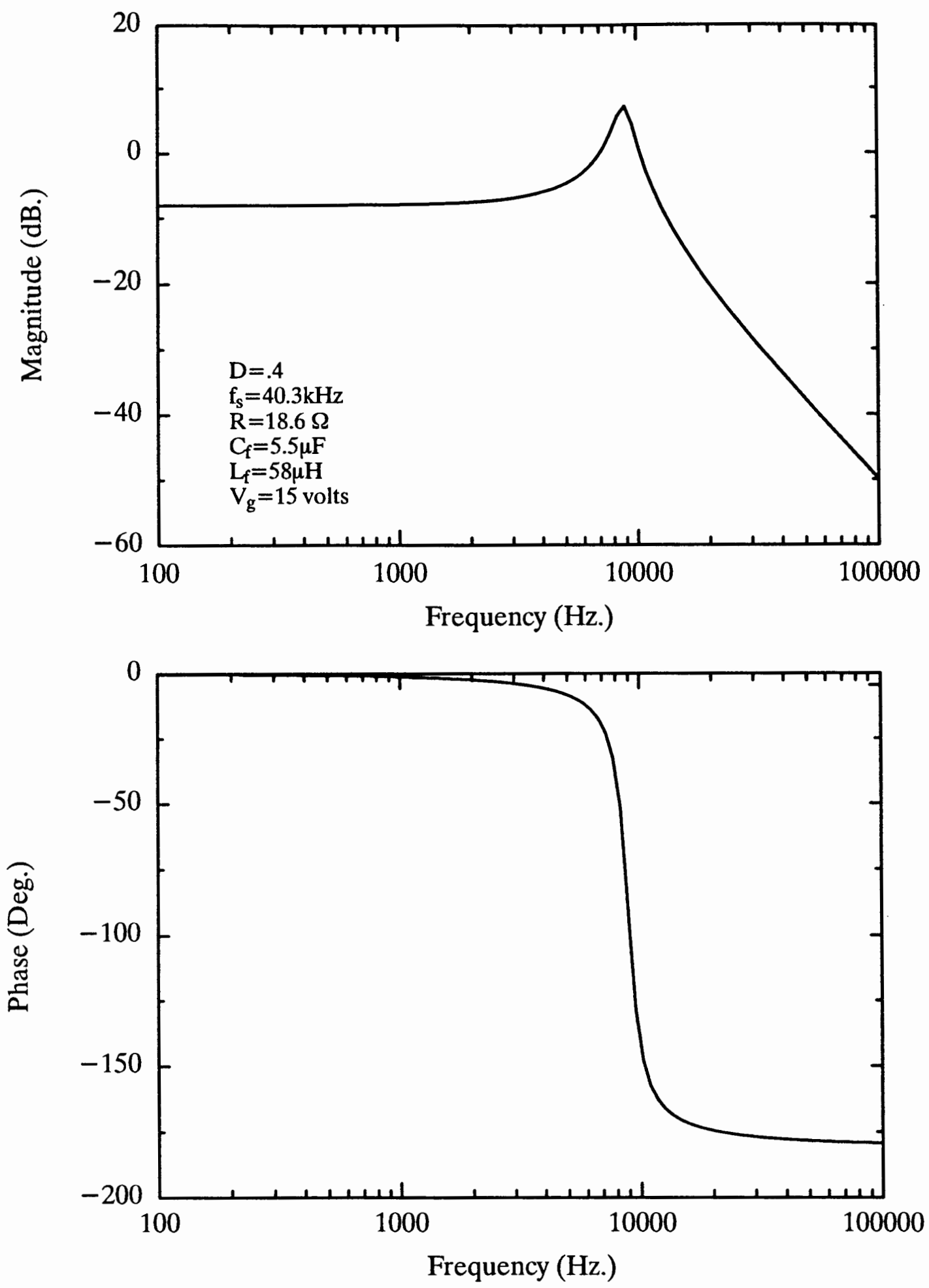

Figure 5-18 The input-to-output response of the PWM buck converter operating in CCM ; exact (solid line) versus the second model (dashed line). 


\subsubsection{PWM Current Programmed Buck Converter}

To verify this modeling approach, the control-to-output and input-to-output responses of the current programmed buck converter of Figure 5-19 is determined. In converter topologies with a single PWM switch implementation, the positive slope of the inductor current is identical to the transistor current slope in the ON position. Therefore, the inductor current is sensed instead of the transistor current.

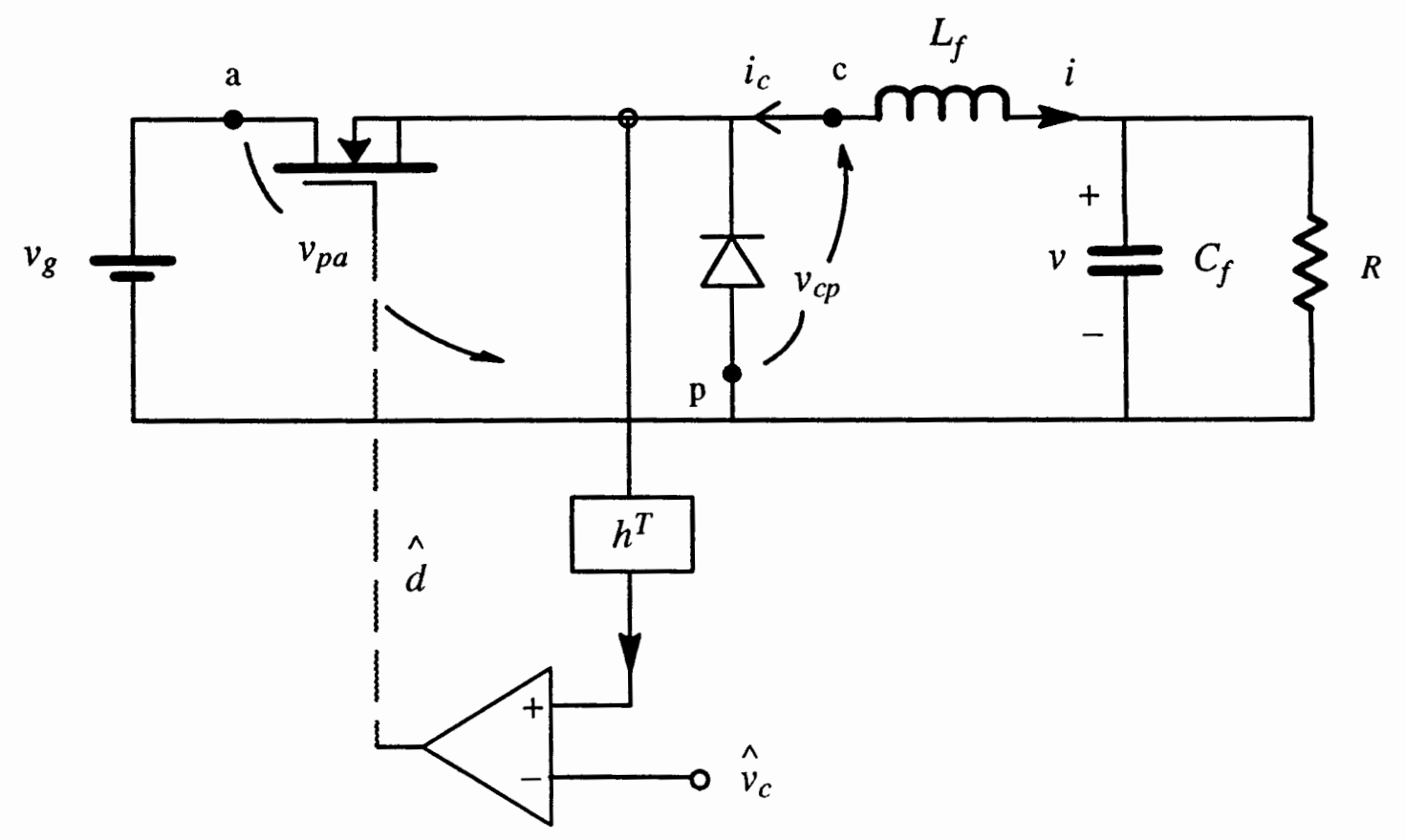

Figure 5-19 Circuit schematics for PWM buck converter.

Using equation $(3-53)$ for a single inductor state gives:

$$
\mu=\frac{1}{h^{T} m_{1}+m_{c}}
$$

where

$h^{T}$ denotes the current to voltage transformation gain, $m_{1}$ is the slope of the rising portion of the sensed current and $m_{c}$ is the compensation ramp. 
Here the inductor current slope is of concern. Therefore, equation $(3-54)$ will reduce to:

$\Gamma=1-\xi h^{T} \mu$

Since $\mu$ and $\Gamma$ are contingent on the slope of the inductor current and stabilizing ramp, they are carried through parameters as symbols. Therefore, depending on the particular converter, $\mu$ and $\Gamma$ will need to be expanded into equations (5-61) and(5-62), respectively, and the results will be incorporated into the parameters expressions.

It is important to change the sign of $h^{T}$ when inductor current $i$ is flowing in the opposite direction of common terminal current $i_{c}$. This is due to the fact that $h^{T}$ is the gain associated with inductor current and must be consistent with the direction of the inductor current flow.

Next, equations $(5-61)$ and $(5-62)$ are solved for the buck converter of Figure $5-19$. The positive slope of the inductor current is :

$$
m_{1}=\frac{V_{g}-V}{L_{f}}
$$

Furthermore, from the dc conversation ratio:

$$
V=V_{g} D
$$

Substituting equation $(5-64)$ into equation $(5-63)$ and simplifying gives :

$$
m_{1}=\frac{V_{g} D^{\prime}}{L_{f}}
$$

Since no compensation ramp has been used in this example, equation $(5-61)$ reduces to :

$$
\mu=\frac{1}{h^{T} m_{1}}
$$

and consequently equation $(5-62)$ reduces to : 


$$
\Gamma=1-\frac{\xi}{m_{1}}
$$

$\xi$ can be found by solving equation $(3-48)$. Therefore,

$$
\xi=\frac{V_{g}}{L_{f}}
$$

Substituting equation $(5-68)$ into equation $(5-67)$ and simplifying will give

$$
\Gamma=-\frac{D}{D^{\prime}}
$$

For the buck converter,

$$
i_{c}=-i
$$

Therefore, the sign of the transformation gain $h^{T}$ must be changed as well, so it would reflect the change in the direction of the inductor current. Hence,

$$
h^{T} \rightarrow-h^{T}
$$

The above dc operating conditions, along with those obtained for the PWM dutyratio programmed buck converter, can be used to deduce the small signal response of the current programmed buck converter of Figure $5-19$. First, the buck converter is modeled using the current programmed hybrid parameter switch model as shown in Figure $5-20$.

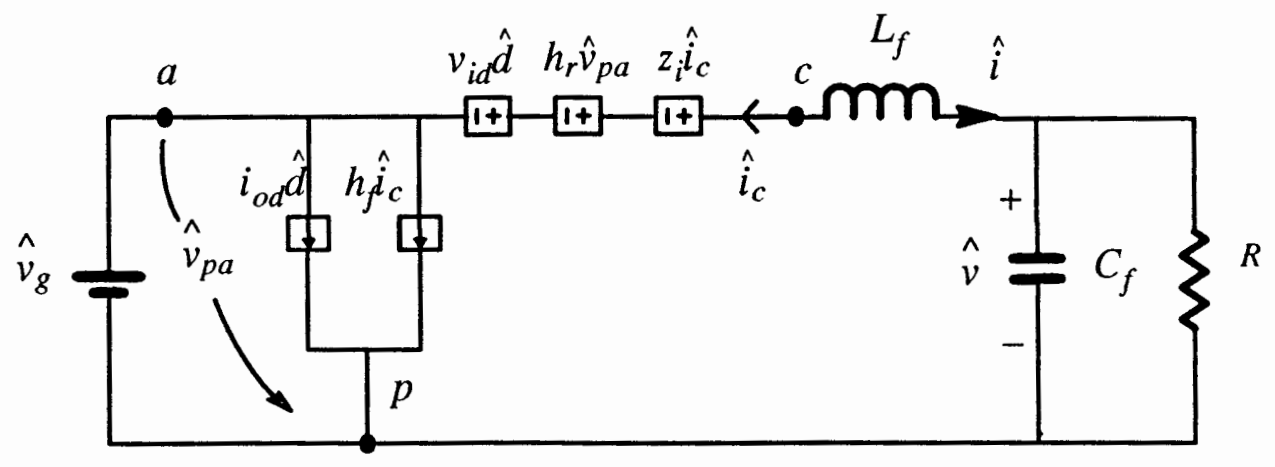

Figure 5-20 Equivalent small-signal hybrid parameter switch model for the PWM buck converter of Figure 5 - 19. 
Analysis of Figure 5-20 is essentially the same as that of the PWM buck converter. The $z_{i}$ parameter needs to be incorporated into the A matrix of equations (5 - 54) and $(5-60)$. The control - to-output and input-to-output equations can be given in the state space representation form as shown in equation $(5-71)$. The controlto-output transfer function can be obtained by deactivating the effect of the line input, $v_{g}$. The input - to-output transfer function can be found in the same manner, by setting the controller quantities to zero. The state space formulation for the current mode buck converter using the hybrid parameter switch model is:

$$
\frac{d}{d t}\left[\begin{array}{l}
\hat{i} \\
\hat{v}
\end{array}\right]=\left[\begin{array}{cc}
-\frac{z_{i}}{L_{f}} & -\frac{1}{L_{f}} \\
\frac{1}{C_{f}} & -\frac{1}{R C_{f}}
\end{array}\right]\left[\begin{array}{l}
\hat{i} \\
\hat{v}
\end{array}\right]+\left[\begin{array}{c}
\frac{1-h_{r}}{L_{f}} \\
0
\end{array}\right] \hat{v}_{g}+\left[\begin{array}{c}
\frac{v_{i d}}{L_{f}} \\
0
\end{array}\right] \hat{d}
$$

Equation $(5-71)$ is used to obtain the frequency response of the model. Only $z_{i}$, $h_{n}$ and $v_{i d}$ parameters are used for the current mode buck analysis. Next, the response of the model is plotted against the exact small signal response of the system. This system has the following ripple percentages:

$$
\begin{aligned}
& \frac{\Delta v / 2}{V} \times 100 \approx 0.16 \% \\
& \frac{\Delta i / 2}{I} \times 100 \approx 12.8 \%
\end{aligned}
$$

The control to output response of this model is accurate everywhere except at the switching frequency and its harmonics as shown in Figure 5-21. As it is apparent from Figure $5-22$, this model fails for the input-to-output response of the current programmed buck converter. The magnitude and the phase response of this model are significantly different from the exact plots. There is an overall $+12 \mathrm{~dB}$. level shift in the magnitude response of the model at low frequencies up to $f_{s} / 2$. The phase plot of the model has about $180^{\circ}$ phase lag. Much of this error is due to the fact that the $h_{r}$ parameter found by this method is equal to that of the PWM duty ratio control and con- 
tains no information about the sensed inductor current. This is further explained in chapter 6. 

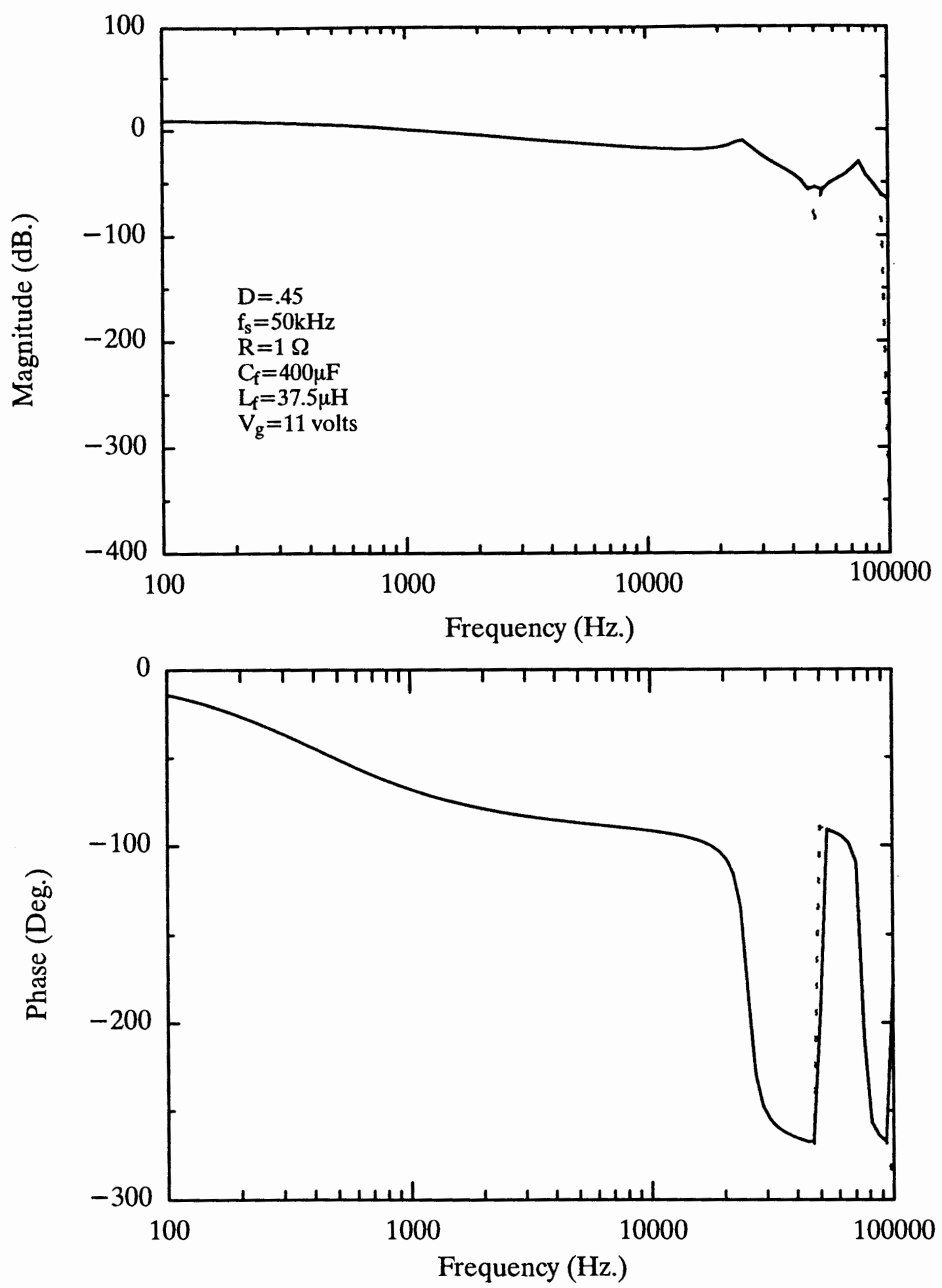

Figure 5-21 The control-to-output response of the PWM buck converter operating in CCM under current mode programming; exact (solid line) versus the second model (dashed line). 

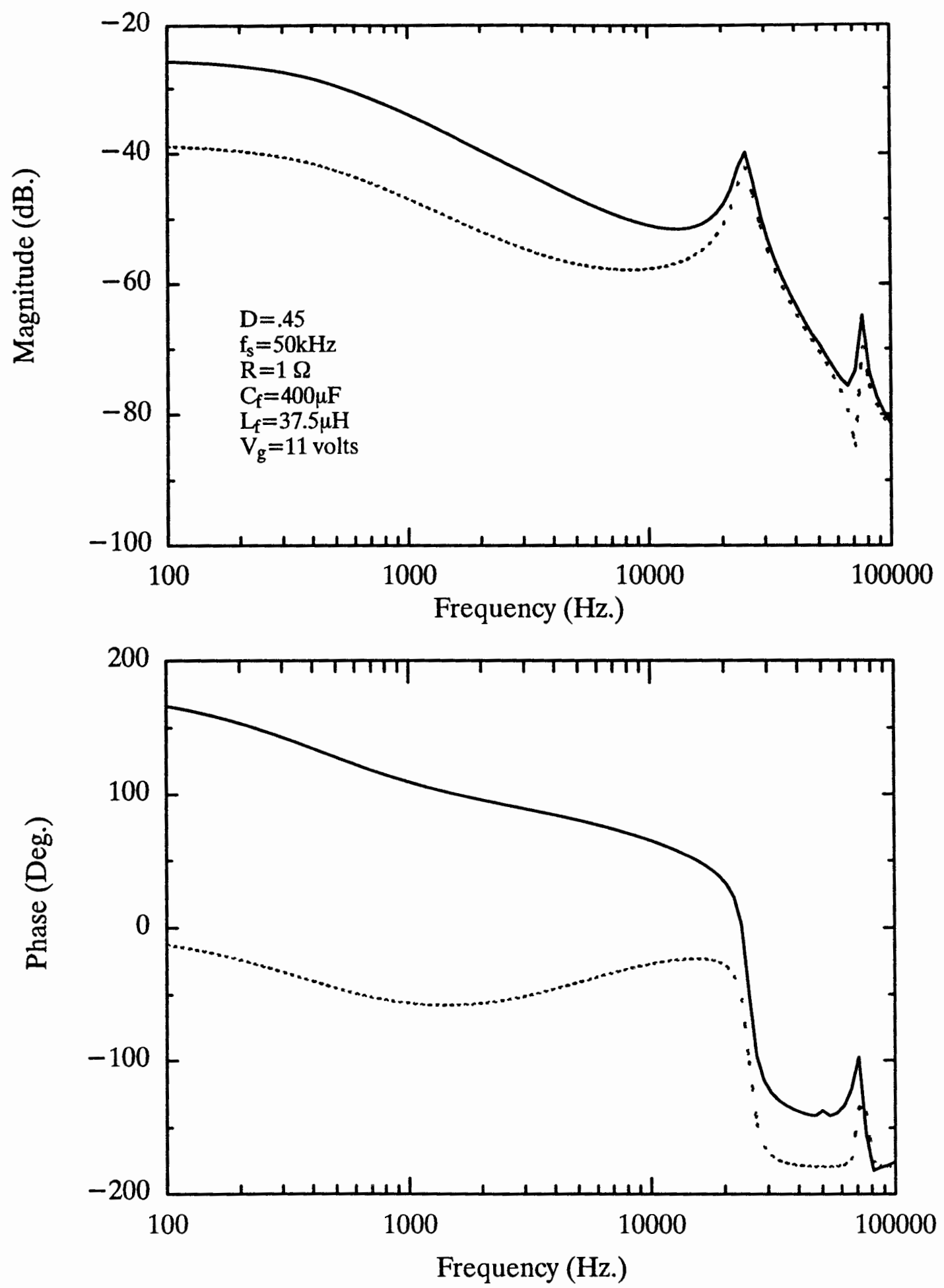

Figure 5-22 The input-to-output response of the PWM buck converter operating in CCM under current mode programming; exact (solid line) versus the second model (dashed line). 


\subsection{Buckboost Converter Analysis}

In this section, the hybrid switch model is used to predict the small signal frequency response of a buckboost converter. The model results are compared against the exact ones. The analysis done here are for the duty ratio programmed and current programmed buckboost converters operating in CCM.

\subsubsection{PWM Duty Ratio Programmed Buckboost Converter}

Figure 5 - 23 shows a basic PWM buckboost converter. For small signal analysis of the converter, the PWM switch has been replaced by the hybrid parameter switch model as shown in Figure $5-24$.

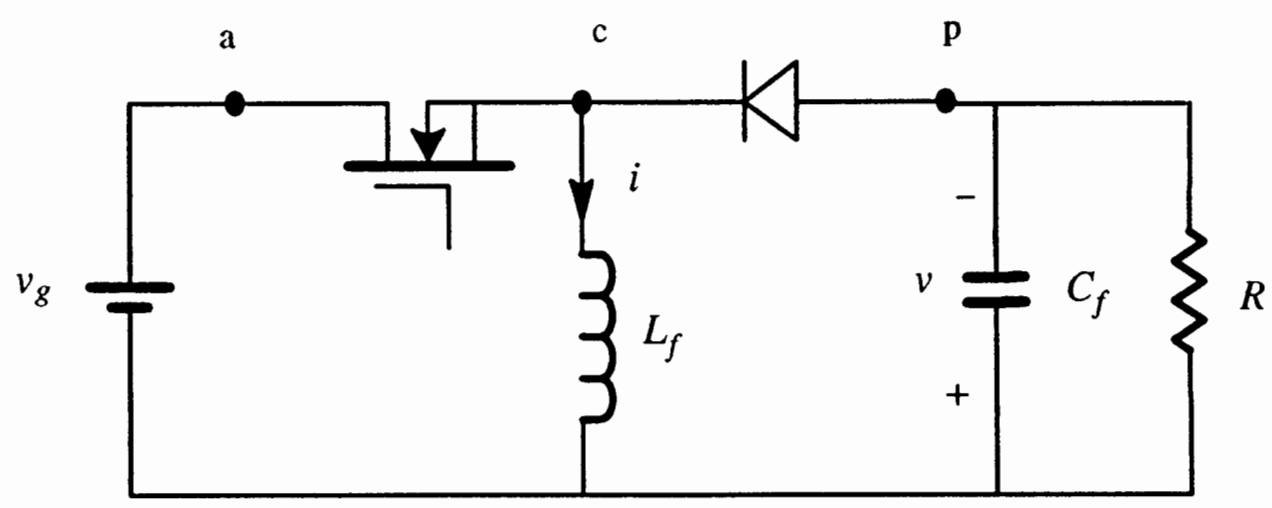

Figure 5 - 23 Circuit schematics for PWM buckboost converter. 


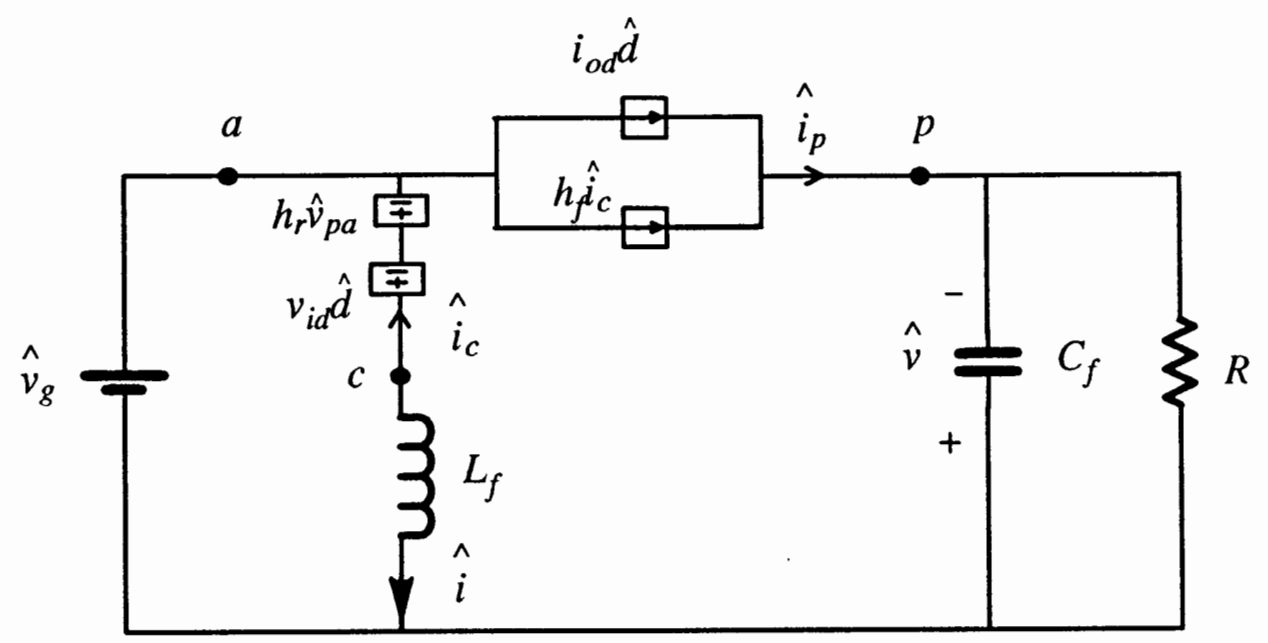

Figure 5-24 Equivalent small - signal hybrid parameter switch model for the PWM buckboost converter of Figure 5-23.

The dc operating point conditions are deduced from Figure 5-23. They are:

$$
\begin{aligned}
& I_{c}=-I \\
& V_{p a}=-\left(V_{g}+V\right)
\end{aligned}
$$

The small signal perturbation terms found from Figure 5 -24 are:

$$
\hat{i_{c}}=-\hat{i}
$$

and

$$
\hat{v}_{p a}=-\left(\hat{v}_{g}+\hat{v}\right)
$$

To obtain the small-signal control-to-output transfer function, all of the independent sources except $\hat{d}$ need to be deactivated. Therefore, in Figure $5-24, \hat{v}_{g}$ will be shorted and as a result this model reduces to the one shown in Figure $5-25$. 


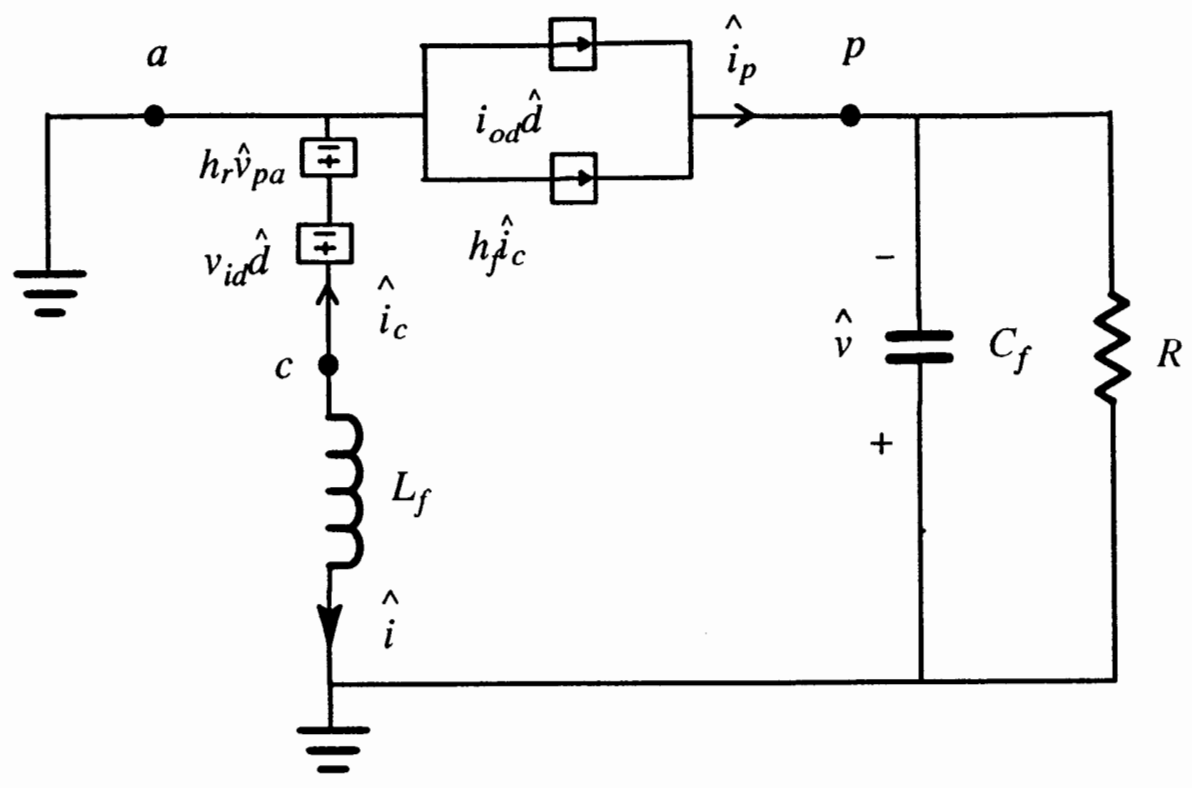

Figure 5-25 Equivalent small-signal control-to-output hybrid parameter switch model for PWM buckboost converter of Figure 5-23.

Hence, equation $(5-75)$ will reduce to:

$$
\hat{v}_{p a}=-\hat{v}
$$

Furthermore, using KVL one gets:

$$
-h_{r} \hat{v}_{p a}-v_{i d} \hat{d}+\frac{\hat{d i}}{d t} L_{f}=0
$$

and substituting equation (5 - 76) into equation $(5-77)$ and simplifying gives:

$\frac{\hat{d i}}{d t}=-\frac{h_{r}}{L_{f}} \hat{v}+\frac{v_{i d}}{L_{f}} \hat{d}$

Employing KCL will give:

$$
\hat{i}_{p}+\frac{d \hat{v}}{d t} C_{f}+\frac{\hat{v}}{R}=0
$$


It is also known that

$$
\hat{i_{p}}=i_{o d} \hat{d}+h_{f} \hat{i_{c}}
$$

Substituting equation $(5-80)$ into equation $(5-79)$ leads to :

$$
i_{o d} \hat{d}+h_{f} \hat{i_{c}}+\frac{\hat{d} \hat{v}}{d t} C_{f}+\frac{\hat{v}}{R}=0
$$

Substitution of equation $(5-74)$ into equation $(5-81)$ and rearranging gives :

$$
\frac{d \hat{v}}{d t}=-\frac{i_{o d}}{C_{f}} \hat{d}+\frac{h_{f}}{C_{f}} \hat{i}-\frac{1}{R C_{f}} \hat{v}
$$

State equations $(5-78)$ and $(5-82)$ are rewritten in the state equation matrix form :

$$
\frac{d}{d t}\left[\begin{array}{l}
\hat{i} \\
\hat{v}
\end{array}\right]=\left[\begin{array}{ll}
0 & -\frac{h_{r}}{L_{f}} \\
\frac{h_{f}}{C_{f}}-\frac{1}{R C_{f}}
\end{array}\right]\left[\begin{array}{l}
\hat{i} \\
\hat{v}
\end{array}\right]+\left[\begin{array}{r}
\frac{v_{i d}}{L_{f}} \\
-\frac{i_{o d}}{C_{f}}
\end{array}\right] \hat{d}
$$

The input to output equivalent small-signal model depicted in Figure 5-26 is obtained by deactivating the controller signal. The input - to-output transfer function of Figure 5-26 may also be found using state space analysis of the system. 


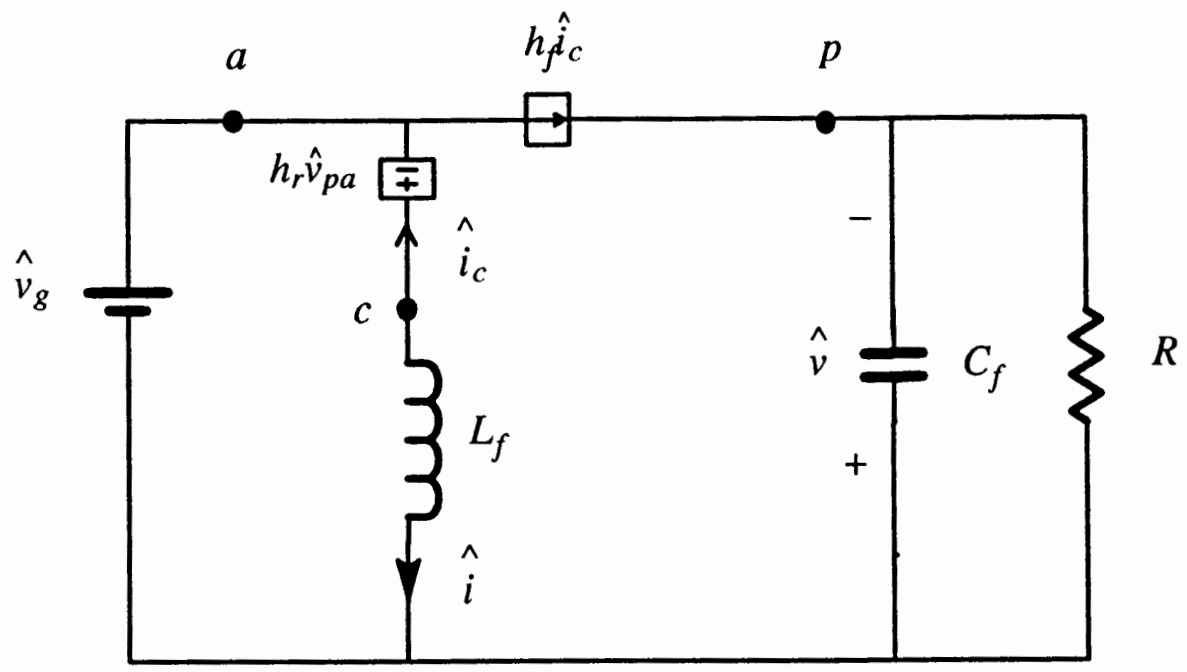

Figure 5 - 26 Equivalent input-to-ouput small-signal hybrid parameter switch model for the PWM buckboost converter of Figure $5-23$.

Applying KVL will give :

$-\hat{v}_{g}-h_{r} \hat{v}_{p a}+\frac{\hat{d i}}{d t} L_{f}=0$

Substituting equation $(5-75)$ into equation $(5-84)$ and rearranging will give:

$\frac{\hat{d i}}{d t}=-\frac{h_{r}}{L_{f}} \hat{v}+\frac{1-h_{r}}{L_{f}} \hat{v_{g}}$

Similarly, the second equation is formed using $\mathrm{KCL}$ :

$\hat{i_{p}}+\frac{d \hat{v}}{d t} C_{f}+\frac{\hat{v}}{R}=0$

Using

$\hat{i}_{p}=h_{i_{c}}$

The substitution of equation $(5-87)$ into equation $(5-86)$ gives :

$\hat{h}_{f_{c}}+\frac{d \hat{v}}{d t} C_{f}+\frac{\hat{v}}{R}=0$ 
Using equation $(5-74)$ to simplify equation $(5-88)$ will give:

$$
\frac{d \hat{v}}{d t}=\frac{d}{C_{f}} \hat{i}-\frac{1}{R C_{f}} \hat{v}
$$

and input-to-output matrices are :

$$
\frac{d}{d t}\left[\begin{array}{l}
\hat{i} \\
\hat{v}
\end{array}\right]=\left[\begin{array}{cc}
0 & -\frac{h_{r}}{L_{f}} \\
\frac{h_{f}}{C_{f}}-\frac{1}{R C_{f}}
\end{array}\right]\left[\begin{array}{l}
\hat{i} \\
\hat{v}
\end{array}\right]+\left[\begin{array}{c}
\frac{1-h_{r}}{L_{f}} \\
0
\end{array}\right] \hat{v_{g}}
$$

The accuracy of the above results is examined by comparing its frequency response against the exact plots, shown in Figure $5-27$ and Figure $5-28$. The percentage ripples are found to be:

$$
\begin{aligned}
& \frac{\Delta v / 2}{V} \times 100 \approx 1.3 \% \\
& \frac{\Delta i / 2}{I} \times 100 \approx 9.5 \%
\end{aligned}
$$

From Figure $5-27$ it can be observed that the control to output response is accurate even beyond half the switching frequency, $f_{s} / 2$. It is evident from Figure $5-28$ that the input to output magnitude response is very accurate up to $f_{s} / 2$. The phase associated with the input to output response of the model is exact up to the switching frequency, $f_{s}$. 

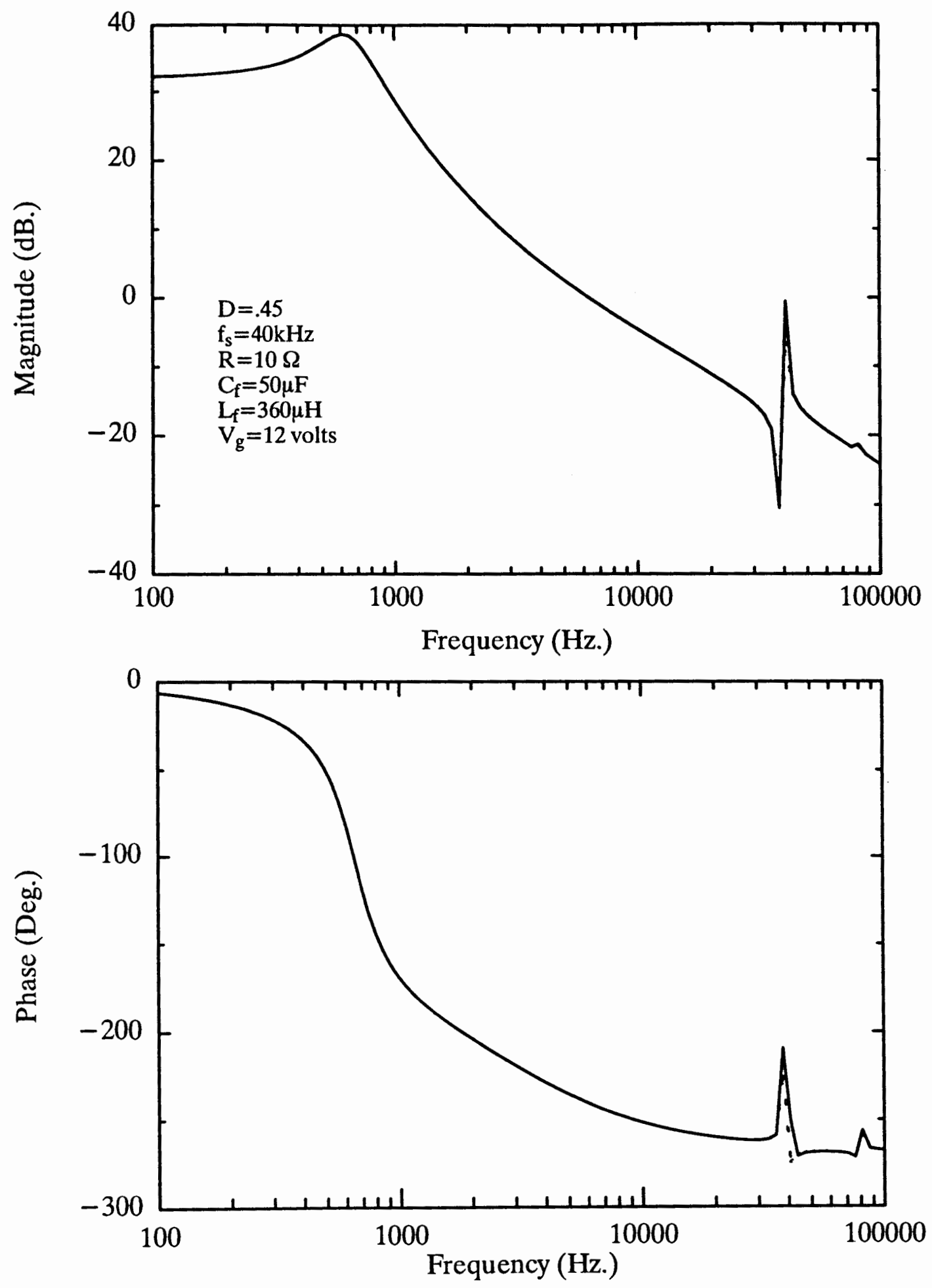

Figure 5-27 The control-to-output response of the PWM buck-boost converter operating in CCM; exact (solid line) versus the second model (dashed line). 

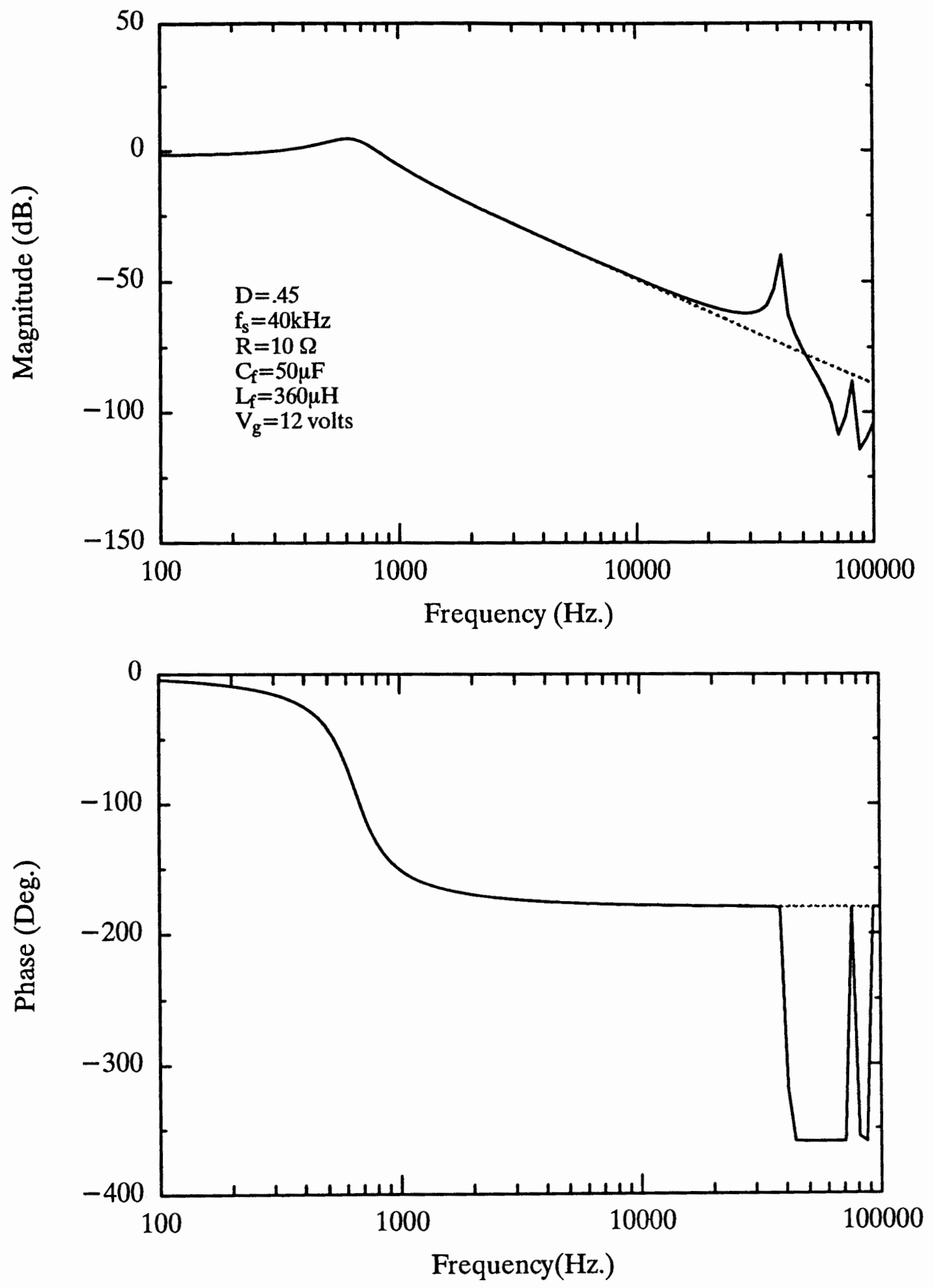

Figure 5-28 The input-to-output response of the PWM buck-boost converter operating in CCM; exact (solid line) versus the second model (dashed line). 


\subsubsection{PWM Current Programmed Buckboost Converter}

Figure 5 - 29 represents a basic buckboost converter operating in current mode programming. Replacing the switch by its equivalent current mode hybrid model will result in Figure $5-30$.

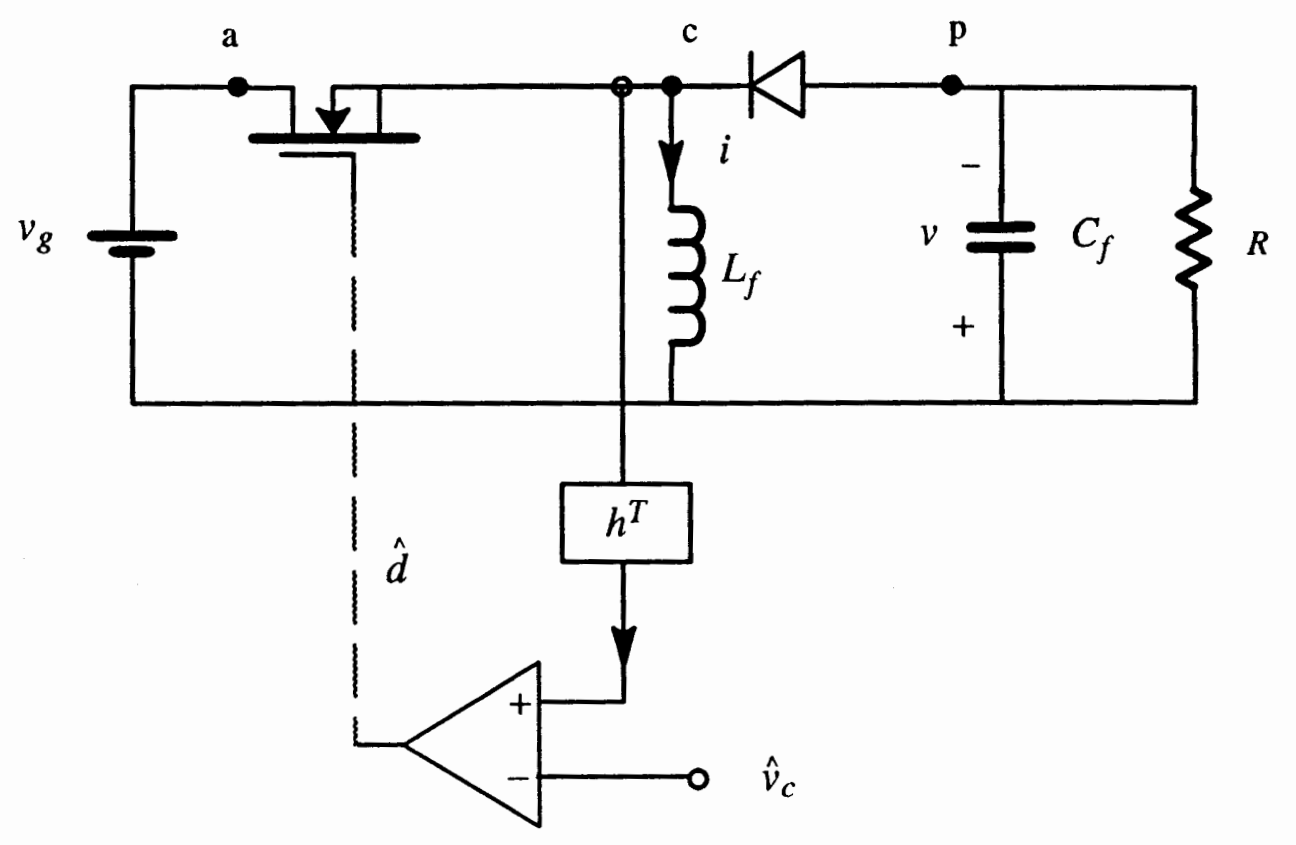

Figure 5 - 29 Circuit schematics for PWM buckboost converter operating in current programming mode.

From Figure 5-29, the slope of the rising edge of the inductor current is found to be

$$
m_{1}=\frac{V_{g}}{L_{f}}
$$

In this circuit, no compensation ramp has been used, therefore, substituting equation $(5-91)$ into equation $(5-61)$ will give:

$$
\mu=\frac{L_{f}}{h^{T} V_{g}}
$$

Next $\Gamma$ can be found, but before that $\xi$ has to be identified. 


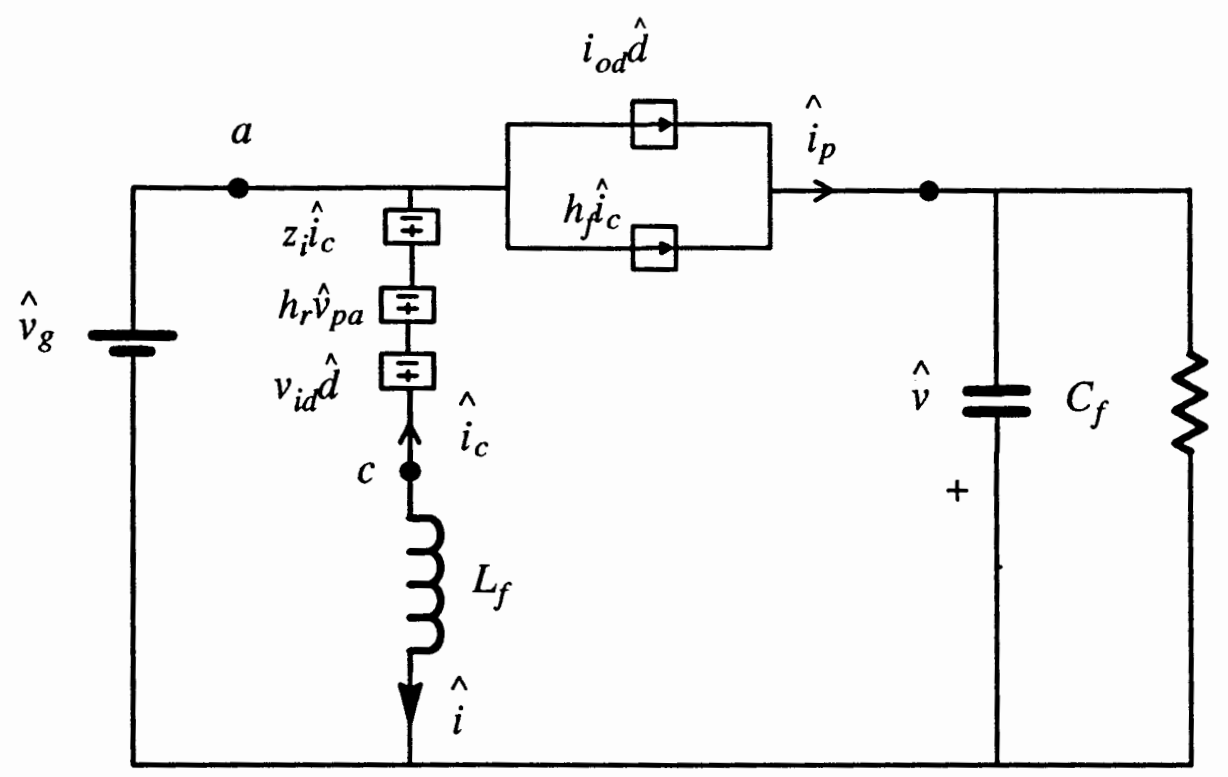

Figure 5-30 Equivalent small-signal hybrid parameter switch model for current mode analysis of the buckboost converter of Figure $5-23$.

From $(3-48)$ one finds:

$$
\xi=\frac{V_{g}}{L_{f}}+\frac{V}{L_{f}}
$$

The steady state output capacitor voltage is actually related to the input voltage by the dc conversion ratio. For the buckboost converter used in this example,

$$
V=\frac{V_{g} D}{D^{\prime}}
$$

Substituting equation $(5-94)$ in equation $(5-93)$ will result in:

$$
\xi=\frac{V_{g}}{L_{f}}\left(1+\frac{D}{D^{\prime}}\right)
$$

and substituting equations $(5-92)$ and $(5-95)$ into equation $(5-62)$ gives:

$$
\Gamma=1-\frac{V_{g}}{L_{f}}\left(1+\frac{D}{D^{\prime}}\right) h^{T} \frac{L_{f}}{h^{T} V_{g}}
$$


which simplifies to

$$
\Gamma=-\frac{D}{D^{\prime}}
$$

In the buckboost converter of Figure 5 - 30, the common terminal current is flowing towards the opposite side of the inductor current as shown in equation (5-74). This mean that the sign of $h^{T}$ must be changed so it would be consistent with the direction of the inductor current. Therefore, equation $(5-70)$ holds true for the buckboost converter as well.

Figure 5 - 30 could be used to find the small-signal state equations of the system. The current programmed model is essentially the same as the duty ratio model with the addition of the $z_{i}$ parameter. Therefore, the only change will be in the A matrix which is given below:

$$
A=\left[\begin{array}{cc}
-\frac{z_{i}}{L_{f}} & -\frac{h_{r}}{L_{f}} \\
\frac{h_{f}}{C_{f}} & -\frac{1}{R C_{f}}
\end{array}\right]
$$

From Figure 5 - 31, one can see that the control-to-output response is very accurate; therefore, $v_{i d}$ and $i_{o d}$ are exact and give good results. Figure 5-32 shows that the input to output parameters do not produce satisfactory results, especially in the phase plot. The capacitor voltage and inductor current ripples are found to be:

$$
\begin{aligned}
& \frac{\Delta v / 2}{V} \times 100 \approx 10 \% \\
& \frac{\Delta i / 2}{I} \times 100 \approx 11 \%
\end{aligned}
$$

The input-to-output responses of both the buck and buckboost converters using the second model were inaccurate. In the next chapter, a new approach is presented which leads to accurate results for the input-to-output responses of different systems. 

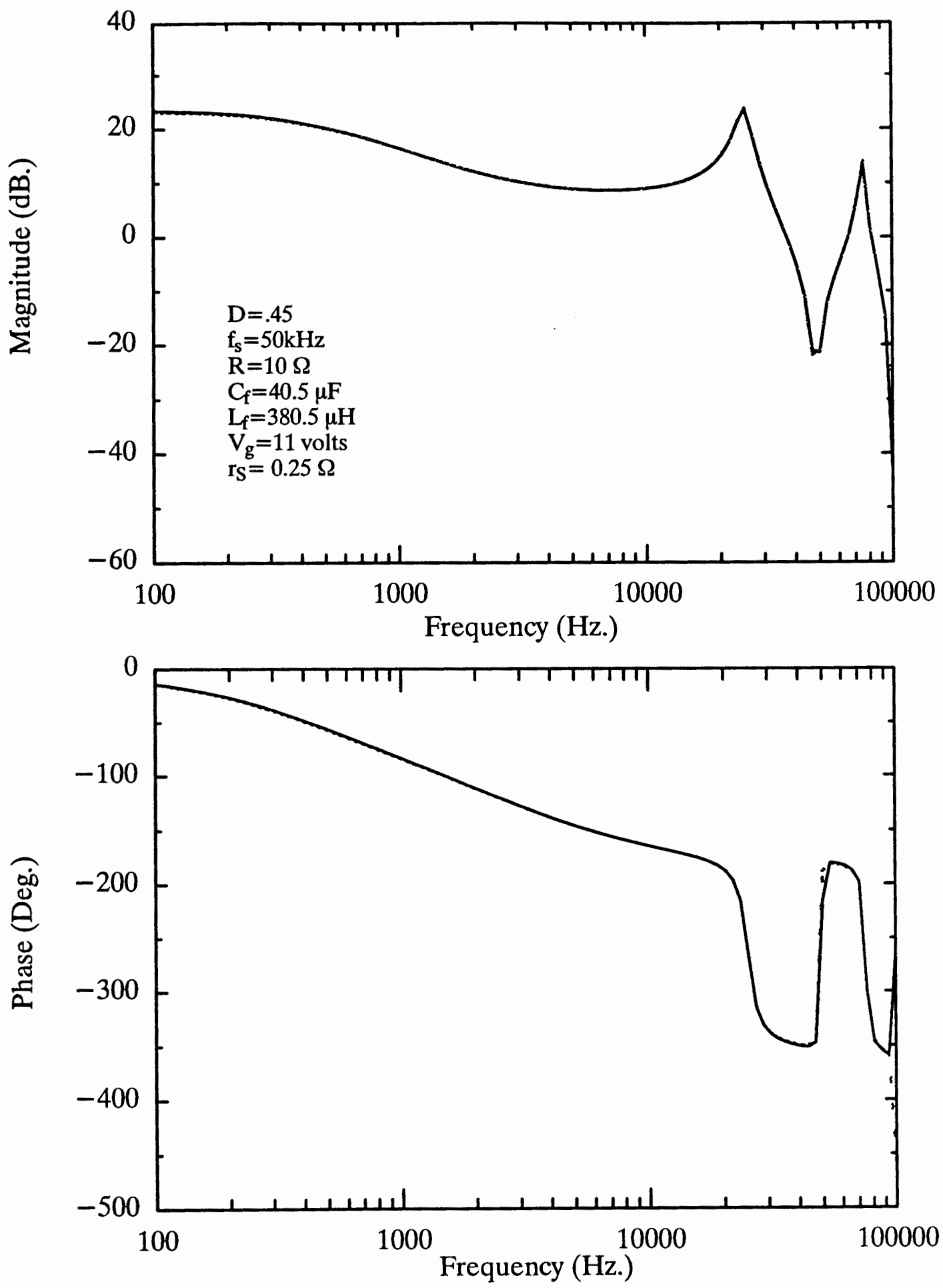

Figure 5-31 The control-to-output response of the PWM buckboost converter operating in CCM under current mode programming; exact (solid line) versus the second model (dashed line). 

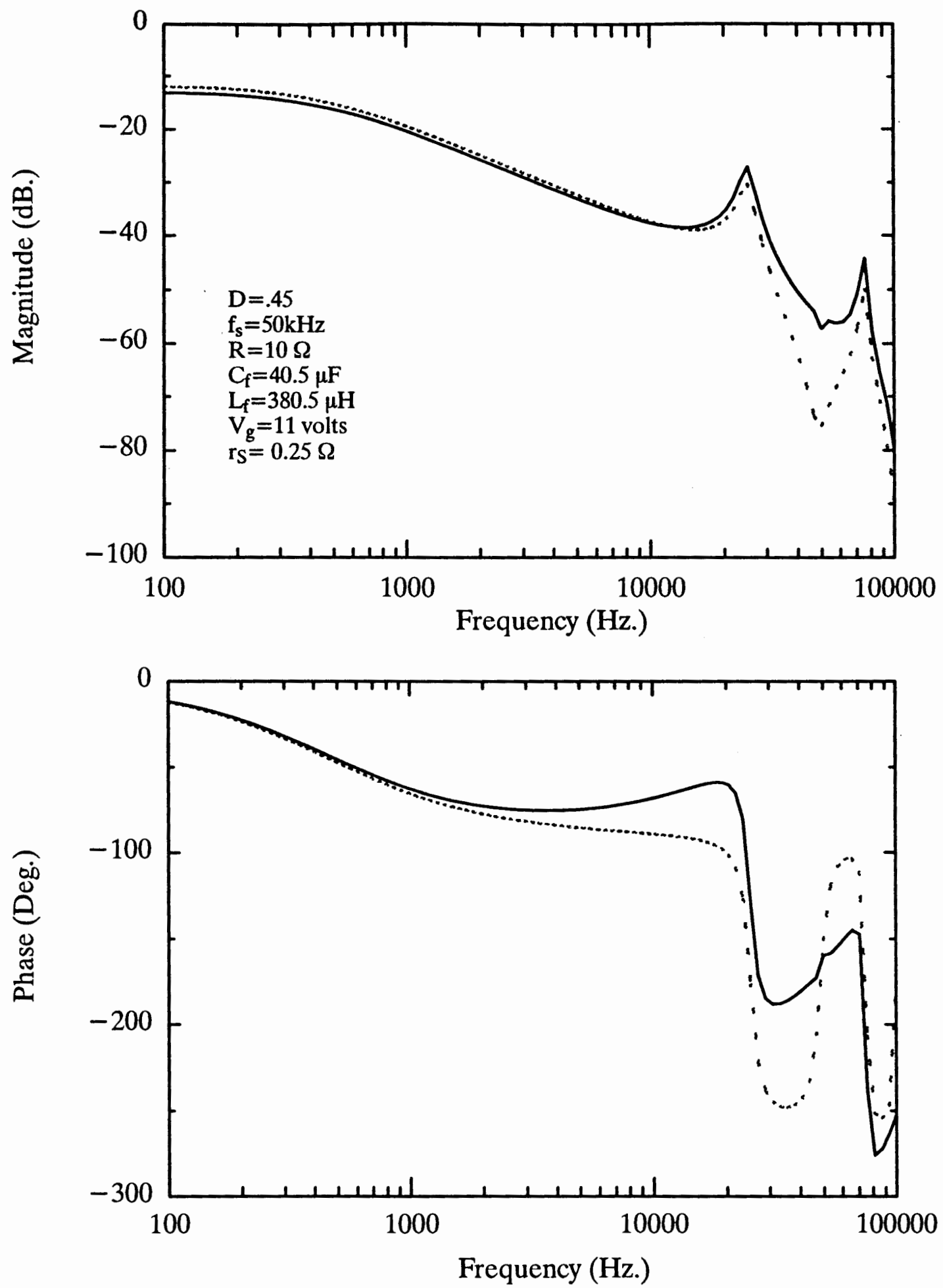

Figure 5- 32 The input - to-output response of the PWM buckboost converter operating in CCM under current mode programming; exact (solid line) versus the second model (dashed line). 


\section{CHAPTER 6 \\ THIRD MODEL: NEW METHOD FOR OBTAINING INPUT PARAMETERS}

\subsection{General Analysis of the Model}

The model found in the previous chapter failed to accurately predict the input-tooutput response of current mode programmed converters. Therefore, a new approach is undertaken which produces exact but more complicated expressions.

Since input to output parameters, $h_{m} z_{i}, h_{f}$, and $y_{o}$, obtained in the last modeling approach gave inaccurate results, an attempt is made to alter the procedure for finding these parameters.

The controller terms in equations $(2-41)$ and $(2-42)$ do not contribute to the input to output parameters. Therefore, when finding the input terms, the equations $(2-41)$ and $(2-42)$ can be found by deactivating the effect of the controller input; i.e.

$$
\begin{aligned}
& \left.\hat{v}_{c a}\right|_{\hat{d}=0}=h_{r} \hat{v}_{p a}+z_{i} \hat{i}_{c} \\
& \left.\hat{i}_{p}\right|_{\hat{d}=0}=h_{f} \hat{i}_{c}+y_{o} \hat{v}_{p a}
\end{aligned}
$$

In equation $(6-1)$, there are two unknown parameters. If one of the parameters is found by nulling, then there will be one equation and one unknown. So it is possible to solve for the second parameter. The question is which parameter should be subjected 
to nulling in order to produce accurate results both for the full order and reduced order analysis?

The fact that the number of states is reduced to a single inductor state imposed more restriction on the proposed current programmed model. That was the reason for which the previous model did not produce accurate results. To clarify this, let us look at the $h_{r}$ parameter.

$$
h_{r}=\frac{\hat{v}_{c a}}{\hat{v}_{p a}} \mid \begin{aligned}
& \hat{d}=0 \\
& \hat{i_{c}}=0
\end{aligned}
$$

The exact input - to-output transfer function given in equation $(3-52)$ is used to obtain the $h_{r}$ parameter. First the inductor current state $\hat{i}_{l}$ must be nulled. Noting that $h^{T}$ is the term which isolates the inductor current state, $i_{l}=h^{T} x$. Therefore, the terms containing $h^{T}$ should also be nulled. One of the places in equation $(3-52)$ the effect of the sensed current appears in is

$$
\zeta h^{T} \mu \beta_{1} \beta_{2}
$$

When the number of states was reduced, the contribution of the sensed current towards the second state disappeared in expression $(6-3)$. To clarify this, consider the full order model with

$$
B=\beta_{1} \beta_{2}
$$

where $B$ is a $2 \times 2$ matrix given as:

$$
B=\left[\begin{array}{ll}
b_{11} & b_{12} \\
b_{21} & b_{22}
\end{array}\right]
$$

$h^{T}$ is a $1 \times 2$ vector containing only $R_{i}$ which is a current to voltage transformation gain.

$$
h^{T}=\left[\begin{array}{ll}
R_{i} & 0
\end{array}\right]
$$


$\zeta$ and $\mu$ are scalars, therefore the expression $(6-3)$ can be expanded as follows:

$$
\zeta \mu\left[\begin{array}{ll}
R_{i} b_{11} & R_{i} b_{12}
\end{array}\right]
$$

The reduced order model does not contain the following term:

$$
\zeta \mu R_{i} b_{12}
$$

and if $\hat{i}_{c}$ is nulled in the reduced model, equation $(6-8)$ will be reduced to zero. Therefore, equation $(6-3)$ also goes to zero.

In general, when $\hat{i}_{c}$ is nulled, equation $(3-52)$ will reduce to the following:

$$
\frac{\hat{y}}{\hat{u_{i}}}(j \omega)=\frac{1}{T_{s}}\left\{E_{2}\left(T_{3}-T_{2}\right)\right\}
$$

Hence using equation $(6-9)$, the $h_{r}$ parameter reduces to:

$$
h_{r}=\frac{\hat{y}}{\hat{u}_{i}}(j \omega)=\frac{1}{T_{s}}\left\{D^{\prime} T_{s}\right\}=D^{\prime}
$$

Equation $(6-9)$ was used in obtaining two of the input - to-output parameters of the second model. Furthermore, the proposed model did not give accurate results when tested on different converters. Therefore, a new method is tried. Now, the main concern in the reduced order analysis is to avoid nulling the inductor current state. Hence, the other parameter in equation $(6-1)$, namely $z_{i}$, will be subjected to nullification.

The $z_{i}$ parameter is given in expression $(2-47)$ which is also repeated here for reference:

$$
z_{i}=\frac{\hat{v}_{c a}}{\hat{i_{c}}} \mid \begin{aligned}
& \hat{d}=0 \\
& \hat{v}_{p a}=0
\end{aligned}
$$


Since both the numerator and the denominator of the $z_{i}$ parameter are outputs, expression $(2-47)$ can be written in the following form:

$$
z_{i}=\frac{\hat{v}_{c a} / \hat{v}_{g}}{\hat{i}_{c} / \hat{v}_{g}} \quad \begin{gathered}
\hat{d}=0 \\
\hat{v}_{p a}=0
\end{gathered}
$$

After finding the $z_{i}$ parameter by deactivating the effect of $\hat{v}_{p a}$, the $h_{r}$ parameter can be found using the following method:

Dividing equation $(6-1)$ by $\hat{v}_{p a}$ will give:

$$
\left.\frac{\hat{v}_{c a}}{\hat{v}_{p a}}\right|_{\hat{d}=0}=h_{r}+\left.z_{i} \frac{\hat{i}_{c}}{\hat{v}_{p a}}\right|_{\hat{d}=0}
$$

Rearranging equation $(6-12)$ results in:

$$
h_{r}=\left.\frac{\hat{v}_{c a}}{\hat{v}_{p a}}\right|_{\hat{d}=0}-\left.z_{i} \frac{\hat{i}_{c}}{\hat{v}_{p a}}\right|_{\hat{d}=0}
$$

The same procedure is applied to equation $(6-2)$. The $h_{f}$ parameter is obtained first by nulling $\hat{v}_{p a}$. Then dividing equation $(6-2)$ by $\hat{v}_{p a}$ and rearranging the terms gives:

$$
y_{o}=\left.\frac{\hat{i}_{p}}{\hat{v}_{p a}}\right|_{\hat{d}=0}-\left.h_{f} \frac{\hat{i}_{c}}{\hat{v}_{p a}}\right|_{\hat{d}=0}
$$

The controller parameters are then found by substituting equation $(6-13)$ into equation $(5-1)$ and substituting equation $(6-14)$ into equation $(5-2)$. Therefore, the new controller parameter $v_{i d}$ is:

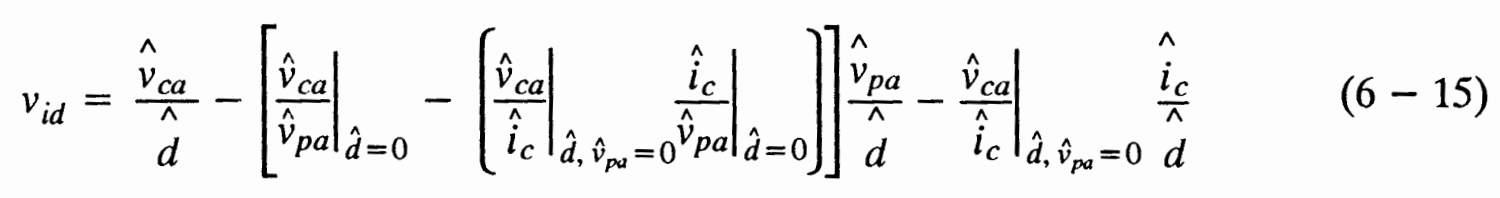

and $i_{o d}$ is given by 


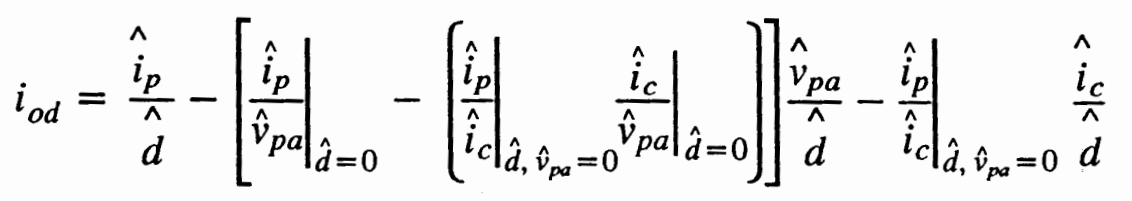

Equations $(6-15)$ and $(6-16)$ have been derived under one important constraint. Namely, the input to output parameters are obtained by using a different condition than the control to output parameters. Under this assumption, equations $(6-15)$ and $(6-16)$ will be valid.

Equations $(6-13)$ and $(6-14)$ can be written in a simpler manner.

$$
\begin{aligned}
& h_{r}=h_{r}{ }^{\prime}-z_{i} y_{r} \\
& y_{o}=y_{o}{ }^{\prime}-h_{f} y_{r}
\end{aligned}
$$

where

$$
\begin{aligned}
& h_{r}^{\prime}=\left.\frac{\hat{v}_{c a}}{\hat{v}_{p a}}\right|_{\hat{d}=0} \\
& y_{o}^{\prime}=\left.\frac{\hat{i_{p}}}{\hat{v}_{p a}}\right|_{\hat{d}=0} \\
& y_{r}=\left.\frac{\hat{i_{c}}}{\hat{v}_{p a}}\right|_{\hat{d}=0}
\end{aligned}
$$

The controller equations $(6-15)$ and $(6-16)$ can be given in the form of equations $(5-3)$ and $(5-4)$ which are:

$$
\begin{aligned}
& v_{i d}=v_{i d}{ }^{\prime}-h_{r} \cdot v_{o d}-z_{i} \cdot i_{i d} \\
& i_{o d}=i_{o d}{ }^{\prime}-y_{o} \cdot v_{o d}-h_{f} i_{i d}
\end{aligned}
$$

The next step in the development of the new model is to reduce the order of the system, so expressions will be easier to derive. The same assumption made in the last modeling approach are used here as well. It is assumed that the capacitor has a negligible voltage ripple, therefore, it is replaced with a constant voltage source. 


\subsubsection{PWM Duty Ratio Programmed Model}

First, the full order PWM duty ratio boost model is examined. It is shown in Figure $6-1$, and Figure $6-2$ that the control to output and input to output frequency responses of this model perfectly match the exact results. Next, the reduced order model will be examined. 

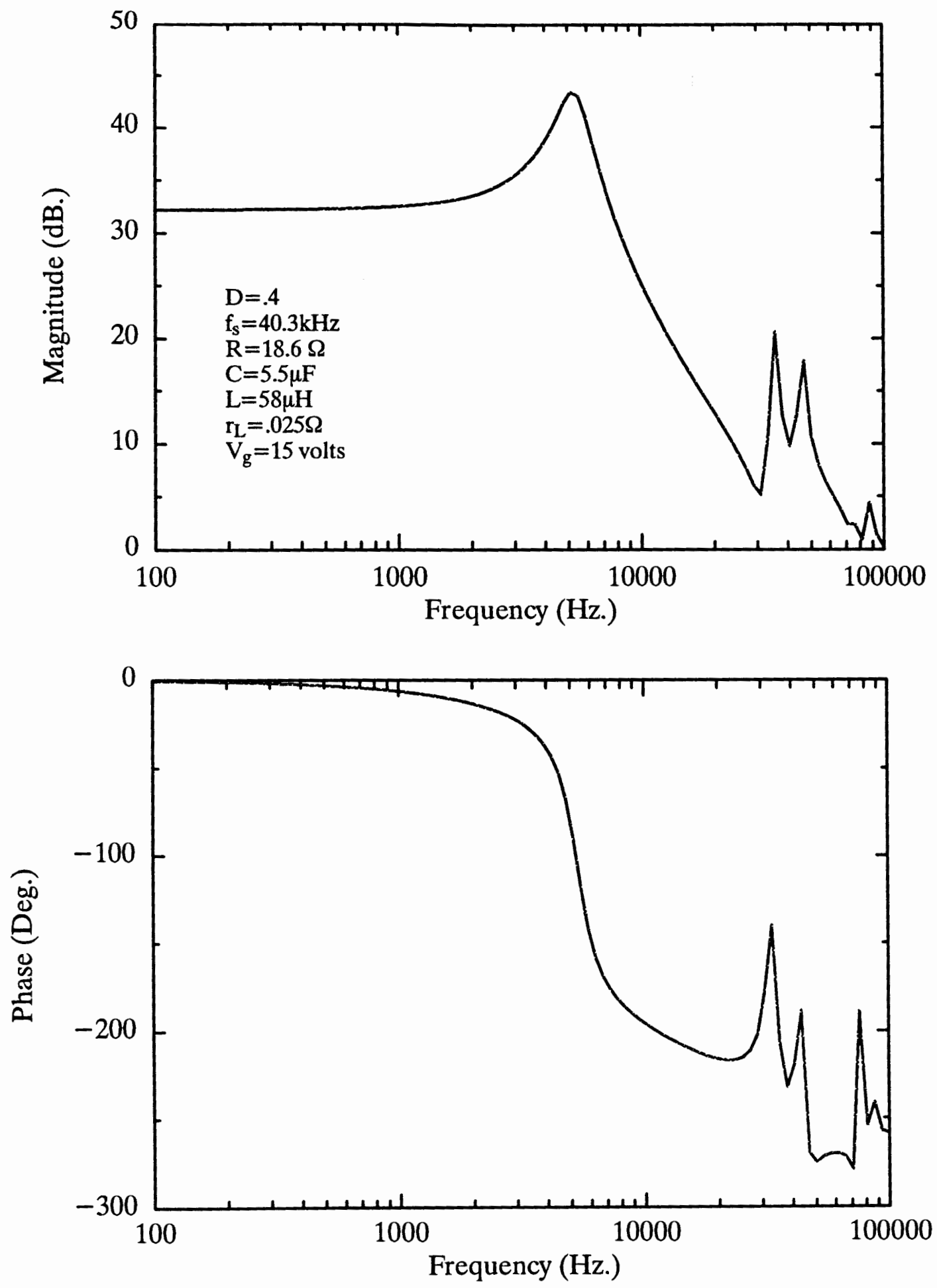

Figure 6-1 The control-to-output response of the PWM boost converter operating in CCM; exact (solid line) versus the third model (dashed line). 

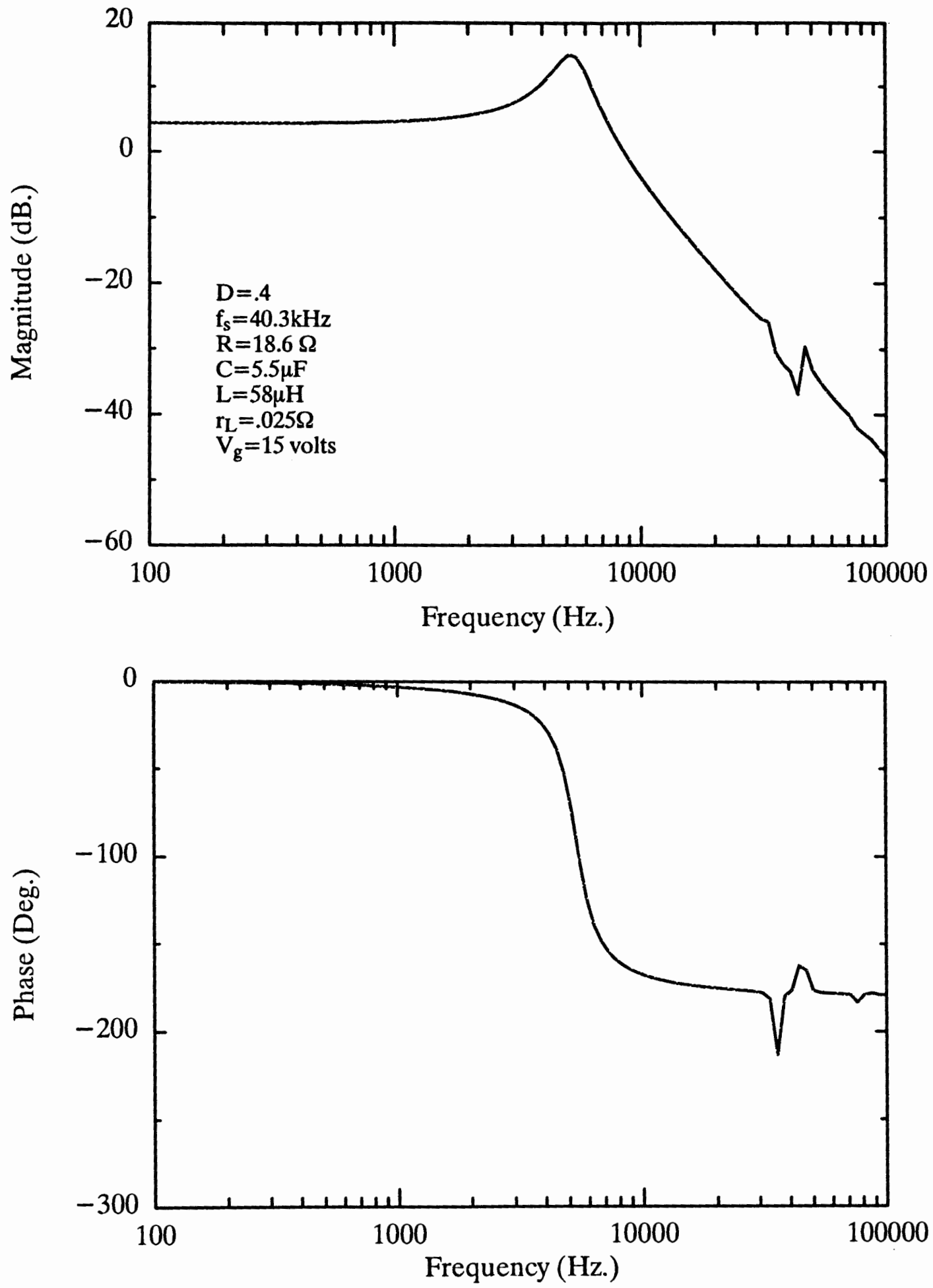

Figure $6-2$ The input-to-output response of the PWM boost converter operating in CCM; exact (solid line) versus the third model (dashed line). 
The control to output frequency response of the reduced PWM duty ratio boost model is plotted in Figure $6-4$. The component values are chosen to produce high ripple. The capacitor voltage ripple is around $4.85 \%$ of the DC voltage level. The inductor current ripple is chosen to be around $37.2 \%$ of the DC inductor current level. The low frequency magnitude of the model is shifted below the exact results by less than $1 \mathrm{~dB}$. At high frequencies, it is exact up to half of the switching frequency. The control to output phase plot is very accurate up to $f_{s} / 2$. The input-to-output frequency response of the new reduced model is plotted in Figure 6 - 5. As it can be observed, the magnitude and phase plots are exact everywhere except at the switching frequency.

In equation $(6-11)$, the line input, $\hat{v}_{g}$, to output transfer functions are used. This means only the $\hat{v}_{g}$ column of the input and the transmission matrices will be used for this analysis.

Next, the analytical solution for the parameters are found using Maple V. $v_{i d}{ }^{\prime}, z_{i}$, $i_{o d}{ }^{\prime}$, and $h_{f}$ parameters are obtained using the same technique as in the previous approach. Hence, they will not be repeated here. The new expressions are:

$$
\begin{aligned}
& h_{r}{ }^{\prime}=D^{\prime} \\
& y_{o}{ }^{\prime}=-\frac{\left(1-e^{\left.-j \omega D^{\prime} T_{s}\right)\left(1-e^{-j \omega D T_{s}}\right)}\right.}{\omega^{2} T_{s} L\left(1-e^{-j \omega T_{s}}\right)}+j \frac{D^{\prime}}{\omega L} \\
& y_{r}=j \frac{D^{\prime}}{\omega L}
\end{aligned}
$$

Using equation $(6-17)$ one gets:

$$
h_{r}=D^{\prime}
$$

and similarly the $y_{o}$ parameter is found by using equation $(6-18)$

$$
y_{o}=-\frac{\left(1-e^{-j \omega D^{\prime} T_{s}}\right)\left(1-e^{-j \omega D T_{s}}\right)}{\omega^{2} T_{s} L\left(1-e^{-j \omega T_{s}}\right)}+j \frac{D D^{\prime}}{\omega L}
$$


The new controller parameters, $v_{i d}$ and $i_{o d}$, are the same as those given in the previous modeling approach.

Since $z_{i}$ is zero, the new duty ratio hybrid parameter switch model reduces to the one shown in Figure $6-3$.

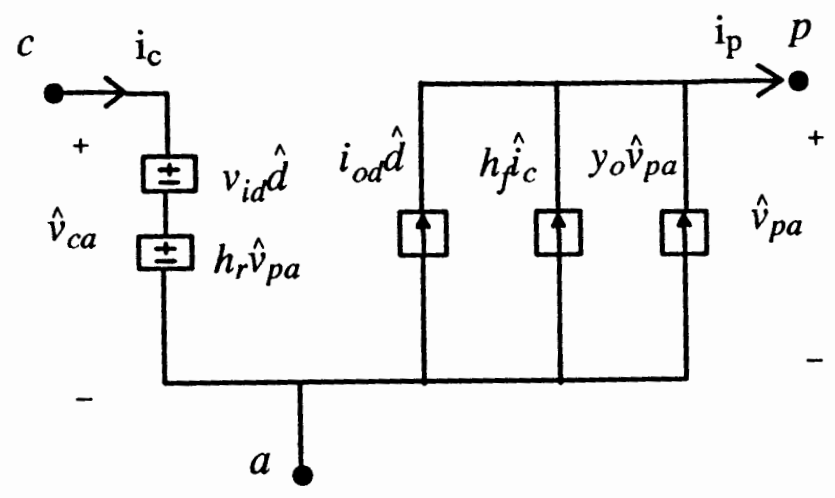

Figure 6-3 The hybrid parameter switch model for PWM duty ratio programming. 

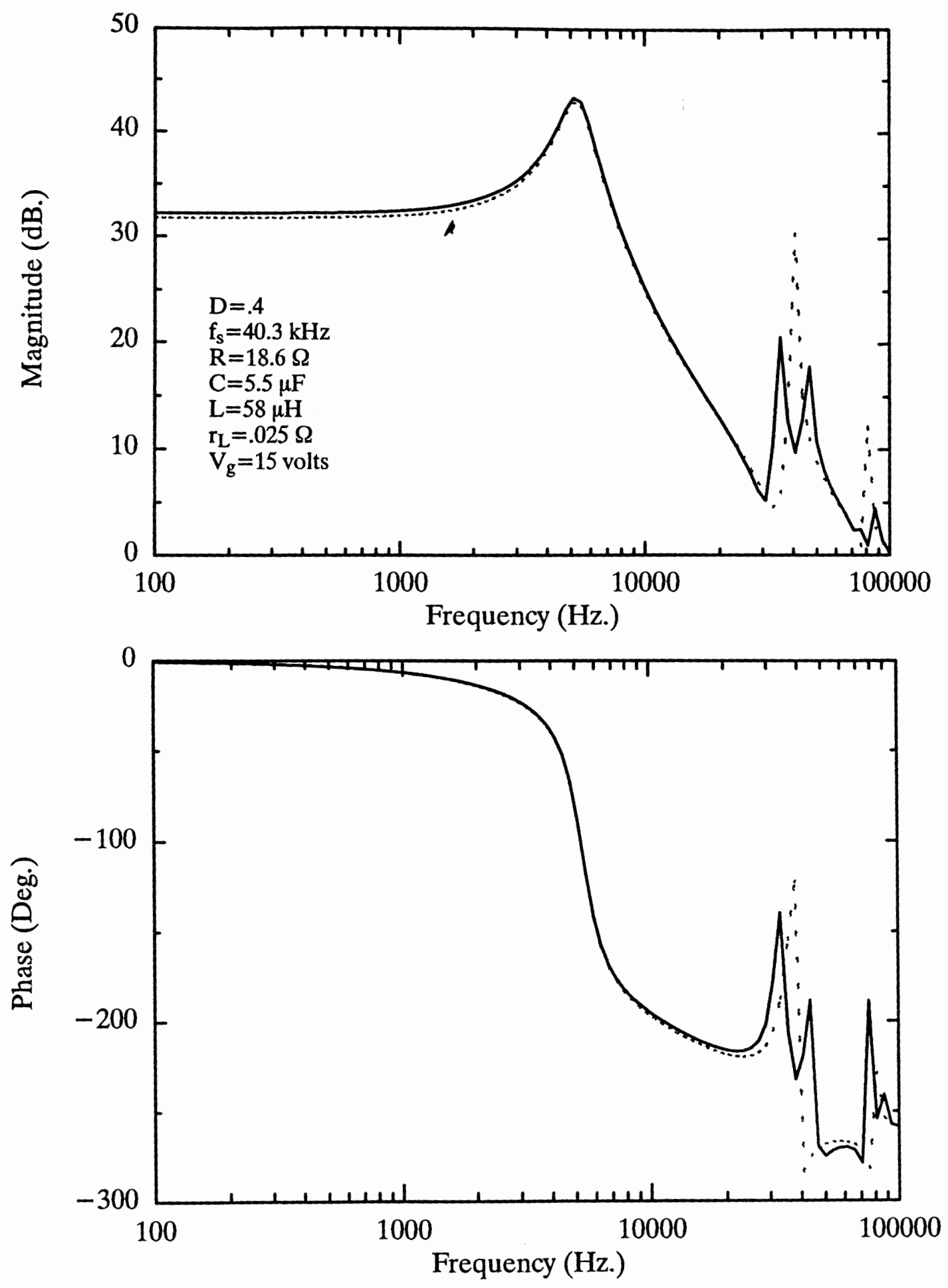

Figure 6-4 The control-to-output response of the PWM boost converter operating in CCM; exact (solid line) versus the reduced order third model (dashed line). 

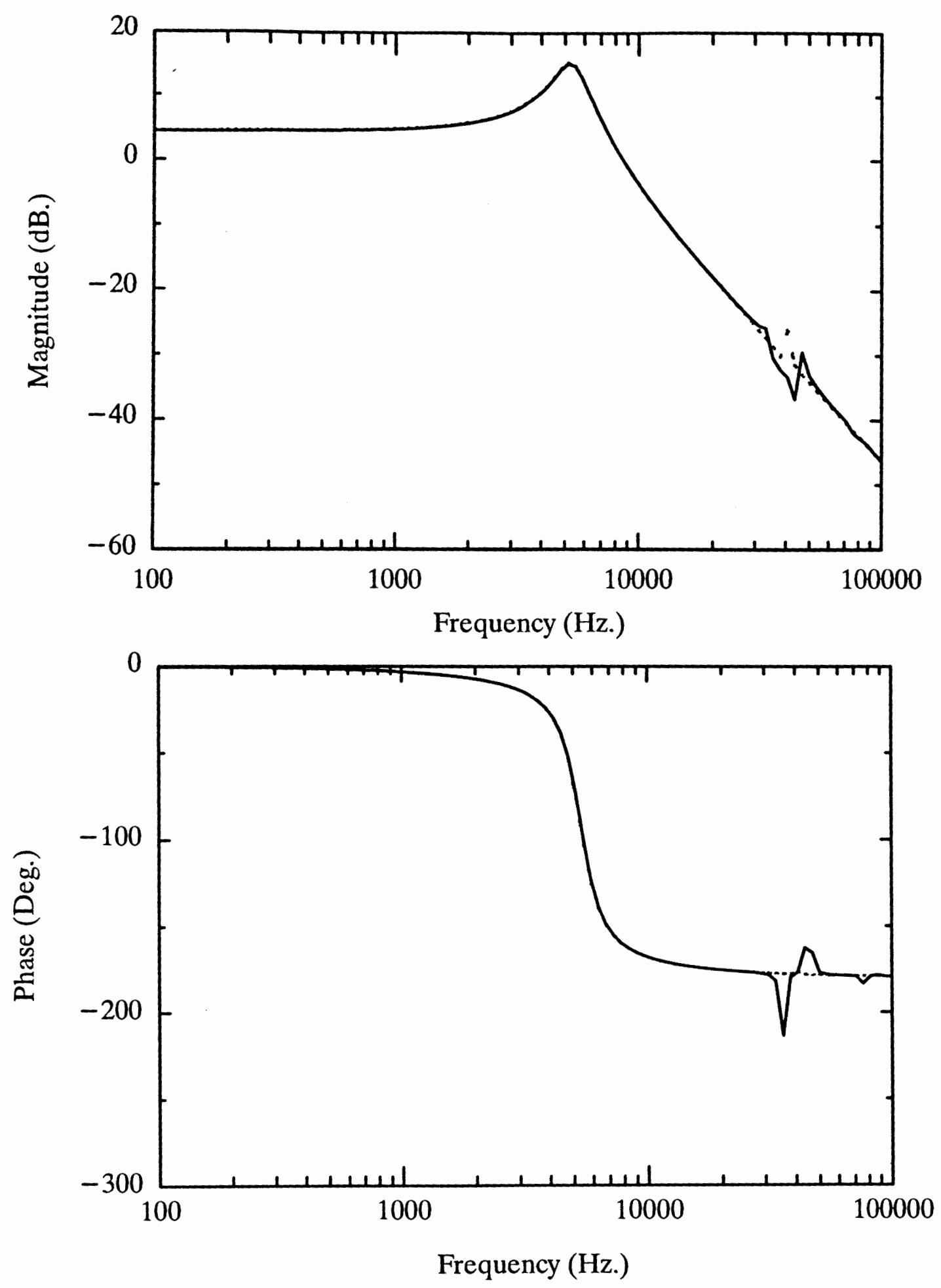

Figure 6 - 5 The input-to-output response of the PWM boost converter operating in CCM; exact (solid line) versus the reduced third model (dashed line). 


\subsubsection{PWM Current Programmed Model}

In section 6.1, a comprehensive hybrid parameter switch model was proposed. In this section the model will be examined for current programming case. The controlto-output magnitude and phase responses of the new model are shown in Figure $6-6$. The exact results are plotted on the same graph. The agreement is excellent. The input-to-output responses of the new model match that of the exact results as shown in Figure $6-7$.

The next step is to reduce the number of states in order to obtain simplified expressions. This is done by replacing the capacitor by a constant voltage source. The control to output and input to output transfer functions of the reduced model are plotted next.

The control to output transfer function of the new (third) reduced model is shown by the dashed line in Figure $6-8$. The theoretical prediction of the exact small signal control to output transfer function given by equation $(3-51)$ is shown by the solid line. A comparison of the plots shows an excellent agreement up to half of the switching frequency.

The input to output response is shown in Figure 6 - 9. The accuracy of the third model is shown to be very good. The magnitude plot follows the exact plot extremely well up to the switching frequency. The phase plot is also very accurate up to at least half the switching frequency. 

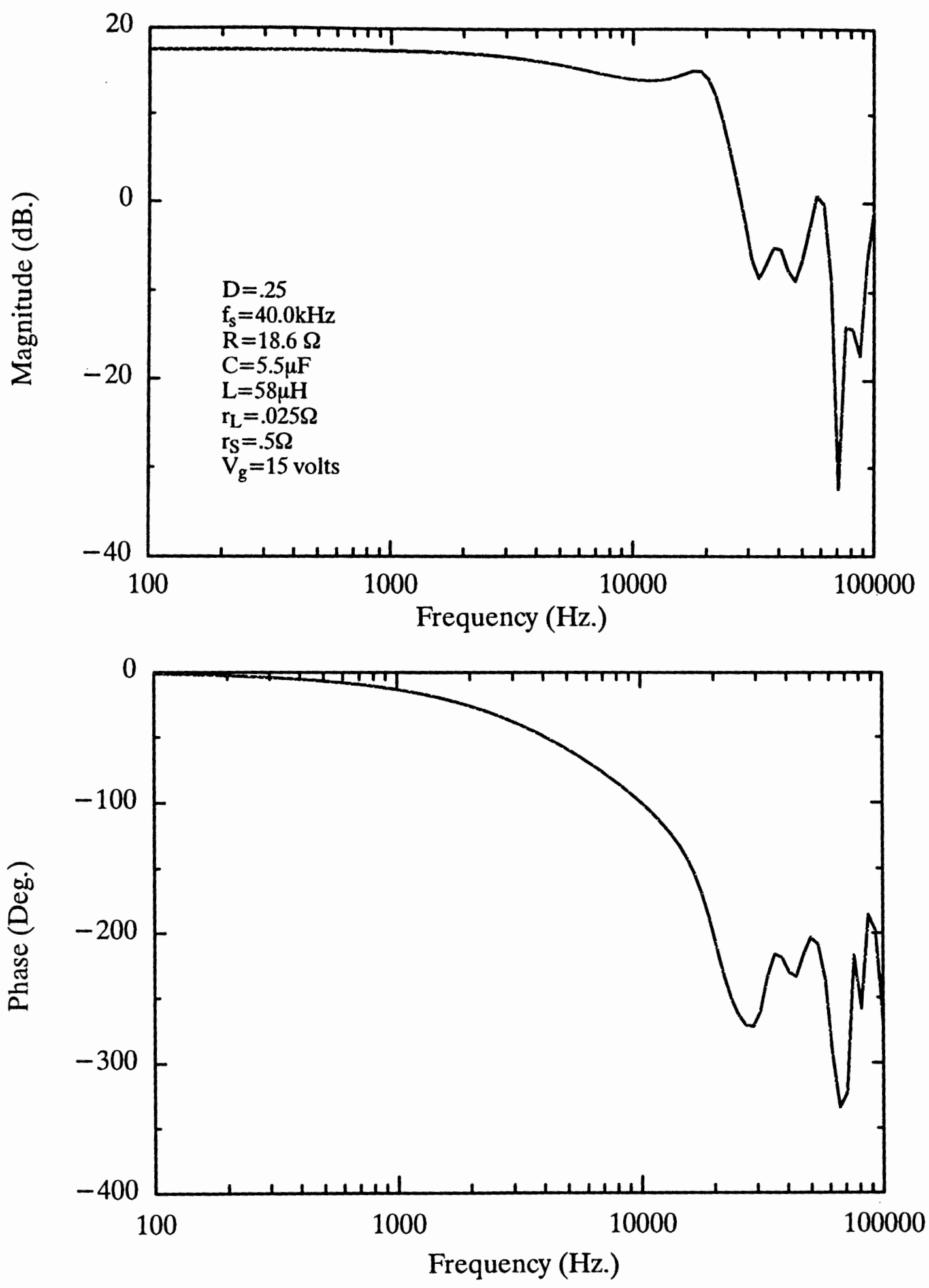

Figure 6- 6 The control-to-output response of the PWM boost converter operating in CCM under current mode programming; exact (solid line) versus the third model (dashed line). 

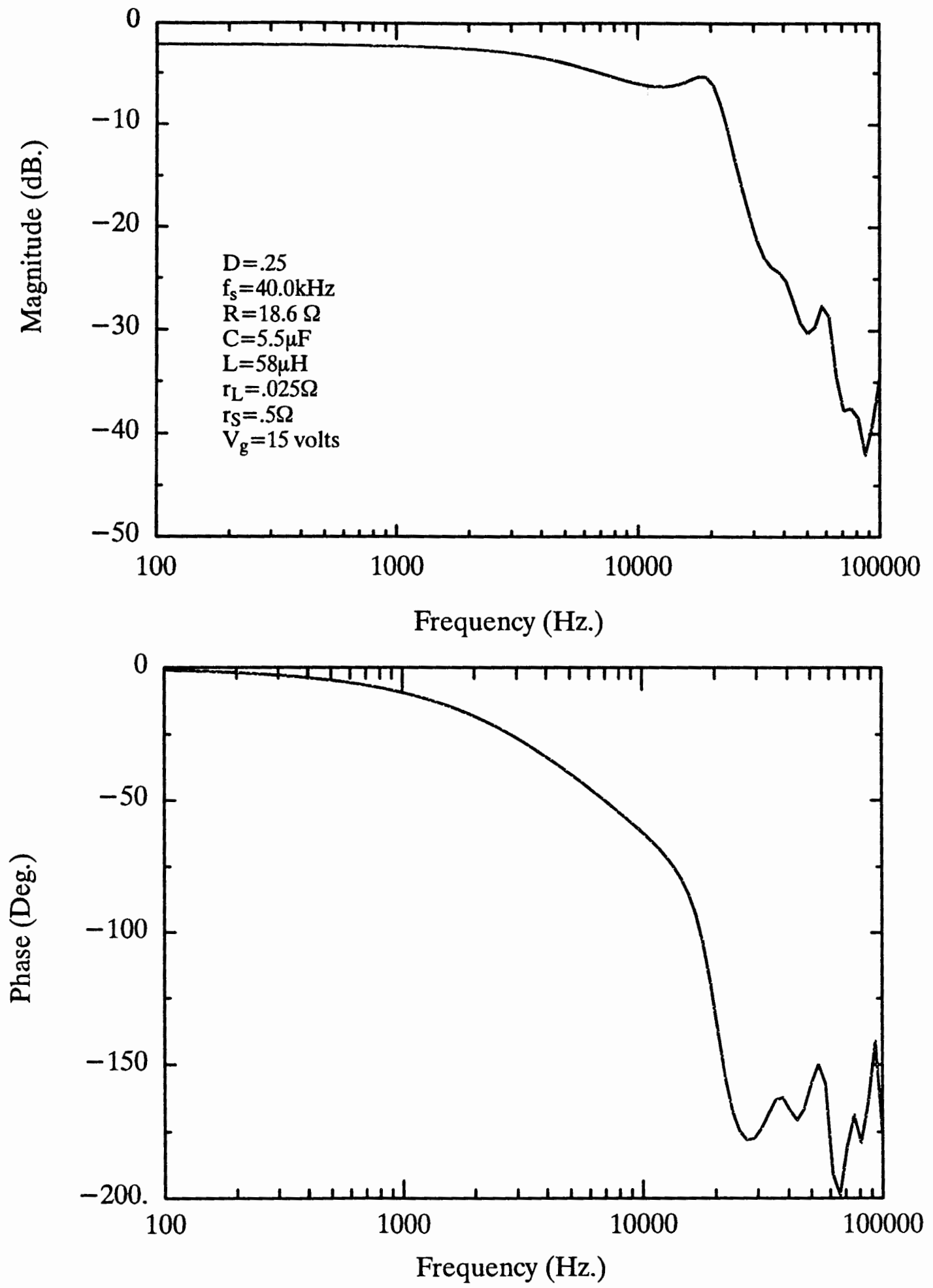

Figure $6-7$ The input-to-output response of the PWM boost converter operating in CCM under current mode programming ; exact (solid line) versus the third model (dashed line). 

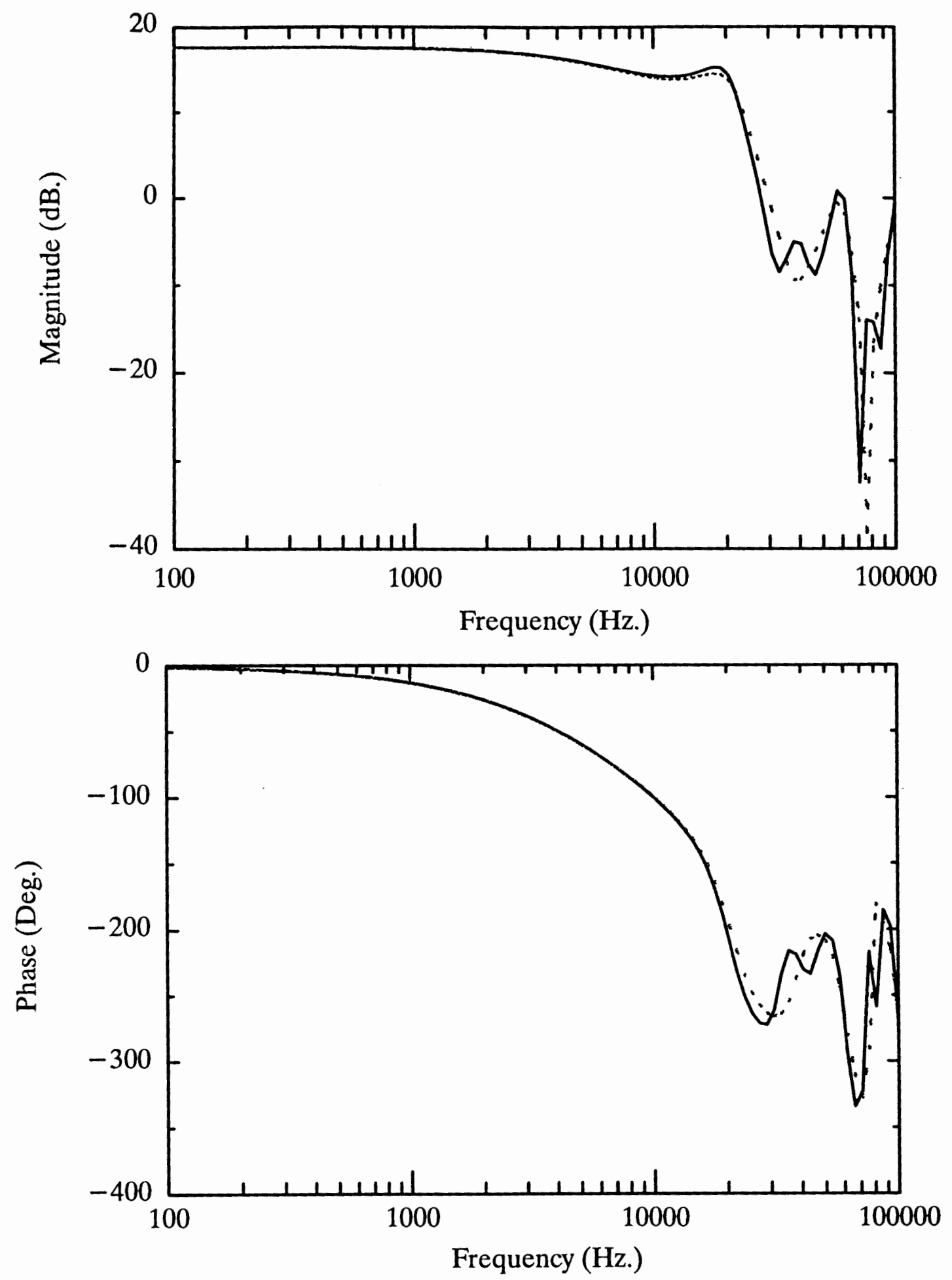

Figure 6-8 The control-to-output response of the PWM boost converter operating in $\mathrm{CCM}$ under current mode programming. Exact (solid line) versus the reduced order third model (dashed line). 

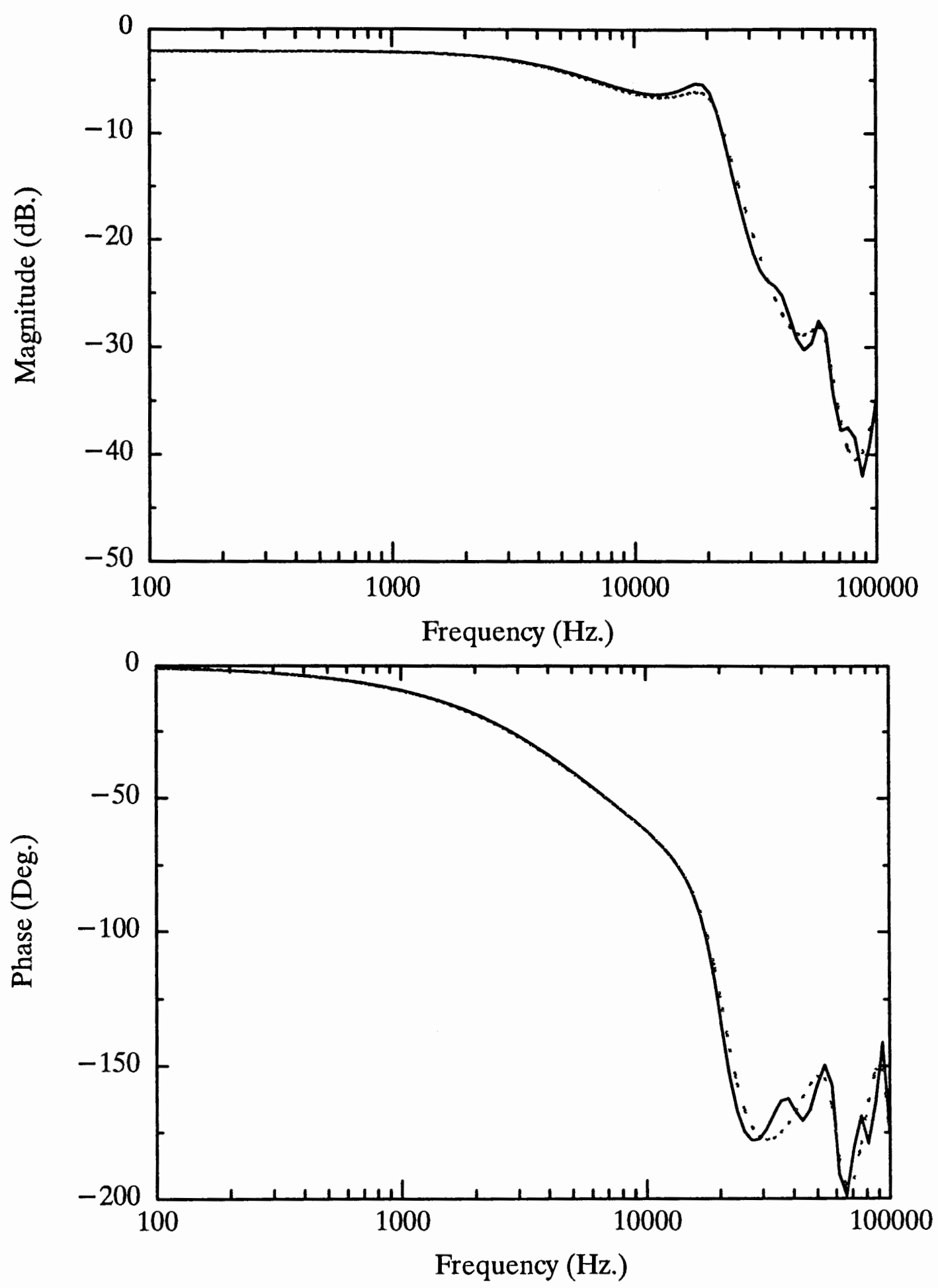

Figure 6 - 9 The input-to-output response of the PWM boost converter operating in CCM under current mode programming. Exact (solid line) versus the reduced order third model (dashed line). 
The expressions for the current mode parameters are obtained using Maple V. As mentioned before, the new parameters are lengthy. Whenever possible, they are reduced by employing some algebraic manipulations and simplifications. Again $v_{i d}, z_{i}$, $i_{o d}$, and $h_{f}$ parameters are obtained using the same technique as in the previous approach and are not repeated here. The following is a list of parameters that are different from the expressions obtained using the previous modeling technique:

$$
\begin{gathered}
h_{r}{ }^{\prime}=\frac{j \mu V_{p a} h^{T}}{T_{s} \omega L} \frac{e^{-j \omega D T_{s}}\left(1-e^{-j \omega D^{\prime} T_{s}}\right)}{\left(1-\Gamma e^{-j \omega T_{s}}\right)}+D^{\prime} \\
y_{o}{ }^{\prime}=\frac{\left(1-j \omega D^{\prime} T_{s}\right)\left(1+\Gamma e^{-j \omega T_{s}}\right)-(1+\Gamma) e^{-j \omega D^{\prime} T_{s}}}{\omega^{2} L T_{s}\left(1-\Gamma e^{-j \omega T_{s}}\right)} \\
-\frac{j h^{T} I_{c}}{\omega L T_{s}} \frac{\left(e^{-j \omega D T}-e^{-j \omega T_{s}}\right)}{\left(1-\Gamma e^{-j \omega T_{s}}\right)} \\
y_{r}=\frac{(\Gamma-1)\left(e^{-j \omega D T_{s}}-e^{-j \omega T_{s}}\right)}{\omega^{2} L T_{s}\left(1-\Gamma e^{-j \omega T_{s}}\right)}+j \frac{D^{\prime}}{\omega L}
\end{gathered}
$$

Then equation $(6-17)$ is used to find the new $h_{r}$ parameter:

$$
\begin{aligned}
h_{r}=\left(\frac{j \mu V_{p a} h^{T}}{T_{s} \omega L} \frac{e^{-j \omega D T_{s}}\left(1-e^{-j \omega D^{\prime} T_{s}}\right)}{\left(1-\Gamma e^{-j \omega T_{s}}\right)}+D^{\prime}\right) \\
-\left\{\left(\frac{-j \omega V_{p a} \mu h^{T}\left(1-e^{\left.-j \omega T_{s}\right)}\right.}{1-\Gamma-j \omega T_{s}-\left(1-\Gamma-j \omega T_{s} \Gamma\right) e^{-j \omega T_{s}}}\right) .\right. \\
\left.\left(\frac{(\Gamma-1)\left(e^{\left.-j \omega D T_{s}-e^{-j \omega T_{s}}\right)}\right.}{\omega^{2} L T_{s}\left(1-\Gamma e^{-j \omega T_{s}}\right)}+j \frac{D^{\prime}}{\omega L}\right)\right\}
\end{aligned}
$$

The $y_{o}$ parameter is found using equation $(6-18)$ 


$$
\begin{gathered}
y_{o}=\left(\frac{\left(1-j \omega D^{\prime} T_{s}\right)\left(1+\Gamma e^{-j \omega T_{s}}\right)-(1+\Gamma) e^{-j \omega D^{\prime} T_{s}}}{\omega^{2} L T_{s}\left(1-\Gamma e^{-j \omega T_{s}}\right)}-\frac{j h^{T_{I_{c}}}}{\omega L T_{s}} \frac{\left(e^{-j \omega D T_{s}}-e^{-j \omega T_{s}}\right)}{\left(1-\Gamma e^{-j \omega T_{s}}\right)}\right) \\
-\left\{\left(\frac{1-j \omega D^{\prime} T_{s}-e^{-j \omega D^{\prime} T_{s}}-\Gamma\left(1-e^{-j \omega D^{\prime} T_{s}}-j \omega D^{\prime} T_{s} e^{\left.-j \omega T_{s}\right)}\right.}{1-\Gamma-j \omega T_{s}-\left(1-\Gamma-j \omega T_{s} \Gamma\right) e^{-j \omega T_{s}}}\right.\right. \\
\left.+\frac{j \omega \mu h^{T} I_{c}\left(1-e^{-j \omega T_{s}}\right)}{1-\Gamma-j \omega T_{s}-\left(1-\Gamma-j \omega T_{s} \Gamma\right) e^{-j \omega T_{s}}}\right) \cdot \\
\left.\left(\frac{(1-\Gamma)\left(e^{-j \omega D T_{s}}-e^{-j \omega T_{s}}\right)}{\omega^{2} L T_{s}\left(1-\Gamma e^{-j \omega T_{s}}\right)}+j \frac{D^{\prime}}{\omega L}\right)\right\} \quad(6-31)
\end{gathered}
$$

Next equations $(5-3)$ and $(5-4)$ will be used to find the new $v_{i d}$ and $i_{o d}$ parameters.

$$
\begin{aligned}
& v_{i d}=-\frac{V_{p a}\left(1-e^{\left.-j \omega T_{s}\right)}\right.}{\left(1-\Gamma e^{\left.-j \omega T_{s}\right)}\right.}-\left\{\left(\frac{-j \omega V_{p a} \mu h^{T}\left(1-e^{-j \omega T_{s}}\right)}{1-\Gamma-j \omega T_{s}-\left(1-\Gamma-j \omega T_{s} \Gamma\right) e^{-j \omega T_{s}}}\right) .\right. \\
& \left.\left(-\frac{j V_{p a}}{\omega L} \frac{\left(1-e^{-j \omega T_{s}}\right)}{\left(1-\Gamma e^{-j \omega T_{s}}\right)}\right)\right\} \\
& i_{o d}=-\left(\frac{I_{c}\left(1-e^{-j \omega T_{s}}\right)}{\left(1-\Gamma e^{\left.-j \omega T_{s}\right)}\right.}+\frac{j V_{p a}\left(1-e^{-j \omega D^{\prime} T_{s}}\right)}{\omega L\left(1-\Gamma e^{\left.-j \omega T_{s}\right)}\right.}\right)- \\
& \left\{\left(\frac{1-j \omega D^{\prime} T_{s}-e^{-j \omega D^{\prime} T_{s}}-\Gamma\left(1-e^{-j \omega D^{\prime} T_{s}}-j \omega D^{\prime} T_{s} e^{-j \omega T_{s}}\right)}{1-\Gamma-j \omega T_{s}-\left(1-\Gamma-j \omega T_{s} \Gamma\right) e^{-j \omega T_{s}}}\right.\right. \\
& \left.+\frac{j \omega \mu h^{T} I_{c}\left(1-e^{-j \omega T_{s}}\right)}{1-\Gamma-j \omega T_{s}-\left(1-\Gamma-j \omega T_{s} \Gamma\right) e^{-j \omega T_{s}}}\right) . \\
& \left.\left(-\frac{j V_{p a}}{\omega L} \frac{\left(1-e^{-j \omega T_{s}}\right)}{\left(1-\Gamma e^{\left.-j \omega T_{s}\right)}\right.}\right)\right\}
\end{aligned}
$$

Equations $(6-30),(6-31),(6-32)$, and $(6-33)$ were given so one could get a feel for how complex the new parameters have become. For simulation purposes, 
their compact forms which are given by equations $(6-17),(6-18),(5-38)$, and $(5-39)$ will be used.

The new hybrid parameter switch model for the current mode programming could be represented by Figure $6-10$.

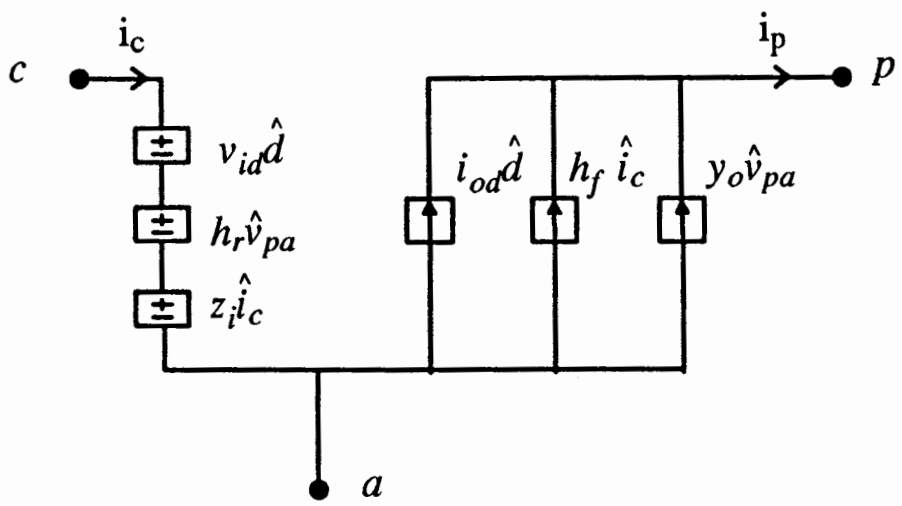

Figure 6 - 10 Hybrid parameter switch model for current mode programming.

It can be shown that the current programmed switch model can be used to analyze the PWM duty ratio programmed circuits. This is easily done by setting the $h^{T}$ of the current programmed model to zero. 


\subsection{Buck Converter Analysis}

In this section, the proposed hybrid parameter switch model is used to draw equivalent small signal model for the buck converter in a) duty ratio programmed; b) current programmed. Next, the accuracy of these models are examined by plotting the frequency responses of each model versus that of the exact results.

\subsubsection{PWM Duty Ratio Programmed Buck Converter}

The new hybrid parameter switch model can be used to construct an equivalent small signal circuit model for the buck converter of Figure $5-13$. This model is shown in Figure 6-11. The analysis of the new model is essentially the same as that of the second model. The only difference is the addition of the $v_{p a}$ dependent current source appearing as $y_{o} \hat{v}_{p a}$.

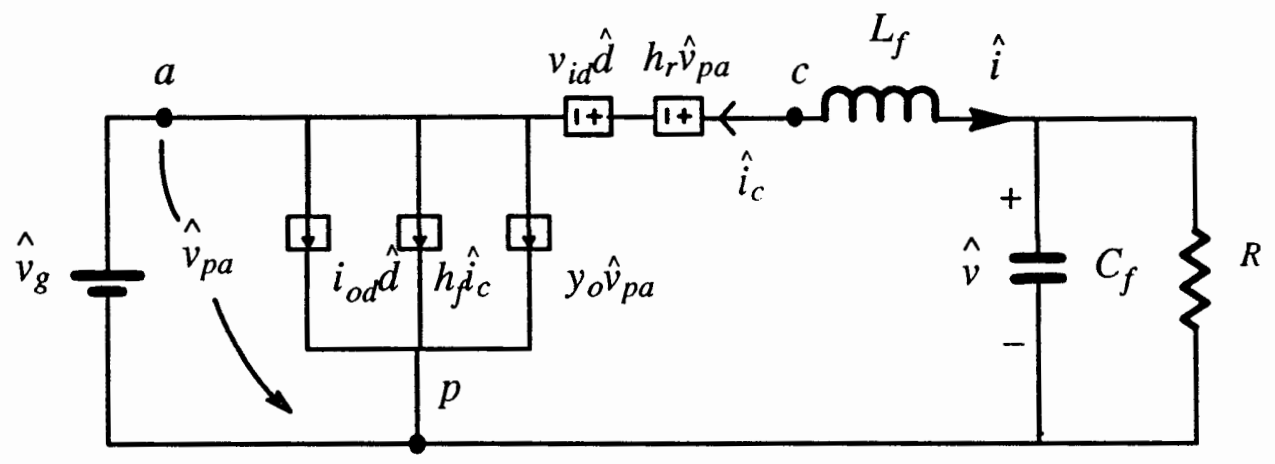

Figure 6 - 11 Hybrid parameter switch model for small-signal analysis of the buck converter of Figure $5-13$.

The state equation of Figure $6-11$ is found to be:

$\frac{d}{d t}\left[\begin{array}{l}\hat{i} \\ \hat{v}\end{array}\right]=\left[\begin{array}{cc}0 & -\frac{1}{L_{f}} \\ \frac{1}{C_{f}} & -\frac{1}{R C_{f}}\end{array}\right]\left[\begin{array}{c}\hat{i} \\ \hat{v}\end{array}\right]+\left[\begin{array}{c}\frac{1-h_{r}}{L_{f}} \\ 0\end{array}\right] \hat{v}_{g}+\left[\begin{array}{c}\frac{v_{i d}}{L_{f}} \\ 0\end{array}\right] \hat{d}$ 
The control-to-output and input-to-output frequency responses of the hybrid model for the buck converter are plotted against the exact results as shown in Figure $6-12$ and Figure $6-13$, respectively. There are no discrepancies in the results over the an entire range of frequencies. 

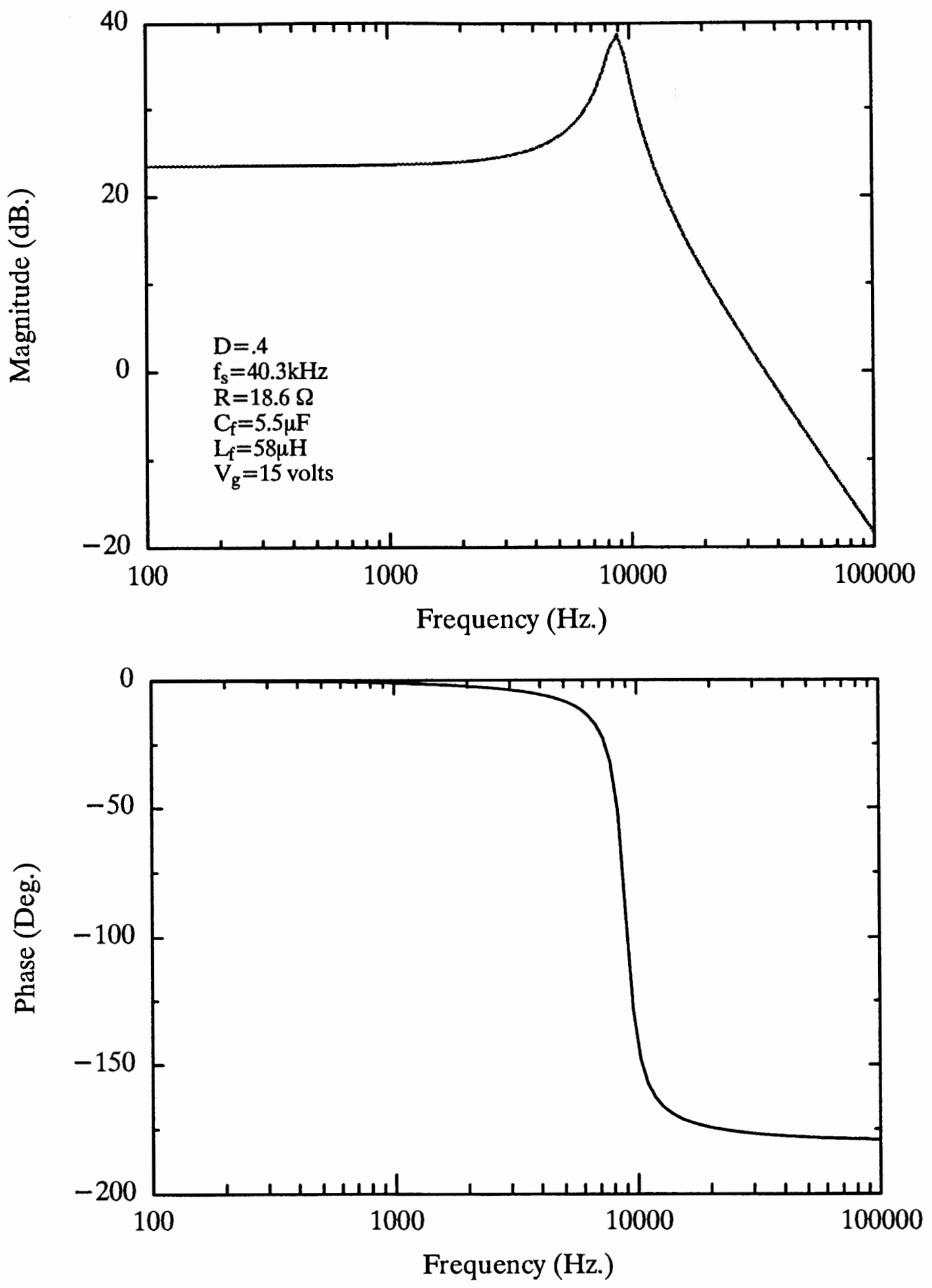

Figure 6-12 The control-to-output response of the PWM buck converter operating in CCM; exact (solid line) versus the third model (dashed line). 

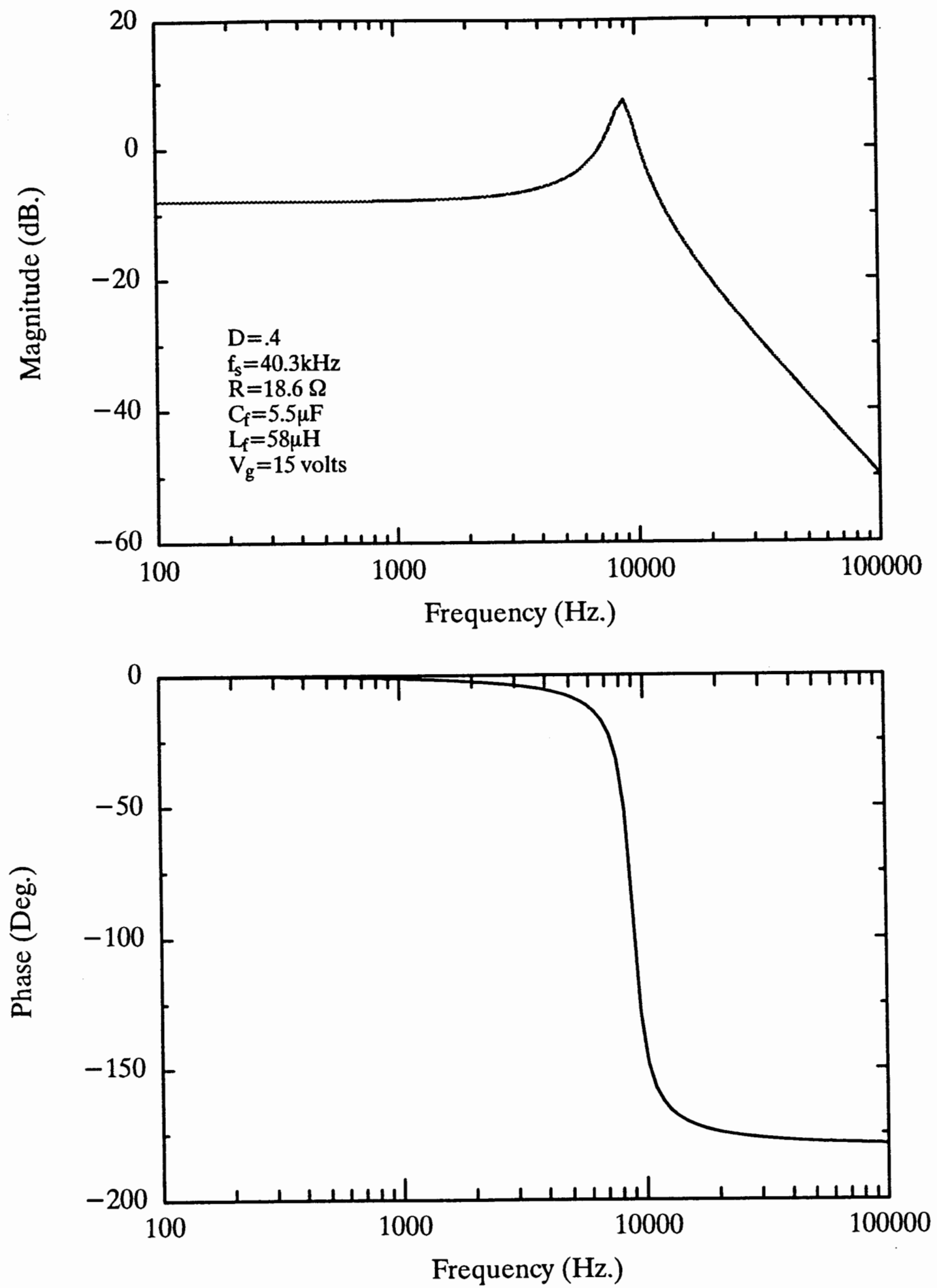

Figure 6- 13 The input-to-output response of the PWM buck converter operating in CCM ; exact (solid line) versus the third model (dashed line). 


\subsubsection{PWM Current Programmed Buck Converter}

Figure $6-14$ shows the equivalent hybrid model for the buck converter under current mode control.

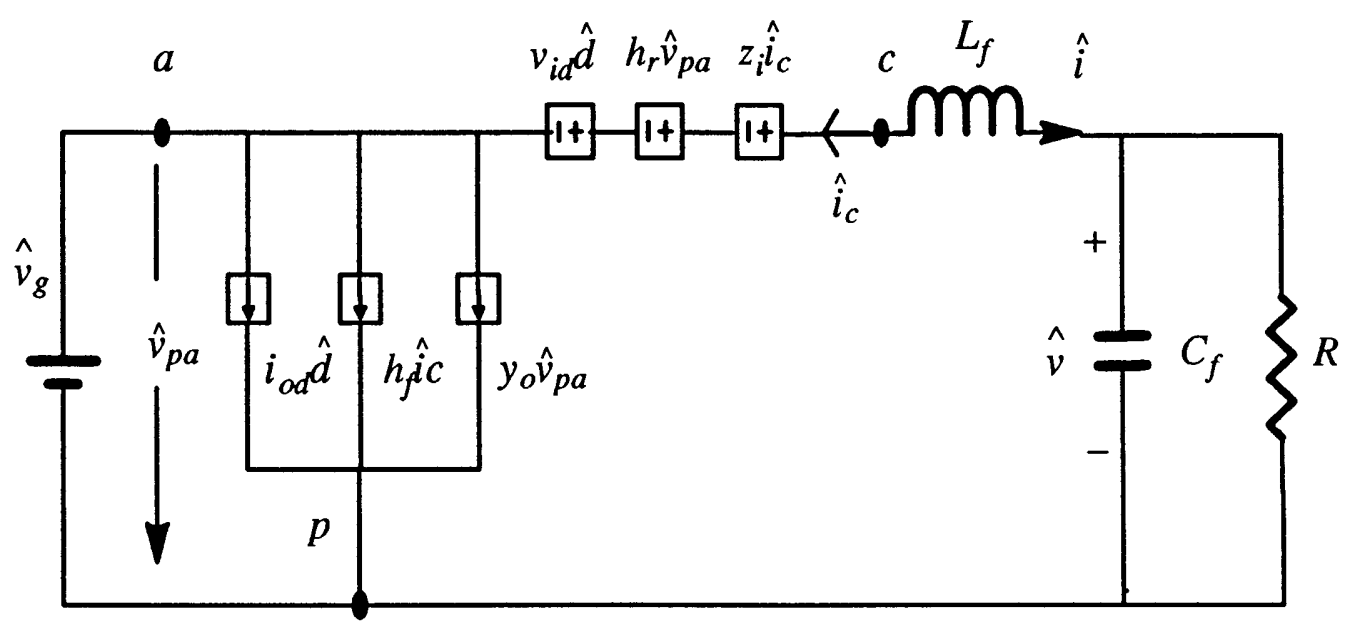

Figure 6 - 14 Hybrid parameter switch model for small-signal analysis of the Current programmed buck converter of Figure 5-19.

The small signal state equation of the above current programmed model is:

$\frac{d}{d t}\left[\begin{array}{l}\hat{i} \\ \hat{v}\end{array}\right]=\left[\begin{array}{cc}-\frac{z_{i}}{L_{f}} & -\frac{1}{L_{f}} \\ \frac{1}{C_{f}} & -\frac{1}{R C_{f}}\end{array}\right]\left[\begin{array}{l}\hat{i} \\ \hat{v}\end{array}\right]+\left[\begin{array}{c}\frac{1-h_{r}}{L_{f}} \\ 0\end{array}\right] \hat{v}_{g}+\left[\begin{array}{c}v_{i d} \\ L_{f} \\ 0\end{array}\right] \hat{d}$

Using equation $(6-35)$, the input-to-output and control-to-output transfer functions can be found. Next, the magnitude and phase plots of these transfer functions are compared against the exact model plots.

As shown in Figure 6 - 15, the control-to-output magnitude and phase plots of the new model accurately predict the exact small-signal results except at the switching frequency $f_{s}$ and its first harmonic. The input-to-output frequency response of the model is exact as shown in Figure $6-16$. This is an excellent improvement over the previous model. 

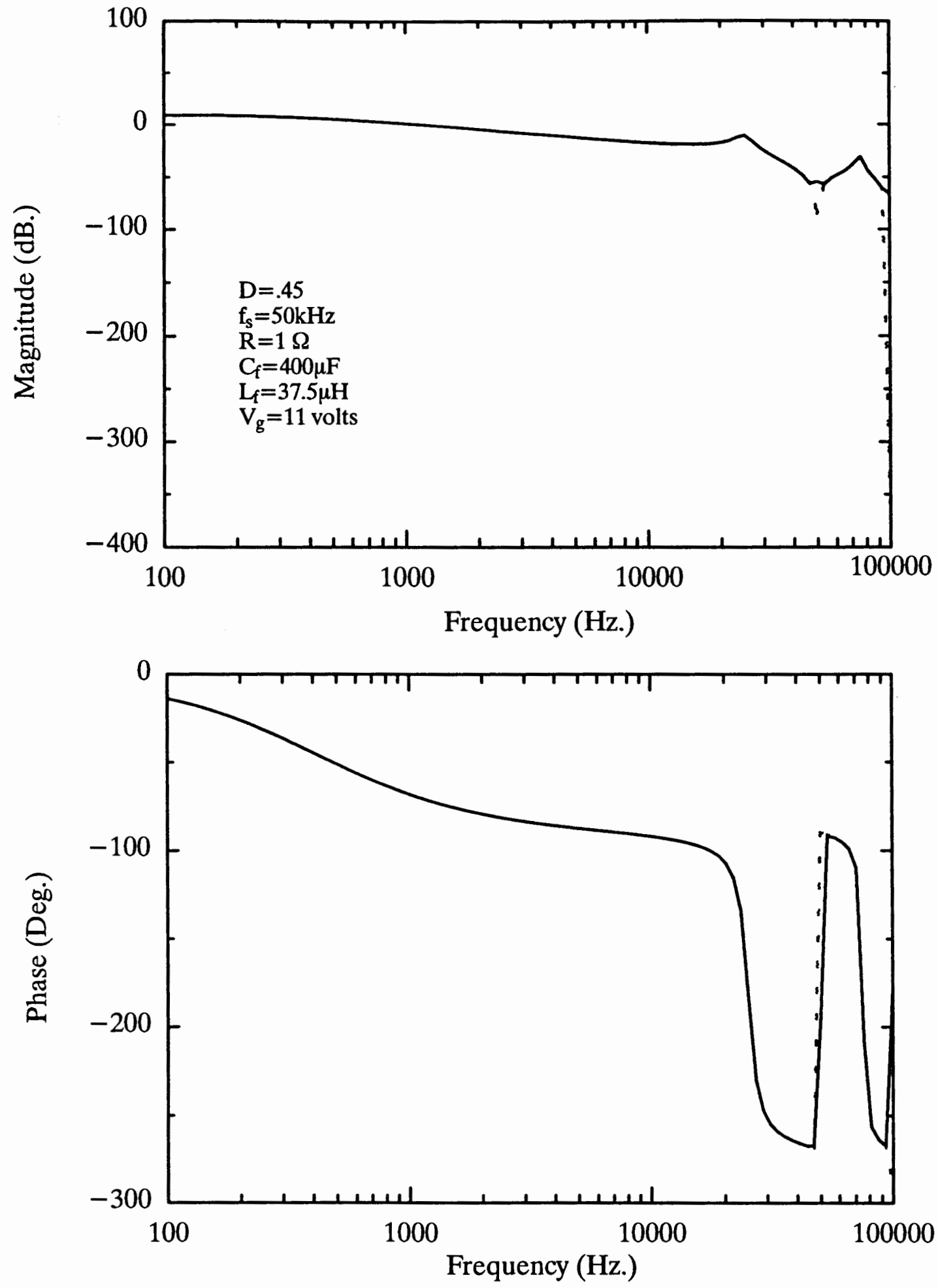

Figure 6 - 15 The control to output response of the PWM buck converter operating in CCM under current mode programming; exact (solid line) versus the third model (dashed line). 

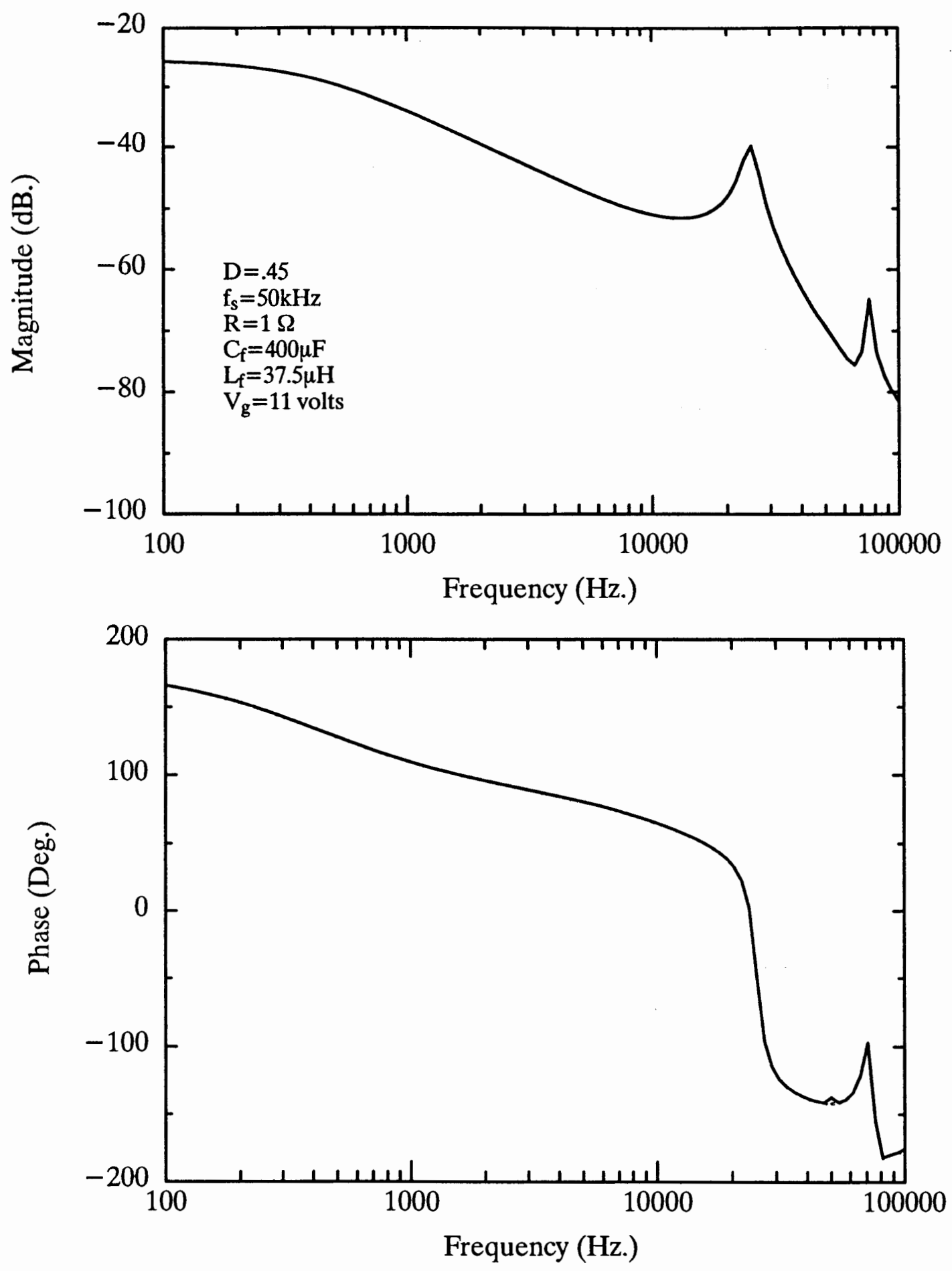

Figure 6-16 The input-to-output response of the PWM buck converter operating in CCM under current mode programming; exact (solid line) versus the third model (dashed line). 


\subsection{Buckboost Converter Analysis}

In this section, the new hybrid parameter switch model is used to perform small signal analysis of a basic buckboost converter. The objective is to examine the accuracy of the model. Next, the frequency response of the equivalent buckboost model is compared with that of the exact method.

\subsubsection{PWM Duty Ratio Programmed Buckboost Converter}

The equivalent small signal model of the buckboost converter of Figure $5-23$ is shown in Figure $6-17$. The state equation of this model can be found using analysis presented in section5.3.1.

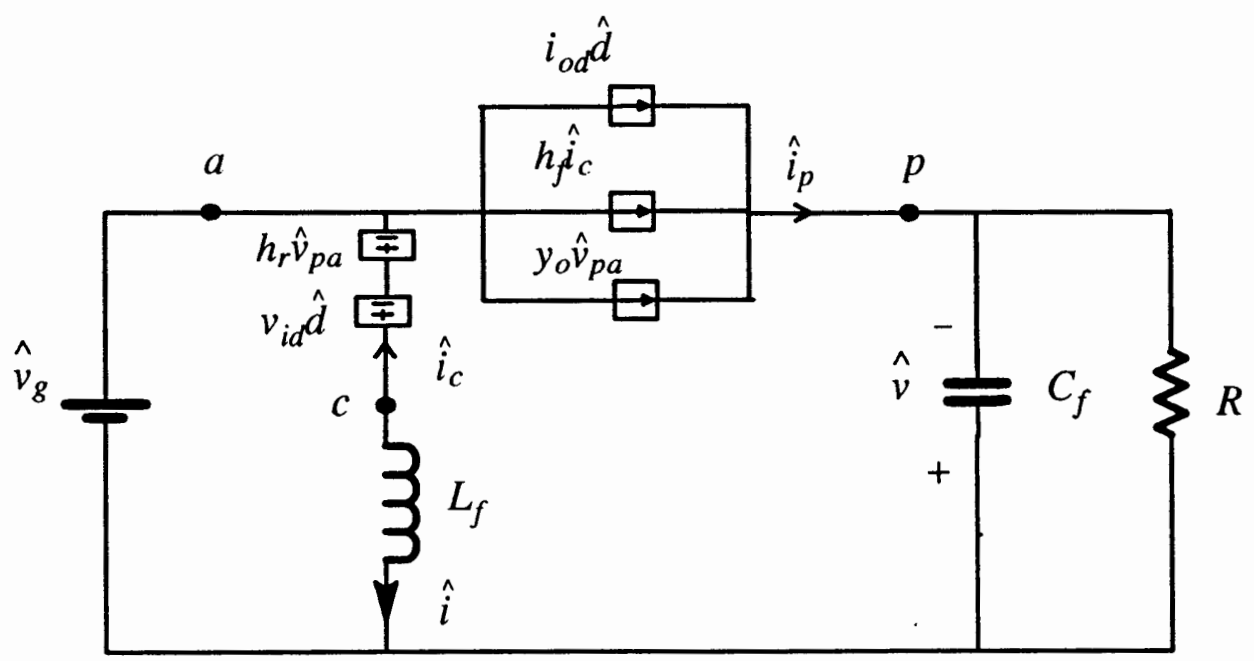

Figure 6 - 17 Small-signal hybrid parameter switch model for the PWM duty-ratio buck-boost converter of Figure 5-23.

The state equation of Figure $6-17$ is:

$\frac{d}{d t}\left[\begin{array}{c}\hat{i} \\ \hat{v}\end{array}\right]=\left[\begin{array}{cc}0 & -\frac{h_{r}}{L_{f}} \\ \frac{h_{f}}{C_{f}} & \frac{y_{o}-\frac{1}{R}}{C_{f}}\end{array}\right]\left[\begin{array}{c}\hat{i} \\ \hat{v}\end{array}\right]+\left[\begin{array}{c}\frac{1-h_{r}}{L_{f}} \\ \frac{y_{o}}{C_{f}}\end{array}\right] \hat{v}_{g}+\left[\begin{array}{c}\frac{v_{i d}}{L_{f}} \\ -\frac{i_{o d}}{C_{f}}\end{array}\right] \hat{d}$ 
As shown in Figure 6-18, the control-to-output frequency response of the new model matches that of the exact results. The only difference appears to be at the switching frequency, $f_{s}$.

The input-to-output magnitude and phase responses of the model are plotted in Figure 6 - 19. The exact input-to-output results are also plotted on the same graph. The magnitude agreement is excellent. The phase response of the model fails to predict exact results beyond the switching frequency, $f_{s}$. 

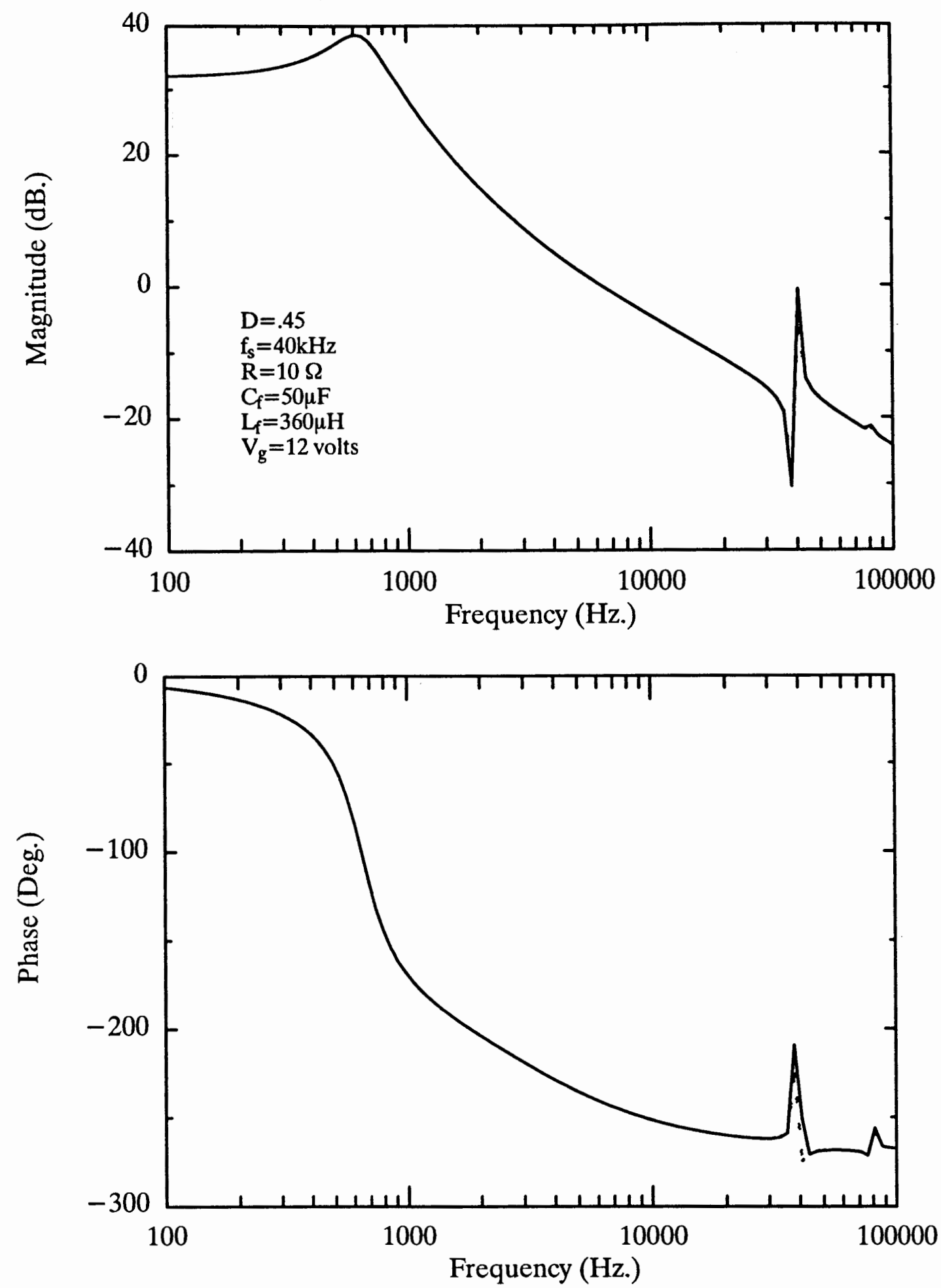

Figure 6-18 The control-to-output response of the PWM buckboost converter operating in CCM; exact (solid line) versus the third model (dashed line). 

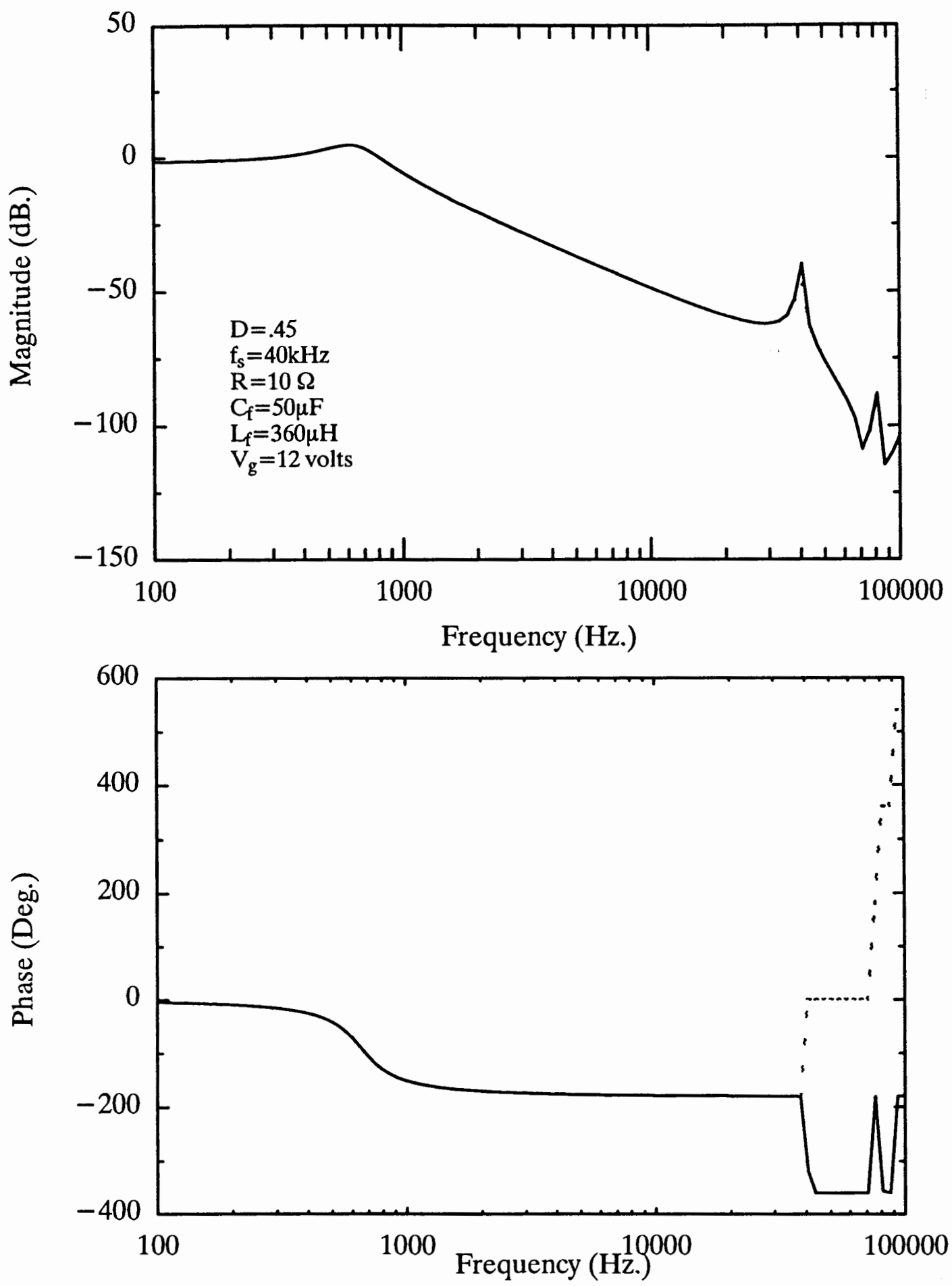

Figure 6-19 The input-to-output response of the PWM buck-boost converter operating in CCM ; exact (solid line) versus the third model (dashed line). 


\subsubsection{PWM Current Programmed Buckboost Converter}

The state equation for the current mode buckboost model shown in Figure $6-20$ is given below:

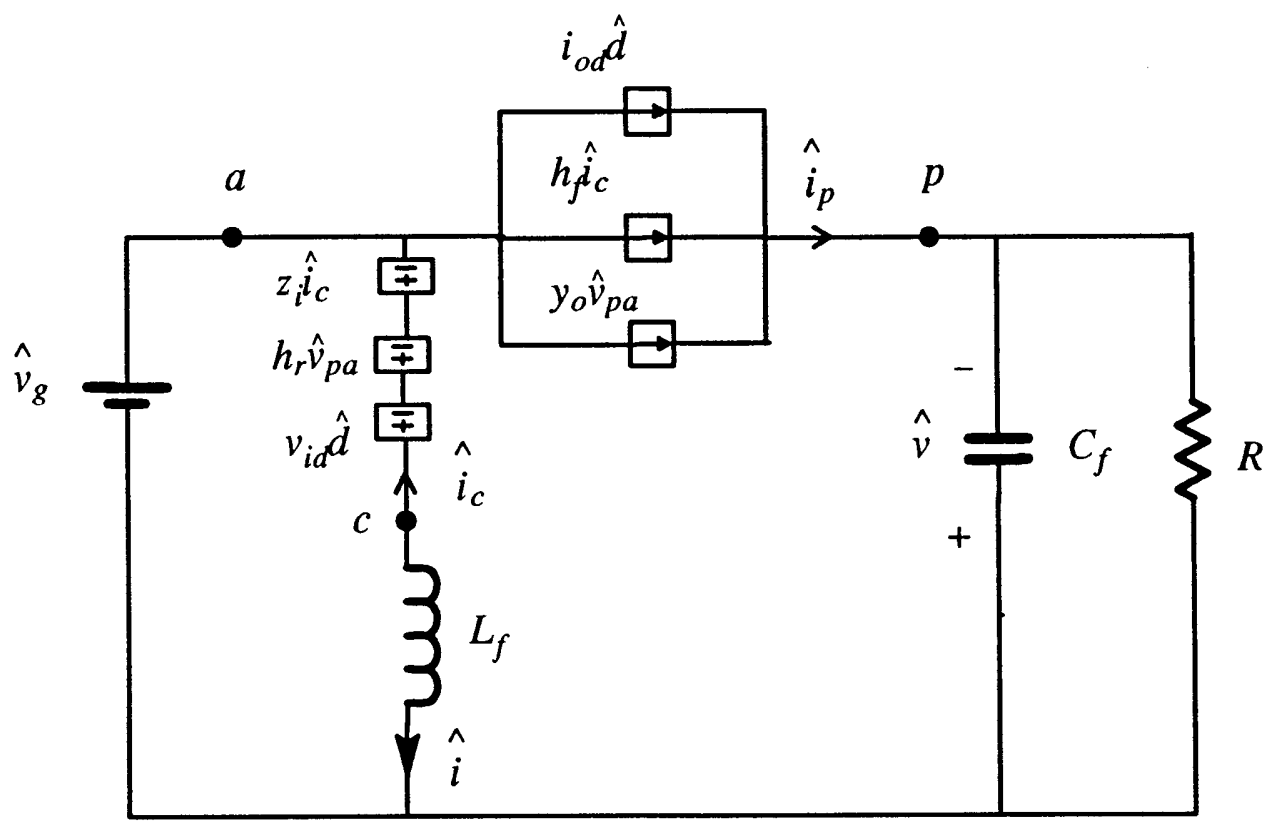

Figure 6-20 Small-signal equivalent model for current mode analysis of the buckboost converter in Figure 5 - 23.

$\frac{d}{d t}\left[\begin{array}{l}\hat{i} \\ \hat{v}\end{array}\right]=\left[\begin{array}{cc}-\frac{z_{i}}{L_{f}} & -\frac{h_{r}}{L_{f}} \\ \frac{h_{f}}{C_{f}} & \frac{y_{o}-\frac{1}{R}}{C_{f}}\end{array}\right]\left[\begin{array}{l}\hat{i} \\ \hat{v}\end{array}\right]+\left[\begin{array}{c}\frac{1-h_{r}}{L_{f}} \\ \frac{y_{o}}{C_{f}}\end{array}\right] \hat{v}_{g}+\left[\begin{array}{c}\frac{v_{i d}}{L_{f}} \\ -\frac{i_{o d}}{C_{f}}\end{array}\right] \hat{d}$

As shown in Figure $6-21$, the control to output responses of the model are exact up to at least twice the switching frequency. The input-to-output magnitude and phase responses of this model are shown in Figure $6-22$. They are also exact up to twice the switching frequency. 

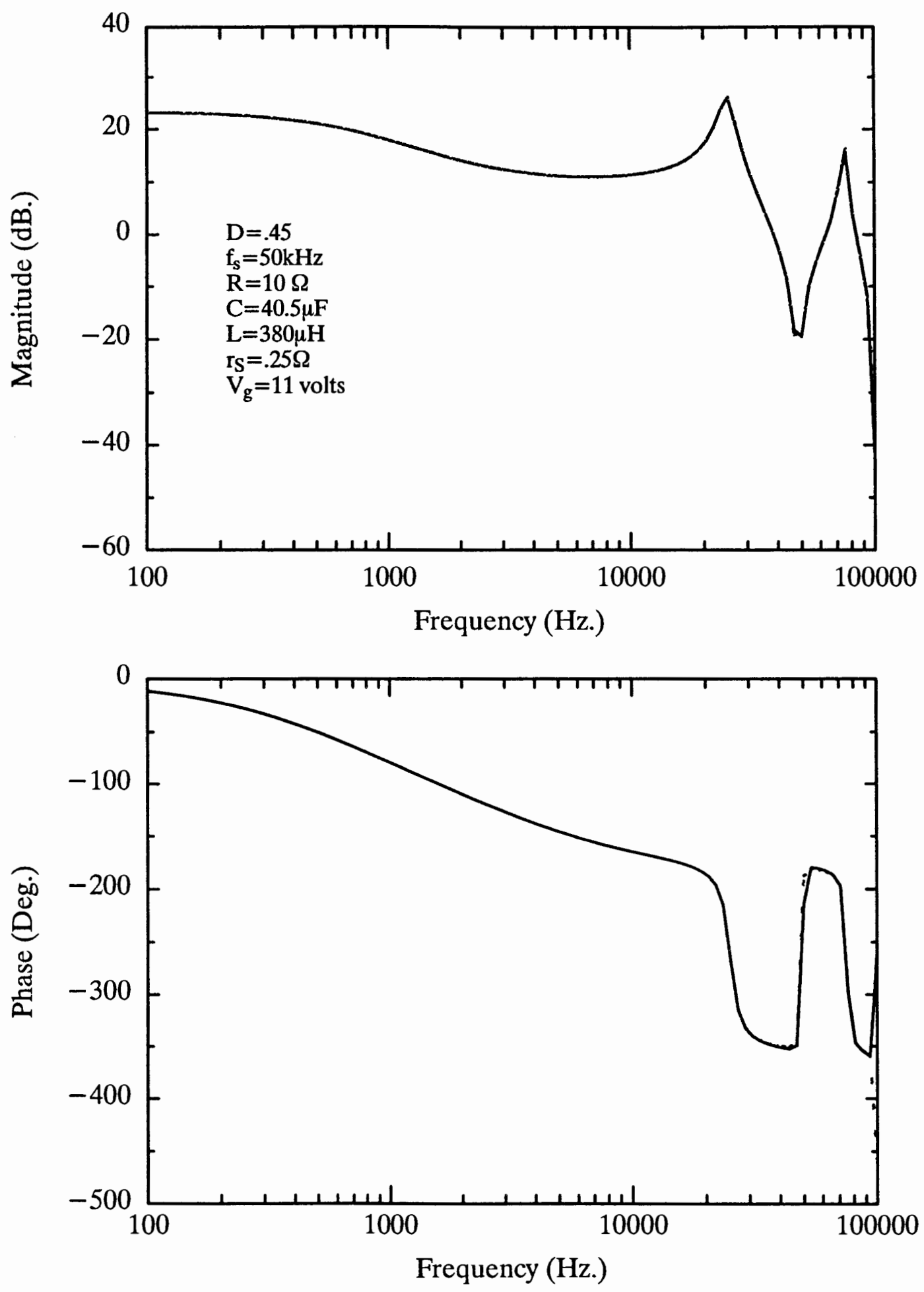

Figure 6-21 The control-to-output response of the PWM buckboost converter operating in CCM under current mode programming; exact (solid line) versus the third model (dashed line). 

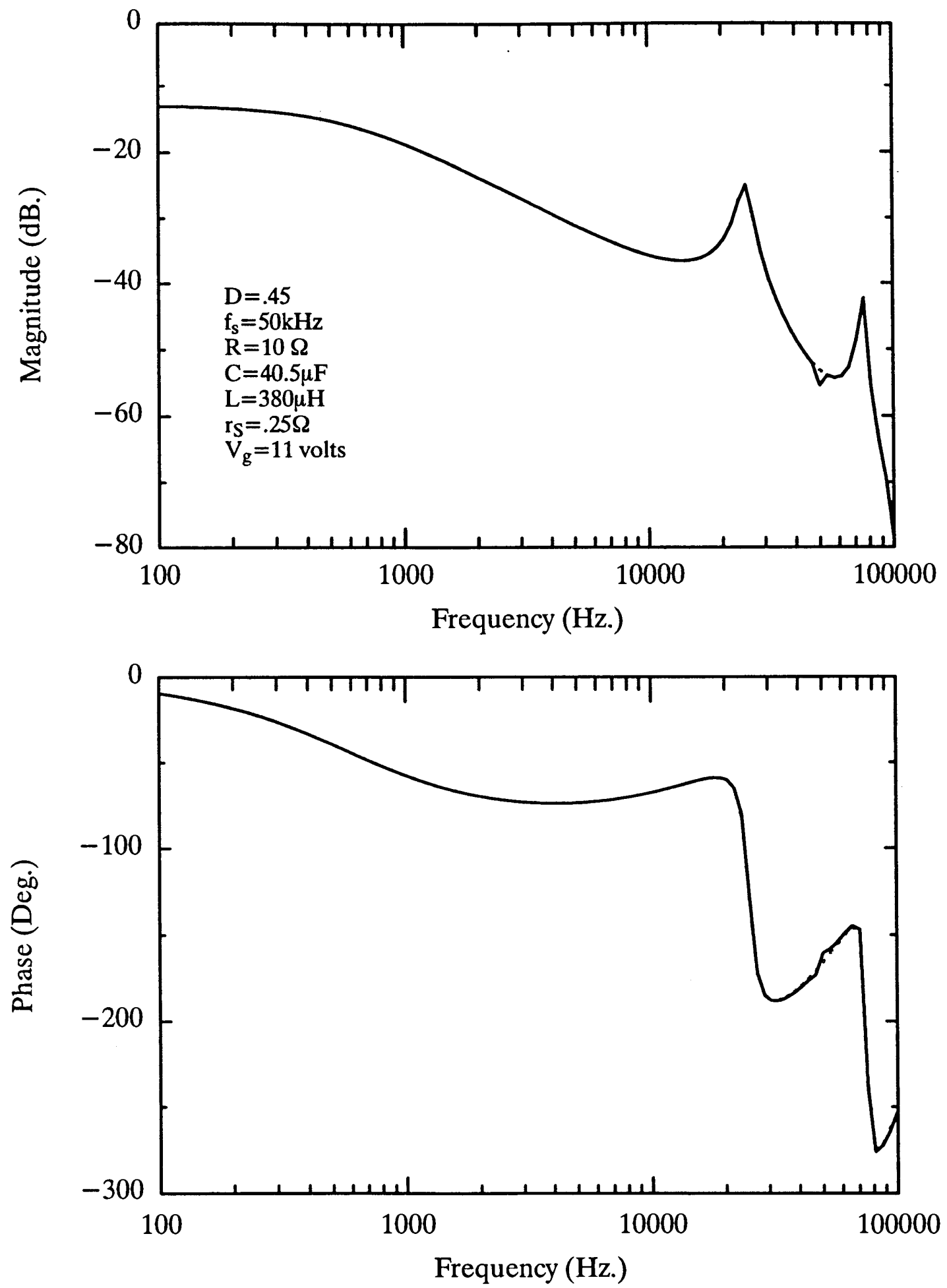

Figure 6-22 The input-to-output response of the PWM buckboost converter operating in CCM under current mode programming; exact (solid line) versus the third model (dashed line). 


\title{
CHAPTER 7 CONCLUSION
}

\begin{abstract}
A new theory in modeling the power converters was presented and a unified small signal switch model was obtained. The aim of this thesis was originally to derive a simple model that could be used for both circuit and simulation analysis. The new model was found through modeling the PWM switch as a hybrid parameter two-port network. In this modeling approach, exact small-signal transfer functions [1]-[3] for two cases were considered and consequently two models were found: 1) PWM dutyratio converters in CCM, 2) PWM current mode control converters in CCM. The new method was considered to be general and applicable to any converter circuit. Using this method other models besides the switch model can be developed. In chapter 4 , the first modeling approach was presented. This model failed to produce accurate controlto-output response because when both inputs (states) were nulled, the control-tooutput equation reduced to the state space averaging model. A second (new) method was presented in chapter 5 to overcome the inaccuracies of the previous method. The control-to-output and input-to-output frequency responses of the second model were plotted with the exact small-signal responses and showed to be exact for the full order system. Therefore, the next step was to obtain expressions for the parameters. In order to reduce the complexity of the expressions, the output capacitor was replaced with an independent voltage source. Then, using this second model, equivalent smallsignal models for buck and buckboost converters were found. From these models, the
\end{abstract}


state space representation of the buck and buckboost converters were derived. Then, the responses were plotted with the responses obtained through the exact small-signal analysis. The PWM duty-ratio switch model results were satisfactory. The PWM current programmed model did not produce accurate input-to-output results. As explained in chapter 6 , when the full order state equations were reduced to a single state, the effect of current sensing disappeared from the equations, hence; the current mode model was very inaccurate. In chapter 6 , the third method was presented which successfully worked for both control schemes in CCM. This model (third model) is very accurate up to the switching frequency. The third model was applied to the buck and buckboost converters and the frequency response results were excellent. It should be noted here that some of the current mode expressions for the parameters were lengthy. This model can be easily coded for simulations.

There are significant advantages in using this modeling approach over previous current mode or duty ratio models:

- This modeling approach can be applied to any three terminal component of the converter, i.e. If needed one can use it to model the converter as a y-parameter model, etc.

- The (third) switch model applies to both control schemes, i.e. setting $h^{T}=0$ reduces the current mode model to duty ratio model.

- The results can be evaluated using state space transfer functions. Therefore, different transfer functions can be tested. Stability analysis is straightforward, i.e. location of poles and zeros can be determined using the state space analysis. 
The disadvantages are that the switch parameters are lengthy at this time and cannot be used for circuit analysis by hand. It should also be noted that the lengthy expressions are found for the switch model, and one can try to use the new theory (two-port analysis) on the whole circuit and see how lengthy the expressions will be.

The switch modeling approach can be extended to Discontinuous Conduction Mode (DCM). At this time, these models are expected to be lengthy in derivations. Once a method is found to reduce the parameter expressions, the DCM model will also be developed. 


\section{REFERENCES}

[1] R. Tymerski, "Application of the Time Varying Transfer Function for Exact Small-Signal Analysis," IEEE Power Electronics Specialists Conference (PESC) ‘91, June 1991, pp. 80-87.

[ 2] R. Tymerski, "Frequency Analysis of Time-Interval-Modulated Switched Networks," IEEE Transactions on Power Eelctronics, Vol. 6, No. 2, April 1991, pp. $287-295$.

[ 3 ] Weihe Niu. "The Exact Modeling of Time-Interval-Modulated Switched Networks," M.S. Thesis, Portland State University, Portland, Oregon, February 1993.

[4] B. Y. B. Lau and R. D. Middlebrook, "Small-Signal Frequency Response Theory for Piecewise-Constant Two-Switched-Network Dc-to-Dc Converters," IEEE Power Electronics Specialists Conference (PESC), 1986, pp. 186-200.

[ 5] R. D. Middlebrook and S. Cuk, "A General Unified Approach to Modelling Switching Converter Power Stages," IEEE Power Electronics Specialists Conference (PESC), 1976, pp. 18-31.

[ 6] Andrez Pietkiewicz and Daniel Tollik, "Unified Topological Modeling Method of Switching DC-DC Converters in Duty-Ratio Programmed Mode," IEEE Transactions on Power Electronics, Vol. PE-2, No. 3, July 1987, pp. 218-226.

[7] R. Tymerski and V. Vorpérian, "Generation, Classification and Analysis of Switched-Mode DC-to-DC Converters by the Use of Converter Cells," IEEE International Telecommunications Energy Conferece (INTELEC), October 1986, pp. 181-195. 
[ 8 ] R. Tymerski, V. Vorpérian, F. C. Lee, and W. T. Baumann, "Nonlinear Modeling of the PWM Switch," IEEE Transactions on Power Electronics, Vol. 4, No. 2, April 1989, pp. 225-234.

[ 9 ] V. Vorpérian, R. Tymerski, and F. C. Lee, "Equivalent Circuit Model for Resonant and PWM Switches," IEEE Transaction on Power Electronics, Vol. 4. No. 2 , April 1989, pp. 205-214.

[ 10 ] V. Vorpérian, "Simplified Analysis of PWM Converters Using Model of the PWM Switch: Part I and II," IEEE Transaction on Aerospace and Electronics Syst., Vol. 26, No. 2, May 1990.

[11] G. C. Verghese, C. A. Bruzos, and K. N. Mahabir, "Averaged and SampledData Models for Curren Mode Control: A Reexamination," IEEE Power Electronics Specialists (PECS), 1989, pp. 484-491.

[ 12] R. Tymerski and Duwang Li, "State-Space Models for Current Programmed Pulsewidth-Modulated Converters," IEEE Transactions on Power Electronics, Vol. 8, No. 3, July 1993, pp. 271-278.

[ 13] R. D. Middlebrook, “Topics in Multiple-Loop Regulators and Current Mode Programming," IEEE Power Electronics Specialists, 1985, pp. 716-732.

[ 14 ] S. Hsu, A. R. Brown, L. Resnick, and R. D. Middlebrook, "Modeling and Analysis of Switching DC-to-DC Converters in Constant-Frequency Current Programmed Mode," IEEE Power Electronics Specialists (PESC), 1979, pp. 284-301.

[ 15] V. Vorpérian, "Analysis of Current-Mode Controlled PWM Converters using the model of the current-controlled PWM Switch," Presented at Power Conversion and Intelligent Motion Conference, PCIM '90, Oct. 1990.

[ 16] R. Ridley, "A New Continuous-Time Model for Current Mode Control," IEEE Transaction on Power Electronics, Vol. 6, Apr. 1991, pp. 271-280. 